Historic, archived document

Do not assume content reflects current scientific knowledge, policies, or practices. 

Forest Service

Pacific Northwest

Research Station

Seasonal Composition

Research Paper

PNW-RP-382

[4iss

\section{of Invertebrates in}

Several Oregon

Streams

Pamela E. Porter and William R. Meehan

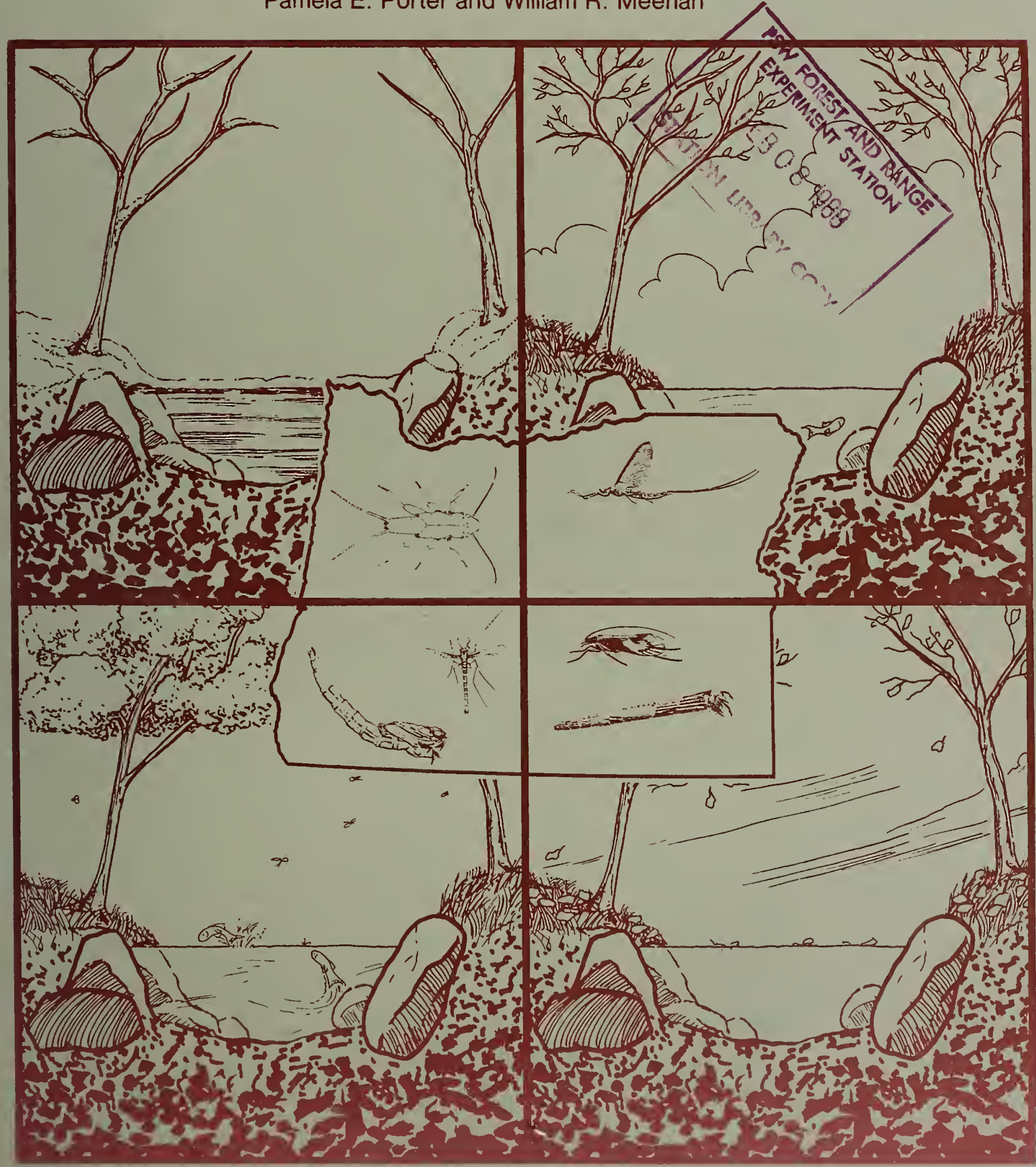


Abstract

Summary
Porter, Pamela E.; Meehan, William R. Seasonal species composition of invertebrates in several Oregon streams. Res. Pap. PNW-RP-382. Portland, OR: U.S. Department of Agriculture, Forest Service, Pacific Northwest Research Station; 1987. 36 p.

The invertebrate communities of eight Oregon streams were sampled seasonally from 1974 to 1976 . Benthic, drift, and two types of aerial-trap samples were collected. Occurrence and percentage composition are summarized by sample type, season, and geographic area (coastal, Cascade, central, and eastern Oregon). Within 276 families, 426 taxa were identified; the 20 families most prevalent within each sample type accounted for the majority of organisms collected (77.8-91.8 percent). In most areas and seasons, Diptera and Ephemeroptera together comprised over half of the invertebrates collected.

Keywords: Invertebrata, aquatic life, Oregon.

During 1974-76, we studied the community structure of invertebrates in streams in the coastal, Cascade, central, and eastern areas of Oregon. Benthic, drift, sticky-trap, and water-trap samples were collected seasonally. Occurrence and percentage composition of invertebrates were summarized by sample type, season, and geographic area.

When data for all sample types were combined, Diptera was the most abundant order collected in summer and fall, and Ephemeroptera were prevalent in winter. In most areas and seasons, combined numbers of Diptera and Ephemeroptera comprised over half of the invertebrates collected.

Within the benthic community, Ephemeroptera and Diptera were the most prevalent orders, although other orders such as Coleoptera and Plecoptera occasionally were quite abundant. Ephemeroptera, Diptera, Plecoptera, and Trichoptera were the dominant orders collected in drift samples. Diptera was the most prevalent order collected in sticky-trap and water-trap samples, except for occasional large numbers of Collembola.

Within 276 families, 426 taxa were collected during the study. When data were summarized by family, the 20 most prevalent families accounted for the majority of organisms collected (77.8-91.8 percent). 


\section{Contents}

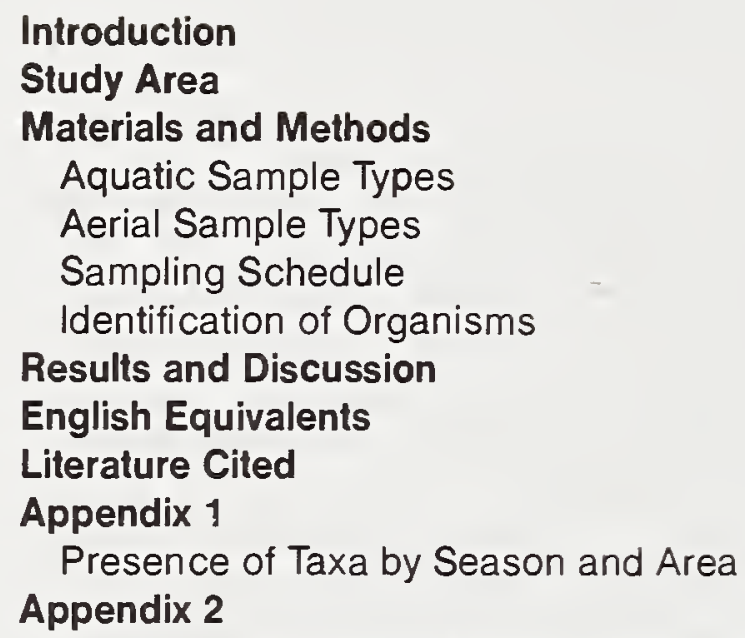

Percentage of Samples From Coastal Streams Containing Listed Taxa, by Season and Sample Type

22 Percentage of Samples From Cascade Streams Containing Listed Taxa, by Season and Sample Type

26 Percentage of Samples From Central Streams Containing Listed Taxa, by Season and Sample Type Percentage of Samples From Eastern Streams Containing Listed Taxa, by Season and Sample Type

\section{Appendix 3}

33 Summary of Invertebrate Data for Each Season and Sample Type-Coastal Streams

34 Summary of Invertebrate Data for Each Season and Sample Type-Cascade Streams

35 Summary of Invertebrate Data for Each Season and Sample Type-Central Streams

36 Summary of Invertebrate Data for Each Season and Sample Type-Eastern Streams 


\section{Introduction}

\section{Study Area}

During 1974-76, we studied the relation of riparian vegetation canopy to the community structure of invertebrates in several streams in Oregon. The composition of invertebrates occurring seasonally in the study streams was evaluated according to several sample types.

The purpose of this report is to provide a list of the invertebrate taxa found in the distinct geographic areas of Oregon in each season. This list will serve as a checklist for other studies of stream ecology in Oregon, and it will be the base of reference for future reports on effects of riparian canopy and food habits.

Eight streams in Oregon were selected for study (fig. 1)-two in each of four geographical areas: coastal Oregon, the west side of the Cascade Range, central Oregon, and eastern Oregon. Thus, a general transect of the State from west to east was sampled. All the study streams were second- or third-order streams, comparable in size, and representative of the small streams that furnish a large amount of rearing habitat for young salmon and trout. The study streams, by area, were:

Coastal (tributaries of Five Rivers in the Alsea River drainage):

\section{Green River}

East Fork Green River

Cascades (tributaries of the Lookout Creek system, which drains into the McKenzie

River):

Mack Creek

MacRae Creek

Central (tributaries of the Deschutes River drainage):

Ochoco Creek

Canyon Creek

\section{Eastern (tributaries of the Grand Ronde River): Meadow Creek McCoy Creek}

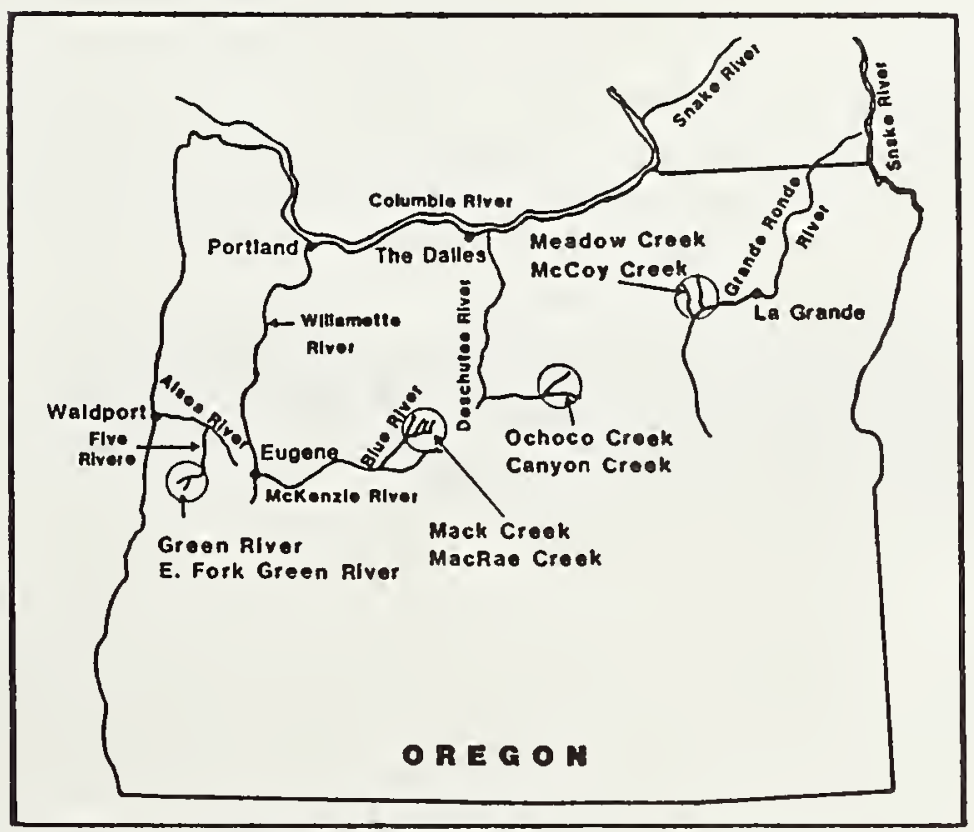

Figure 1-Locations of study streams. 


\section{Materials and Methods}

\author{
Aquatic Sample Types
}

In each of the eight streams, two similar reaches were sampled. Streamside vegetation was a mix of conifers, hardwoods, brush, and grass. Stream substrates were generally similar and ranged from cobble to coarse sand.

Benthic-Benthic samples were collected with a modified Hess sampler $1 /$ covering a surface area of $0.09 \mathrm{~m}^{2}$. Two samples were collected from each study reach at the beginning of a 16-day study period and again at the end of the 16 days; the samples were preserved in formal alcohol (half 70-percent ethanol and half 10-percent formalin). In the laboratory, invertebrate organisms were sorted from the samples, counted, and identified to the lowest possible taxonomic level (generally to family and, where feasible, to genus or species). After the invertebrates were sorted, the entire sample was freeze-dried and weighed on an analytical balance to the nearest $0.0001 \mathrm{~g}$.

Drift-Aquatic drift was sampled in each study section with a 280-micrometer mesh Nitex drift net, $76 \mathrm{~cm}$ long. Openings in the nets were 0.46 by $0.31 \mathrm{~m}$. One drift net was set in place at the lower end of each study reach for 24 hours at the beginning and end of each 16-day sampling period. Nets were placed in riffles or runs with the bottom of the net on the streambed and the top above the stream surface such that the entire water column was sampled. Samples were processed in the field and laboratory as described above.

Terrestrial insects and adult aquatic insects that drop into the stream and become part of the drift and potential fish food supply were sampled during each sampling period by means of sticky traps and water traps. A pair (one of each type) was located at each of two sites within each study reach.

Sticky trap-The sticky traps were 0.31 -m squares of white-painted 6.35 -mm plywood; each was covered with a piece of 6-mil clear polyethylene film. This square surface was sprayed with "Tree Tanglefoot," a sticky substance used as a barrier to crawiing insects on trees. Each coated board was taped to a Styrofoam float $0.36 \mathrm{~m}$ square and $5.1 \mathrm{~cm}$ thick. Two sticky traps were set out in each study reach for the full 16 days of each sampling period. When the trap was removed at the end of the sampling period, the plastic film was cut off at the edges of the board so that a $0.31-\mathrm{m}$-square collection surface was retained, butcher paper was placed over the sticky side to prevent crushing or mold damage to the specimens, and this film and butcher paper "sandwich" was transported to the laboratory. In the laboratory, the butcher paper was removed and the film cut into $2.54-\mathrm{cm}$ strips for viewing under a microscope. Insects were counted and identified, usually to family.

\footnotetext{
1) The use of trade, firm, or corporation names in this publication is for the information and convenience of the reader. Such use does not constitute an officiat endorsement or approval by the U.S. Department of Agriculture of any product or service to the exclusion of others that may be suitable.
} 


\section{Sampling Schedule}

Identification of Organisms
Water trap-A water trap was made from a 0.33 - by $0.28-\mathrm{m}$ plastic dishpan, $0.13 \mathrm{~m}$ deep, surrounded by a 0.61 - by $0.61-\mathrm{m}$ rectangle of $5.1-\mathrm{cm}$-thick Styrofoam that supported and floated the pan. The pan was filled to about half its depth with water, and $28.4 \mathrm{~g}$ of formalin and $28.4 \mathrm{~g}$ of a surfactant (Ortho $\mathrm{R} X-77$ Spreader) were added. The surfactant eliminated surface tension and allowed insects to settle to the bottom. $A$ small hole was bored into a lower corner of the pan and was fitted with a rubber stopper for easy removal of the contents. Two water traps were set out in each study reach for the full 16 days. When a trap was removed at the end of a sampling period, the corner plug was removed and the contents of the pan were strained through a 0.5$\mathrm{mm}$ mesh screen. The material remaining on the screen was washed into a jar with formal alcohol and processed as described for benthic and drift samples.

Each stream was sampled during summer and fall 1974 and during all four seasons in 1975 and 1976. Samples were taken at the following times: winter-mid January to early February; spring - early April to late April; summer-early July to late July; and fall-early October to early' November.

A sampling period was organized as follows: On day 1 , sticky traps, water traps, and drift nets were set out. On day 2, 24 hours later, drift nets were pulled. During these 2 days, benthic samples were taken.

Two weeks later, another sampling trip was made. On Day 15, drift nets were set out, and 24 hours later (day 16), they were pulled. Sticky traps and water traps that had been in place during the 16-day period were removed. During these 2 days, benthic samples were again taken.

Because of ice and other weather-related problems, the winter sampling period at the central and eastern sites in both years, and at the Cascade site in 1976, was only one trip of 2 days rather than two trips over 16 days. During these shortened sampling periods, samples from sticky traps and water traps were not obtained, and only half as many of the other samples were collected. We had planned to collect the following samples during the entire study: benthic, 600; drift, 300; and sticky trap and water trap, 280 each. But actual numbers of samples were fewer because on a few occasions sampling gear malfunctioned or was lost.

We used several sources to identify the organisms collected. Invertebrates other than insects were identified through descriptions in Pennak (1978), Ward and Whipple (1959), and Burch (1982). Aquatic insects were identified from taxonomic keys in Hatch (1953, 1957, 1961, 1965, 1971), Usinger (1956), Jensen (1966), Cole (1969), Anderson (1976), Edmunds and others (1976), Baumann and others (1977), and Merritt and Cummins (1978). Terre strial insects were identified primarily from Borror and others (1976). Amphibians and fish were identified from Stebbins (1954) and Bond (1973), respectively. 
Table 1 and figure 2 show the percentage composition by season of the major orders of invertebrates collected in each of the four study areas. In all areas, Diptera was the most abundant order collected in the summer and fall, and mayflies (Ephemeroptera) were prevalent in the winter. In all areas other than central Oregon, spring samples also contained high percentages of mayflies, although springtails (Collembola) dominated the spring samples in central Oregon and stoneflies (Plecoptera) were prevalent in eastern Oregon. In most areas and seasons, Diptera and Ephemeroptera combined comprised over half of the invertebrates collected.

Table 1-Number and percentage composition of invertebrates, by order, for each season and geographic area

\begin{tabular}{|c|c|c|c|c|c|c|c|c|c|c|}
\hline \multirow[t]{2}{*}{$\begin{array}{l}\text { Area and } \\
\text { order }\end{array}$} & \multicolumn{2}{|c|}{ Winter } & \multicolumn{2}{|c|}{ Spring } & \multicolumn{2}{|c|}{ Summer } & \multicolumn{2}{|c|}{ Fall } & \multicolumn{2}{|c|}{ All seasons } \\
\hline & No. & Percent & No. & Percent & No. & Percent & No. & Percent & No. & Percent \\
\hline Coastal: & & & & & & & & & & \\
\hline Gastropoda & 51 & $(1.8)$ & 207 & $(1.7)$ & 1,052 & $(4.6)$ & 615 & $(4.8)$ & 1,925 & (3.8) \\
\hline Collembola & 28 & $(1.0)$ & 477 & (3.9) & 85 & (.4) & 192 & $(1.5)$ & 782 & $(1.5)$ \\
\hline Ephemeroptera & 1,293 & $(46.6)$ & 5,571 & $(45.1)$ & 2.479 & (10.9) & 1,819 & $(14.1)$ & 11,162 & $(21.9)$ \\
\hline Plecoptera & 231 & (8.3) & 1,158 & $(9.4)$ & 1,586 & (6.9) & 1.567 & (12.1) & 4.542 & $(8.9)$ \\
\hline Hemiotera & 6 & $(.2)$ & 85 & $(.7)$ & 233 & $(1.0)$ & $5 !$ & (.4) & 375 & $(.7)$ \\
\hline Homoptera & 5 & $(.2)$ & 32 & (.3) & 241 & (1.1) & 117 & (.9) & 395 & (.8) \\
\hline Coleoptera & 70 & $(2.5)$ & 1.149 & $(9.3)$ & 2.517 & $(11.0)$ & 2.575 & (19.9) & 6,311 & $(12.4)$ \\
\hline Trichoptera & 221 & $(8.0)$ & 676 & (5.5) & 995 & $(4.4)$ & 1.550 & (12.0) & 3.442 & (6.8) \\
\hline Diptera & 832 & $(30.0)$ & 2,777 & $(22.5)$ & 13,149 & $(57.6)$ & 4,108 & (31.8) & 20,866 & $(41.0)$ \\
\hline Other 1 & 35 & (1.3) & 230 & (1.9) & 501 & (2.2) & 330 & $(2.6)$ & 1.096 & $(2.2)$ \\
\hline Total & 2.772 & & 12,362 & & 22,838 & & 12.924 & & 50.896 & \\
\hline \multicolumn{11}{|l|}{ Cascade: } \\
\hline Oligochaeta & 39 & (.8) & 231 & $(1.4)$ & 132 & (.5) & 183 & (1.5) & 585 & $(1.0)$ \\
\hline Collembola & 574 & (12.1) & 564 & (3.4) & 3.031 & (11.1) & 2,333 & $(18.8)$ & 6.502 & $(10.6)$ \\
\hline Ephemeroptera & 1,734 & (36.6) & 9,413 & $(56.0)$ & 4,762 & $(17.4)$ & 2.063 & (16.6) & 17.972 & $(29.3)$ \\
\hline Plecoptera & 1,327 & $(28.0)$ & 2,327 & (13.8) & 1,298 & $(4.8)$ & 2.250 & (18.1) & 7.202 & $(11.8)$ \\
\hline Coleoptera & 28 & (.6) & 89 & (.5) & 832 & (3.0) & 364 & (2.9) & 1,313 & (2.i) \\
\hline Trichoptera & 564 & (11.9) & 607 & (3.6) & 1,311 & (4.8) & 709 & $(5.7)$ & 3,191 & $(5.2)$ \\
\hline Diptera & 414 & $(8.7)$ & 3.478 & $(20.7)$ & 14.563 & $(53.4)$ & 4,022 & $(32.4)$ & 22,477 & $(36.7)$ \\
\hline Other & 52 & (1.1) & 104 & (.6) & 1.367 & $(5.0)$ & 506 & $(4.1)$ & $\underline{2,029}$ & (3.3) \\
\hline Total & 4.732 & & $\overline{16.813}$ & & $\overline{27,296}$ & & $\overline{12,430}$ & & $\overline{61,271}$ & \\
\hline \multicolumn{11}{|l|}{ Central: } \\
\hline Collembola & & & 14,054 & $(49.1)$ & 9.046 & $(24.1)$ & 3.063 & $(10.0)$ & 26,163 & $(25.3)$ \\
\hline Ephemeroptera & 2,076 & (32.3) & 3,444 & (12.0) & 4.816 & (12.8) & 7.464 & (24.3) & 17.800 & $(17.2)$ \\
\hline Plecoptera & 1,865 & $(29.0)$ & 3,585 & (12.5) & 922 & (2.5) & 2.699 & (8.8) & 9.071 & $(6.6)$ \\
\hline Homoptera & & & 69 & (.2) & 1,969 & $(5.2)$ & 1,375 & (4.5) & 3,413 & (3.3) \\
\hline Coleoptera & 205 & $(3.2)$ & 362 & $(1.3)$ & 2,269 & $(6.0)$ & 2.699 & (8.8) & 5.535 & (5.4) \\
\hline Trichoptera & 345 & $(5.4)$ & 1.192 & (4.2) & 2.071 & (5.5) & 2.810 & (9.1) & 6.418 & (6.2) \\
\hline Diptera & 1,873 & $(29.1)$ & 5,657 & (19.8) & 14.839 & (39.5) & 9.777 & (31.8) & 32,146 & $(31.1)$ \\
\hline Hymenoptera & & & 19 & (.1) & 586 & $(1.6)$ & 217 & $(. \overline{1})$ & 822 & (8) \\
\hline Other & 71 & $(1.1)$ & 242 & (.8) & 1.040 & (2.8) & 670 & $(2.2)$ & 2.023 & (2.0) \\
\hline Total & $\overline{6.435}$ & & $\overline{28.624}$ & & $\overline{37.558}$ & & $\overline{30.774}$ & & $\overline{103,391}$ & \\
\hline \multicolumn{11}{|l|}{ Eastern: } \\
\hline Oligochaeta & 34 & (1.9) & 148 & $(1.8)$ & 28 & $(.1)$ & 554 & $(2.2)$ & 764 & (1.3) \\
\hline Gastropoda & 19 & $(1.1)$ & 9 & (.1) & 34 & $(2)$ & 581 & $(2.3)$ & 643 & (1.1) \\
\hline Araneae & & & 8 & (.1) & 272 & $(1.3)$ & 152 & (.6) & 432 & $(.8)$ \\
\hline Collembola & 2 & (.1) & 442 & $(5.4)$ & 12 & (.1) & 245 & $(1.0)$ & 701 & $(1.2)$ \\
\hline Ephemeroptera & 569 & (31.5) & 563 & (6.9) & 3.705 & $(17.2)$ & 5.063 & $(19.7)$ & 9.900 & $(17.3)$ \\
\hline Plecoptera & 446 & $(24.7)$ & 3.984 & $(49.0)$ & 1,099 & $(5.1)$ & 1,342 & $(5.2)$ & 6.871 & (12.0) \\
\hline Hemiptera & 10 & (.6) & 3 & (.0) & 211 & $(1.0)$ & 180 & $(.7)$ & 404 & $(.7)$ \\
\hline Homoptera & 1 & (.1) & 21 & (.3) & 1.002 & $(4.7)$ & 5.426 & $(21.1)$ & 5,450 & (11.3) \\
\hline Coleoptera & 130 & $(7.2)$ & 128 & $(1.6)$ & 1.434 & $(6.7)$ & 1.587 & $(6.2)$ & 3.279 & $(5.7)$ \\
\hline Trichoptera & 124 & (6.9) & 130 & (1.6) & 818 & (3.8) & 1.751 & $(6.8)$ & 2,823 & (4.9) \\
\hline Diptera & 439 & (24.3) & 2.657 & $(32.7)$ & 12.350 & $(57.5)$ & 8.224 & $(32.0)$ & 23.670 & $(41.4)$ \\
\hline Hymenoptera & 1 & (.1) & 4 & (.0) & 229 & (1.1) & 138 & (.5) & 372 & $(.7)$ \\
\hline Other & 30 & (1.7) & 34 & (.4) & 287 & $(1.3)$ & 492 & (1.9) & 843 & (1.5) \\
\hline Total & $\overline{1,805}$ & & 8,131 & & $\overline{21,481}$ & & $\overline{25,735}$ & & $\overline{57,152}$ & \\
\hline
\end{tabular}

1) Other is the total of all orders having a percentage compostion less than $1.0 \mathrm{in}$ any season.

Within 276 families, 426 taxa were collected (appendix 1); the 20 most prevalent families within each sample type accounted for the majority of organisms collected (benthos 89.2 percent, drift 80.4 percent, sticky trap 91.8 percent, water trap 77.8 percent-see table 2). Appendix 2 lists the frequency of occurrence, by family, of organisms collected during the study. 

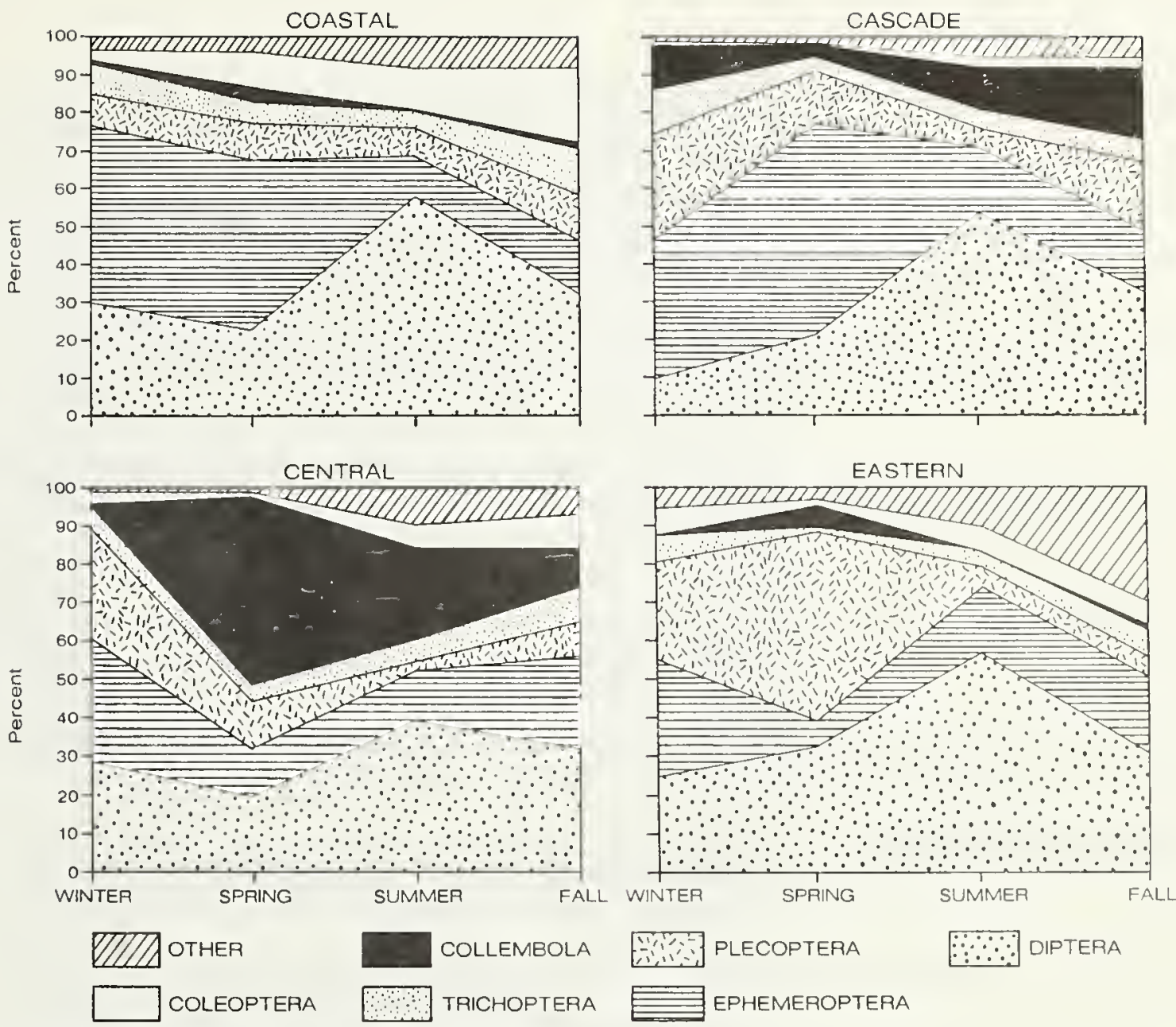

Figure 2-Percentage composition of major orders of invertebrates, by season, for coastal, Cascade, central, and eastern streams in Oregon.

Table 2-Percentage composition of the 20 most prevalent families 1 / collected, by sample type

\begin{tabular}{|c|c|c|c|c|c|c|c|}
\hline \multicolumn{2}{|c|}{ Benthic } & \multicolumn{2}{|l|}{ Drift } & \multicolumn{2}{|c|}{ Slicky trap } & \multicolumn{2}{|c|}{ Water trap } \\
\hline Taxa & Percent & Taxa & Percent & Taxa & Percent & Taxa & Percent \\
\hline Chironomidae & 14.4 & Baetidae & 16.2 & Chironomidae & 19.0 & Chironomidae & 24.4 \\
\hline Heptageniidae & 13.7 & Chironomidae & 15.4 & Dolichopodidae & 12.9 & Isotomidae & 8.0 \\
\hline Elmidae & 10.5 & Nemouridae & 7.2 & Collembola & 9.7 & Empididae & 6.0 \\
\hline Baetidae & 7.0 & Heptageniidae & 5.3 & Empididae & 9.2 & Collembola & 5.5 \\
\hline Chloropertidae & 6.4 & Ephemerellidae & 4.2 & Muscoidea & 7.7 & Ephydridae & 5.1 \\
\hline Ephemerellidae & 6.4 & Limnephilidae & 4.1 & Cicadellidae & 5.4 & Mycetophılidae & 4.2 \\
\hline Tipulidae & 4.0 & Pleuroceridae & 3.7 & Trichoptera & 4.1 & Sciaridae & 2.9 \\
\hline Leptophlebiidae & 3.8 & Simuliidae & 3.0 & Isotomidae & 3.6 & Tipulidae & 2.6 \\
\hline Nemouridae & 3.3 & Capniidae & 2.8 & Mycetophilidae & 3.5 & Dolichopodidae & 2.5 \\
\hline Perlıdae & 2.8 & Siphlonuridae & 2.3 & Nemouridae & 3.1 & Nemouridae & 2.5 \\
\hline Oligochaeta & 2.5 & Sicadellidae & 2.3 & Diptera & 2.6 & Araneae & 2.3 \\
\hline Psychodidae & 2.4 & Elmidae & 2.3 & Tipulidae & 2.0 & Aphididae & 2.3 \\
\hline Limnephilidae & 2.2 & Leptophlebiidae & 1.9 & Sciaridae & 1.9 & Staphylinıdae & 1.6 \\
\hline Siphlonuridae & 2.0 & Sciaridae & 1.7 & Ephemeroptera & 1.9 & Ceratopogonıdae & 1.4 \\
\hline Rhyacophilidae & 1.4 & Amnicolidae & 1.6 & Platypezıdae & 1.3 & Cecidomyiidae & 1.3 \\
\hline Perlodidae & 1.4 & Calamoceratidae & 1.2 & Staphylinidae & 1.3 & Cicadellidae & 1.3 \\
\hline Hydropsychidae & 1.3 & Chloroperlidae & 1.2 & Plecoptera & .9 & Capniidae & 1.1 \\
\hline Capniidae & 1.3 & Ephemeroptera & 1.0 & Araneae & .7 & Phoridae & 1.1 \\
\hline Pteronarcidae & 1.2 & Psychodidae & 1.0 & Ephydridae & .6 & Anthomyiidae & .9 \\
\hline Pleuroceridae & 1.2 & Aphididae & 1.0 & Chloroperlidae & 4 & Simuliidai & .8 \\
\hline Total & $\overline{89.2}$ & & 79.4 & & 91.8 & & 77.8 \\
\hline
\end{tabular}

II A few higher taxa are included where identification could not be made to the family level. 


\section{English Equivalents}

\section{Literature Cited}

When the data are divided by sample type, seasonal and geographic trends become apparent (appendix 3). Within the benthic community, Diptera were more prevalent in summer and fall than in winter or spring in Cascade streams, while in coastal streams they were more prevalent in fall; in central and eastern streams, Diptera were dominant in spring samples. Within the drift, Diptera showed no obvious trend across season or geographic area. Diptera was also the most prevalent order collected in sticky-trap and water-trap samples except for occasional large numbers of Collembola. Ephemeroptera within the benthos were most common during winter and spring sampling; within the drift, their presence peaked during spring and summer sample periods in all areas except eastern Oregon, where they were most numerous in the winter samples. Adult mayflies were never abundant in sticky-trap or water-trap samples. Plecoptera were an important component of winter and spring drift samples collected in eastern Oregon; adult stoneflies also dominated spring water-trap samples in this area. Collembola were occasionally important in all areas except coastal Oregon, but particularly in central Oregon where they comprised over 25 percent of all organisms collected. Collembola were found primarily in sticky-trap and water-trap samples and generally during spring and fall.

1 meter $(m)=3.28$ feet

1 square meter $\left(m^{2}\right)=10.76$ square feet

1 centimeter $(\mathrm{cm})=0.39$ inch

1 millimeter $(\mathrm{mm})=0.039$ inch

1 micrometer $(\mu)=0.000039$ inch

1 gram $(g)=0.035$ ounce

Anderson, Norman $\mathrm{H}$. The distribution and biology of the Oregon Trichoptera. Tech. Bull. 134. Corvallis, OR: Oregon State University, Agricultural Experiment Station; 1976. $152 \mathrm{p}$.

Baumann, Richard W.; Gaufin, Arden R.; Surdick, Rebecca F. The stoneflies (Plecoptera) of the Rocky Mountains. Memoirs of the American Entomological Society. 31: 1-208; 1977.

Bond, Carl E. Keys to Oregon freshwater fishes. Tech. Bull. 58. Corvallis, OR: Oregon State University, Agricultural Experiment Station; 1973. 42 p.

Borror, Donald J.; Delong, Dwight M.; Triplehorn, Charles A. An introduction to the study of insects. 4th ed. New York, NY: Holt, Rinehart and Winston; 1976. 852 p.

Burch, J.B. Freshwater snails (Mollusca: Gastropoda) of North America. EPA-600/382-026. Cincinnati, OH: U.S. Environmental Protection Agency; 1982. 294 p.

Cole, Frank R. The flies of western North America. Berkeley, CA: University of California Press; 1969. 693 p.

Edmunds, George F., Jr.; Jensen, Steven L.; Berner, Lewis. The mayflies of North and Central America. Minneapolis, MN: University of Minnesota Press; 1976.330 p.

Hatch, Melville $\mathrm{H}$. The beetles of the Pacific Northwest. Part I: Introduction and Adephaga. Seattle, WA: University of Washington Press; 1953. 340 p. 
Hatch, Melville H. The beetles of the Pacific Northwest. Part II: Staphyliniformia. Seattle, WA: University of Washington Press; 1957. 384 p.

Hatch, Melville H. The beetles of the Pacific Northwest. Part III: Pselaphidae and Diversicornia I. Seattle, WA: University of Washington Press; 1961.503 p.

Hatch, Melville H. The beetles of the Pacific Northwest. Part IV: Macrodactyles, Palpicornes and Heteromera. Seattle, WA: University of Washington Press; 1965. $268 \mathrm{p}$.

Hatch, Melville $H$. The beetles of the Pacific Northwest. Part V: Rhipiceroidea, Sternoxi, Phytophaga, Rhynchophora and Lamellicornia. Seattle, WA: University of Washington Press; 1971. 662 p.

Jensen, Stephen Leroy. The mayflies of Idaho (Ephemeroptera). Salt Lake City, UT: University of Utah; 1966. 352 p. M.S. thesis.

Merritt, Richard W.; Cummins, Kenneth W., eds. An introduction to the aquatic insects of North America. Dubuque, IA: Kendall/Hunt Publishing Co.; 1978. 411 p.

Pennak, Robert W. Fresh-water invertebrates of the United States. $2 d$ ed. New York, NY: John Wiley and Sons; 1978. 803 p.

Stebbins, Robert C. Amphibians and reptiles of western North America. New York, NY: McGraw-Hill Book Co.; 1954. 528 p.

Usinger, Robert L., ed. Aquatic insects of California. Berkeley, CA: University of California Press; 1956.508 p.

Ward, Henry Baldwin; Whipple, George Chandler. Fresh-water biology. 2 d ed. New York, NY: John Wiley and Sons; 1959. 1248 p. 


\section{Appendix 1}

Presence of Taxa by Season and Area
Individuals were identified to the lowest taxonomic level. Presence was recorded at this level and not entered at the broader taxonomic levels.

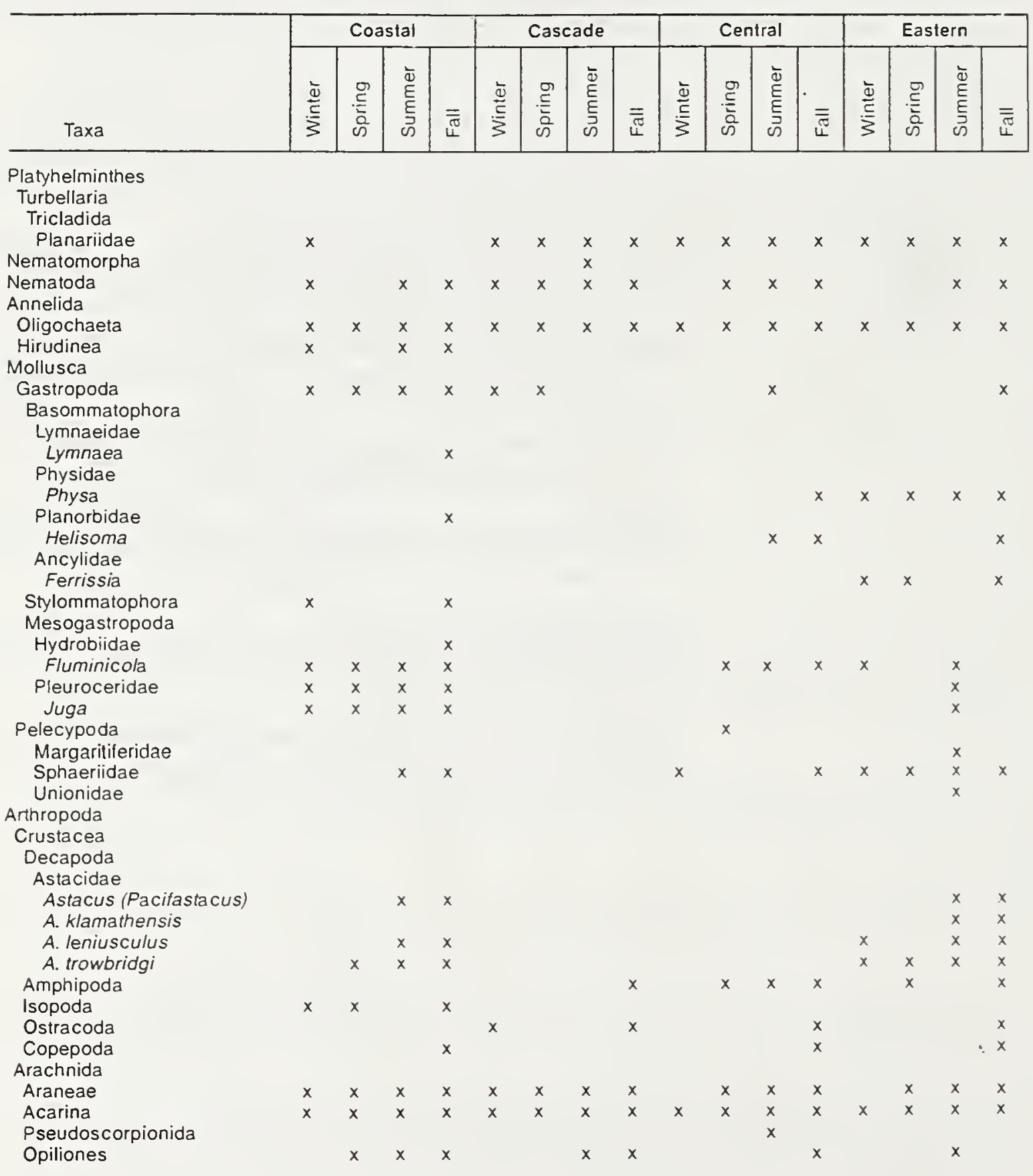

Table continued on next page. 


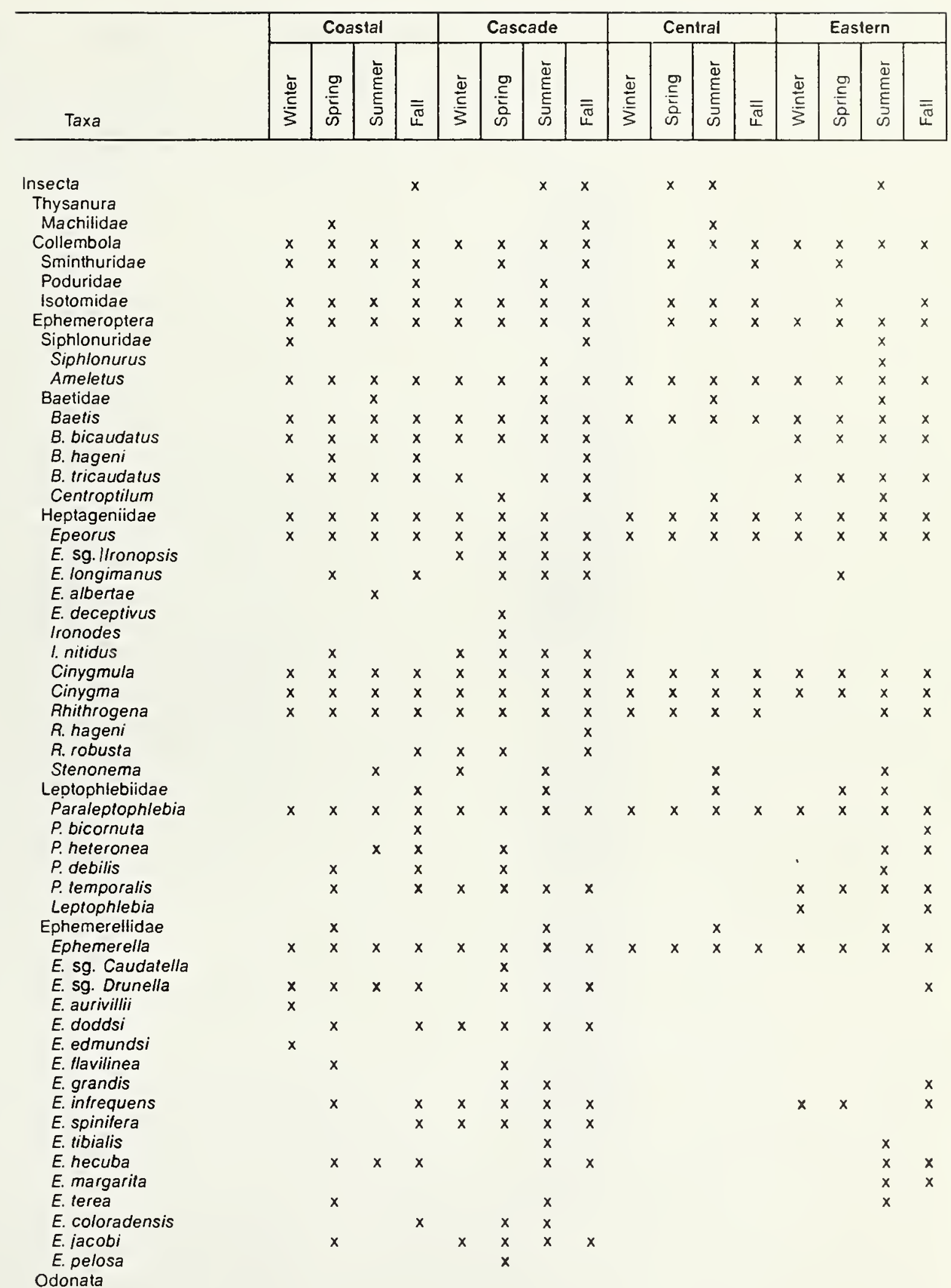

Table continued on next page. 


\begin{tabular}{|c|c|c|c|c|c|c|c|c|c|c|c|c|c|c|c|c|}
\hline \multirow[b]{2}{*}{ Taxa } & \multicolumn{4}{|c|}{ Coastal } & \multicolumn{4}{|c|}{ Cascade } & \multicolumn{4}{|c|}{ Central } & \multicolumn{4}{|c|}{ Eastern } \\
\hline & $\stackrel{\stackrel{D}{\leftrightarrows}}{3}$ & 号 & $\stackrel{\bar{d}}{E}$ & $\overline{\bar{\varpi}}$ & $\stackrel{\stackrel{D}{E}}{\stackrel{5}{\zeta}}$ & 号 & $\stackrel{\stackrel{D}{E}}{\sum_{J}^{E}}$ & $\overline{\bar{\sigma}}$ & $\frac{\grave{D}}{\stackrel{\Xi}{\Xi}}$ & 은 & $\stackrel{\text { E् }}{\stackrel{\Xi}{\Xi}}$ & $\overline{\bar{\varpi}}$ & $\frac{\stackrel{2}{\Phi}}{\stackrel{5}{S}}$ & 号 & 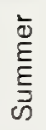 & $\overline{5}$ \\
\hline Anisoptera & & & $x$ & & & & $x$ & & & & & & & & $x$ & \\
\hline $\begin{array}{l}\text { Aeshnidae } \\
\text { Anax }\end{array}$ & & & & & - & & & & & & & & & & & $\begin{array}{l}x \\
x\end{array}$ \\
\hline $\begin{array}{l}\text { Gomphidae } \\
\text { Ophiogomphus }\end{array}$ & & & $x$ & & & & & & & & $x$ & & & & & $x$ \\
\hline Cordulegastridae & & & & & & & & & $x$ & & & $x$ & & & & \\
\hline $\begin{array}{l}\text { Libellulidae } \\
\text { Coenagrıonidae }\end{array}$ & & & & & & & & & & $x$ & & & & & & \\
\hline Argia & & & $x$ & & & & & & & & & & & & $x$ & \\
\hline Orthoptera & & $x$ & & & & & & & & & $x$ & & & & $x$ & $x$ \\
\hline Tetrigidae & & & & & & & & & & $x$ & & $x$ & & & & \\
\hline Gryllidae & & & & & & & & & & & $x$ & & & & $x$ & \\
\hline Phaneropterinae & & & & $x$ & & & & & & & & & & & & \\
\hline Gryllacrididae & & $x$ & $x$ & $x$ & & $x$ & $x$ & $x$ & & & & & & & & \\
\hline Acrididae & & & & & & & $x$ & $x$ & & & & & & & $x$ & \\
\hline Dermaptera & & & $x$ & & & & & & & & & & & & & \\
\hline Forficulidae & & & & & & & & & & & & $x$ & & & & \\
\hline Plecoptera & $x$ & $x$ & $x$ & $x$ & $x$ & $x$ & $x$ & $x$ & & $x$ & $x$ & $x$ & $x$ & $x$ & $x$ & $x$ \\
\hline Pteronarcidae & & $x$ & $x$ & & & & $x$ & & & & & & & & & \\
\hline Pteronarcella & & & $x$ & $x$ & & & & $x$ & & & & & & & & \\
\hline Pteronarcys & & & $x$ & $x$ & & $x$ & $x$ & $x$ & & & & & $x$ & & & \\
\hline Peltoperlidae & & & & & & & & & & & & & & & & \\
\hline Yoraperla & & & $x$ & $x$ & $x$ & $x$ & $x$ & $x$ & $x$ & $x$ & $x$ & $x$ & & & & \\
\hline Taeniopterygidae & & & & & & & & & & & & & & & & \\
\hline Brachypterıgınae & $x$ & $x$ & $x$ & $x$ & $x$ & $x$ & $x$ & $x$ & & & $x$ & $x$ & & $x$ & & $x$ \\
\hline Nemouridae & $x$ & $\hat{x}$ & $x$ & $x$ & $x$ & $x$ & $x$ & $x$ & $x$ & $x$ & $x$ & $x$ & $x$ & $x$ & $x$ & $x$ \\
\hline Zapada & $\hat{x}$ & $\hat{x}$ & $x$ & $x$ & $x$ & $x$ & $x$ & $x$ & & & & & $x$ & & $x$ & $x$ \\
\hline Malenka & & $x$ & & $x$ & $x$ & $\hat{x}$ & $\hat{x}$ & $\hat{x}$ & & & & & $x$ & $x$ & $x$ & $x$ \\
\hline Capniidae & $x$ & $x$ & $x$ & $x$ & $x$ & $x$ & $x$ & $x$ & $x$ & $x$ & $x$ & $x$ & $x$ & $x$ & $x$ & $x$ \\
\hline Capnia & & & & $x$ & $x$ & $x$ & $x$ & $x$ & & $x$ & & & $x$ & $x$ & $x$ & $x$ \\
\hline Isocapnia & & & & & & $x$ & & & & & & & & & & \\
\hline Eucapnopsis & $x$ & & $x$ & & $x$ & $x$ & & & $x$ & & & $x$ & & $x$ & & \\
\hline $\begin{array}{l}\text { Leuctridae } \\
\text { Despaxia }\end{array}$ & $x$ & $x$ & $x$ & $x$ & $x$ & $x$ & $x$ & $x$ & $x$ & $x$ & & $x$ & & $x$ & & \\
\hline D. augustus & & & & & $x$ & $x$ & & $x$ & & & & & & & & \\
\hline $\begin{array}{l}\text { Perlidae } \\
\text { Calineuria }\end{array}$ & $x$ & $x$ & $x$ & $x$ & $x$ & $x$ & $x$ & $x$ & $x$ & $x$ & $x$ & $x$ & $x$ & $x$ & $x$ & $x$ \\
\hline C. californica & $x$ & $x$ & $x$ & $x$ & $x$ & $x$ & $x$ & $x$ & & & & & $x$ & $x$ & $x$ & $x$ \\
\hline Claassenia & & $x$ & $x$ & & & & & & $x$ & $x$ & $x$ & $x$ & & & & \\
\hline Hesperoperla & $x$ & $x$ & $x$ & $x$ & & & & $x$ & & & & & & & & \\
\hline Perlodidae & $x$ & $x$ & $x$ & $x$ & & $x$ & $x$ & $x$ & & & $x$ & & $x$ & & $x$ & $x$ \\
\hline Perlodinae & $x$ & $x$ & $x$ & $x$ & $x$ & $x$ & $x$ & $x$ & $x$ & $x$ & $x$ & $x$ & $x$ & $x$ & $x$ & $x$ \\
\hline Isoperla & $\hat{x}$ & $x$ & $x$ & $\hat{x}$ & & $x$ & & $x$ & $x$ & $x$ & $x$ & $x$ & & $x$ & $x$ & \\
\hline Perlinodes & & & & & & & & & & & & & $x$ & & & $x$ \\
\hline Skwala & & & & $x$ & $x$ & $x$ & $x$ & $x$ & & & & & & & & $\hat{x}$ \\
\hline Chloroperlidae & $x$ & $x$ & $x$ & $x$ & $x$ & $x$ & $x$ & $x$ & $x$ & $x$ & $x$ & $x$ & $x$ & $x$ & $x$ & \\
\hline Chloroperlinae & $x$ & $x$ & $x$ & $x$ & $x$ & $x$ & $x$ & $x$ & $x$ & $x$ & $x$ & $x$ & $x$ & $x$ & $x$ & $x$ \\
\hline Hastaperla & & & $x$ & & & & & & & & & & & & & \\
\hline
\end{tabular}

Table continued on next page. 


\begin{tabular}{|c|c|c|c|c|c|c|c|c|c|c|c|c|c|c|c|c|}
\hline \multirow[b]{2}{*}{ Taxa } & \multicolumn{4}{|c|}{ Coastal } & \multicolumn{4}{|c|}{ Cascade } & \multicolumn{4}{|c|}{ Central } & \multicolumn{4}{|c|}{ Eastern } \\
\hline & $\frac{\frac{d}{c}}{3}$ & 号 & 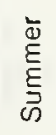 & $\stackrel{\overline{\widetilde{W}}}{\stackrel{\bar{W}}{ }}$ & $\frac{\bar{d}}{\stackrel{5}{5}}$ & $\begin{array}{l}\stackrel{0}{5} \\
\text { की }\end{array}$ & 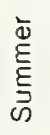 & $\overline{\bar{\pi}}$ & $\frac{\grave{\Phi}}{\sum}$ & $\begin{array}{l}\text { 든 } \\
\text { 응 }\end{array}$ & 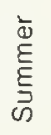 & $\overline{\bar{\varpi}}$ & $\stackrel{\frac{2}{9}}{\stackrel{5}{\zeta}}$ & $\frac{0}{5}$ & $\frac{\stackrel{\Xi}{E}}{\stackrel{E}{\Xi}}$ & $\overline{\overline{\mathscr{C}}}$ \\
\hline \multicolumn{17}{|l|}{ Paraperlinae } \\
\hline Paraperla & & $x$ & $x$ & $x$ & & & $x$ & & & & & & & & & \\
\hline Kathroperla & & & & & $x$ & & $x$ & $x$ & & & & & & & & \\
\hline Psocoptera & & & $x$ & $x$ & & & $x$ & $x$ & & & $x$ & $x$ & & & $x$ & $x$ \\
\hline Mallophaga & & & $x$ & & & & $x$ & $x$ & & & & & & & & \\
\hline Thysanoptera & & $x$ & $x$ & $x$ & & & $x$ & & & $x$ & $x$ & $x$ & & & $x$ & $x$ \\
\hline Hemiptera & & $x$ & $x$ & $x$ & $x$ & & $x$ & $x$ & & $x$ & $x$ & $x$ & $x$ & & $x$ & \\
\hline Corixidae & & & & & & & & & $x$ & & $x$ & $x$ & $x$ & & $x$ & $x$ \\
\hline Graptocorixa & & & & & & & & & & & & & & & & $x$ \\
\hline Hesperocorixa & & & & & & & & & & & & & $x$ & & & $x$ \\
\hline Trichocorixa & & & & & & & & & & & & & & & & $x$ \\
\hline Neididae & & & & & & & & & & & $x$ & & & & & \\
\hline Macroveliidae & & & & & & & & & & & & & & & & \\
\hline Macrovelia & & & & $x$ & & & & & & & & & & & & \\
\hline Gerridae & & $x$ & $x$ & $x$ & & & $x$ & $x$ & & $x$ & $x$ & $x$ & & & $x$ & $x$ \\
\hline Gerris & $x$ & $x$ & $x$ & $x$ & & $x$ & $x$ & $x$ & & $x$ & $x$ & & & $x$ & $x$ & $x$ \\
\hline Trepobates & & & $x$ & & & & & & & & $x$ & & & & $x$ & $x$ \\
\hline Veliidae & & $x$ & $x$ & $x$ & & & & & & & $x$ & $x$ & & & & \\
\hline Microvelia & & $x$ & $x$ & $x$ & & & & & & & & & & & & \\
\hline Mesoveliidae & & $x$ & & & & & & & & & & & & & & \\
\hline Saldidae & & $x$ & $x$ & & & & $x$ & $x$ & & & $x$ & $x$ & & & $x$ & $x$ \\
\hline Anthocoridae & & & & & & & & & & & $x$ & & & & $x$ & \\
\hline Miridae & & $x$ & $x$ & $x$ & & & $x$ & $x$ & & & $x$ & $x$ & & & $x$ & \\
\hline Nabidae & & & & & & & $x$ & & & & $x$ & & & & $x$ & $x$ \\
\hline Reduviidae & & & & & & & & & & & $x$ & $x$ & & & & $x$ \\
\hline Tingidae & & $x$ & $x$ & $x$ & & & $x$ & & & & $x$ & & & & & \\
\hline Aradidae & & & $x$ & & & & & & & & $x$ & & & & & \\
\hline Coreidae & & & & & & & $x$ & & & & & & & & & $x$ \\
\hline Lygaeidae & & $x$ & $x$ & $x$ & & & $x$ & & & & $x$ & & & & $x$ & \\
\hline Pentatomidae & & & $x$ & $x$ & & & & & & & & & & & & \\
\hline Homoptera & $x$ & $x$ & $x$ & $x$ & & & $x$ & $x$ & & $x$ & $x$ & $x$ & & $x$ & $x$ & $x$ \\
\hline Cicadellidae & $x$ & $x$ & $x$ & $x$ & & $x$ & $x$ & $x$ & & $x$ & $x$ & $x$ & $x$ & $x$ & $x$ & $x$ \\
\hline Cercopidae & & & $x$ & $x$ & & & $x$ & $x$ & & $x$ & $x$ & $x$ & & $x$ & $x$ & $x$ \\
\hline Delpracidae & & & & & & $x$ & $x$ & & & & $x$ & & & & $x$ & \\
\hline Achilidae & & & & & & & $x$ & & & & & & & & & \\
\hline Psyllidae & & $x$ & $x$ & $x$ & & $x$ & $x$ & $x$ & & $x$ & $x$ & $x$ & & $x$ & & $x$ \\
\hline Aphididae & $x$ & $x$ & $x$ & $x$ & & & $x$ & $x$ & & $x$ & $x$ & $x$ & & $x$ & $x$ & $x$ \\
\hline Eriosomatidae & & & $\hat{x}$ & & & & & & & & $x$ & $x$ & & & & $x$ \\
\hline Chermidae & & & & $x$ & & & & & & & & & & & & \\
\hline Coccoidea & & $x$ & & $x$ & & & & & & $x$ & & $x$ & & & & \\
\hline Coleoptera & $x$ & $x$ & $x$ & $x$ & $x$ & & $x$ & $x$ & & $x$ & $x$ & $x$ & $x$ & $x$ & $x$ & $x$ \\
\hline Cupedidae & & & & & & & & & & & $x$ & & & & & \\
\hline Silphidae & & & & & & & & & & & $x$ & & & & & \\
\hline Lathridiidae & & $x$ & & $x$ & & & $x$ & & & & $x$ & & & & & \\
\hline Noteridae & & & & & & & $x$ & $x$ & & & & & & & & \\
\hline Pronotenus & & & & & & & & & & & & & & & $x$ & \\
\hline Carabidae & & $x$ & $x$ & $x$ & & & $x$ & & & $x$ & $x$ & $x$ & & & $x$ & $x$ \\
\hline Haliplidae & & & & & & & & & & & $x$ & $x$ & & & $x$ & $x$ \\
\hline Brychius & & & & & & & & & & & & & $x$ & & $x$ & $x$ \\
\hline Amphizoidae & & & $x$ & $x$ & $x$ & & $x$ & $x$ & & & $x$ & $x$ & & & & \\
\hline Dytiscidae & $x$ & $x$ & $x$ & $x$ & & $x$ & $x$ & $x$ & $x$ & $x$ & $x$ & $x$ & $x$ & $x$ & $x$ & $x$ \\
\hline Deronectes & & $x$ & $x$ & & & & & & & & & & & $x$ & & $x$ \\
\hline Oreodytes & & $x$ & $x$ & & & & $x$ & & & & $x$ & & & & $x$ & \\
\hline
\end{tabular}

Table continued on next page. 


\begin{tabular}{|c|c|c|c|c|c|c|c|c|c|c|c|c|c|c|c|c|}
\hline \multirow[b]{2}{*}{ Taxa } & \multicolumn{4}{|c|}{ Coastal } & \multicolumn{4}{|c|}{ Cascade } & \multicolumn{4}{|c|}{ Central } & \multicolumn{4}{|c|}{ Eastern } \\
\hline & 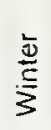 & 号 & $\begin{array}{l}\stackrel{ \pm}{E} \\
\text { E }\end{array}$ & $\overline{\bar{\pi}}$ & $\stackrel{\frac{\vdots}{\infty}}{3}$ & 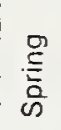 & $\stackrel{\stackrel{\text { ¿ }}{E}}{\stackrel{\Xi}{J}}$ & $\overline{\bar{\varpi}}$ & $\frac{\frac{\grave{\Phi}}{5}}{3}$ & $\frac{\square}{5}$ & $\stackrel{\stackrel{\llcorner}{\Phi}}{\stackrel{L}{E}}$ & $\overline{\bar{\pi}}$ & $\frac{\stackrel{\Phi}{5}}{3}$ & $\begin{array}{l}\stackrel{g}{5} \\
\text { 号 }\end{array}$ & 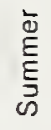 & $\overline{\bar{\mho}}$ \\
\hline Agabus & & $x$ & & & & & & & & & & & & & $x$ & \\
\hline Bidessus & & & & & & & & & & & & & & & $x$ & $x$ \\
\hline Hydroporinae & & & & & & & $x$ & & & & & & & & & \\
\hline Hydroporus & & & & & & & & $x$ & & & & & & & $x$ & \\
\hline Hydrovatus & & $x$ & $x$ & $x$ & & & $x$ & $x$ & & & & & $x$ & & $x$ & $x$ \\
\hline Melyridae & & & $x$ & & & $x$ & & & & & & & & & & \\
\hline Gyrinidae & $x$ & & & & & & & & & & & $x$ & & & & \\
\hline Histeridae & & & $x$ & & & & $x$ & & & & & & & & $x$ & $x$ \\
\hline Hydrophilidae & & $x$ & $x$ & $x$ & $x$ & $x$ & $x$ & $x$ & $x$ & $x$ & $x$ & $x$ & $x$ & $x$ & $x$ & $x$ \\
\hline Paracymus & & & $x$ & & & & $x$ & & & & & & & & & \\
\hline Crenitis & & $x$ & $x$ & & & & $x$ & & & & $x$ & & & & $x$ & \\
\hline Laccobius & & & & $x$ & & & $x$ & & & $x$ & $x$ & $x$ & & $x$ & $x$ & \\
\hline Ametor & & & & $x$ & & $x$ & $x$ & & & & $x$ & & & & & \\
\hline Berosus & & & & & & & & & & & $x$ & & & & $x$ & \\
\hline Tropisternus & & & & & & & & & & & $x$ & & & & & \\
\hline Helophorus & & & & $x$ & $x$ & $x$ & & & & & & & & $x$ & $x$ & $x$ \\
\hline Hydrobius & & & & & & & $x$ & & & & & & & & & \\
\hline Hydraenidae (Limnebiidae) & & $x$ & & & $x$ & $x$ & $x$ & $x$ & $x$ & $\mathrm{x}$ & $x$ & $x$ & $x$ & $x$ & $x$ & $x$ \\
\hline Hydraena & & $x$ & & & & & & $x$ & & $x$ & $x$ & $x$ & $x$ & $x$ & $x$ & $x$ \\
\hline Ochthebius & & & $x$ & & $x$ & & & & & & & $x$ & $x$ & $x$ & $x$ & $x$ \\
\hline Melandryidae & & & & & & & $x$ & & & & $x$ & & & & $x$ & \\
\hline Scarabaeidae & & & & & & & $x$ & & & & $x$ & & & & $x$ & \\
\hline Cicindelidae & & & & & & & $x$ & & & & & & & & & \\
\hline Ptiliidae & & $x$ & $x$ & $x$ & & & $x$ & & & & $x$ & $x$ & & & $x$ & $x$ \\
\hline Chrysomelidae & $x$ & $x$ & $x$ & $x$ & & & $x$ & $x$ & & $x$ & $x$ & $x$ & & & $x$ & $x$ \\
\hline Scaphidiidae & & & & & & & & & & & $x$ & $x$ & & & & \\
\hline Staphylinidae & $x$ & $x$ & $x$ & $x$ & $x$ & $x$ & $x$ & $x$ & & $x$ & $x$ & $x$ & $x$ & $x$ & $x$ & $x$ \\
\hline Pselaphidae & & & & & & & $x$ & & & & & & & & & \\
\hline Colydiidae & & & $x$ & $x$ & & & & & & & $x$ & & & & & \\
\hline Scydmaenidae & & & & & & & & & & & & $x$ & & & $x$ & \\
\hline Meloidae & & & & & & & $x$ & & & & & & & & & \\
\hline Cantharidae & & $x$ & $x$ & & & & $x$ & & & & $x$ & & & & $x$ & \\
\hline Lampyridae & & & & & & & $x$ & & & & & & & & $x$ & \\
\hline Curculionidae (Nemo- & & & & $x$ & & & $x$ & $x$ & & $x$ & $x$ & $x$ & & & & $x$ \\
\hline $\begin{array}{l}\text { nychioae) } \\
\text { Malachiidae }\end{array}$ & & $x$ & $x$ & $x$ & & & $x$ & $x$ & & $x$ & $\hat{x}$ & $\lambda$ & & & & \\
\hline Cleridae & & & & & & & $x$ & & & $x$ & $x$ & $x$ & & & & \\
\hline Elateridae & & & $x$ & $x$ & $x$ & & $x$ & & & & $x$ & & & & $x$ & \\
\hline Eucnemidae & & & & & & & $x$ & & & & & & & & & \\
\hline Byrrhidae & $x$ & & & $x$ & & & & & & & & & & & & \\
\hline Buprestidae & & & & & & & $x$ & & & & $x$ & $x$ & & & & \\
\hline Dascillidae & & & $x$ & & & & $x$ & & & & & & & & & \\
\hline Ptilodactylidae & & & & & & $x$ & & & & & & & & & & \\
\hline Helodidae & & & & & & $x$ & & & & & & $x$ & & & & \\
\hline Dryopidae & & & & & & & & & & & $x$ & $x$ & & & & \\
\hline Limnichidae & & & & & & & $x$ & & & & & & & & & \\
\hline $\begin{array}{l}\text { Psephenidae } \\
\text { Acneus }\end{array}$ & & & & & & & $x$ & & & & & & & & & $x$ \\
\hline A. quadrimaculatus & & & & & & & $x$ & & & & & & & & & \\
\hline Elmidae & $x$ & $x$ & $x$ & $x$ & $x$ & $x$ & $x$ & $x$ & $x$ & $x$ & $x$ & $x$ & $x$ & $x$ & $x$ & $x$ \\
\hline Heterlimnius & $x$ & $x$ & $x$ & $x$ & & $x$ & $x$ & $x$ & & & $x$ & $x$ & & & $x$ & \\
\hline Optioservus & $\hat{x}$ & $\hat{x}$ & $\hat{x}$ & $\hat{x}$ & & $\hat{x}$ & $\hat{x}$ & $x$ & & & & & $x$ & $x$ & $x$ & $x$ \\
\hline
\end{tabular}

Table continued on next page. 


\begin{tabular}{|c|c|c|c|c|c|c|c|c|c|c|c|c|c|c|c|c|}
\hline \multirow[b]{2}{*}{ Taxa } & \multicolumn{4}{|c|}{ Coastal } & \multicolumn{4}{|c|}{ Cascade } & \multicolumn{4}{|c|}{ Central } & \multicolumn{4}{|c|}{ Eastern } \\
\hline & $\frac{\stackrel{\bar{\omega}}{\leftrightarrows}}{\xi}$ & 号 & 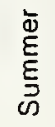 & $\overline{\bar{\pi}}$ & 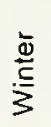 & $\begin{array}{l}\text { 든 } \\
\text { ڤั }\end{array}$ & $\begin{array}{l}\text { à } \\
\stackrel{5}{E} \\
\stackrel{5}{S} \\
0\end{array}$ & $\overline{\bar{\sigma}}$ & $\frac{\stackrel{\grave{\omega}}{\leftrightarrows}}{3}$ & 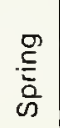 & $\stackrel{\stackrel{\llcorner}{E}}{\stackrel{E}{E}}$ & $\overline{\bar{\pi}}$ & $\frac{\stackrel{\bigsqcup}{\Phi}}{\vdots}$ & 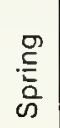 & $\begin{array}{l}\stackrel{\bar{\Phi}}{E} \\
\underline{\Xi} \\
\bar{D}\end{array}$ & $\overline{\bar{\pi}}$ \\
\hline Heterlimnius-optioservus & & $x$ & $x$ & $x$ & & & & $x$ & & & $x$ & & & & $x$ & $x$ \\
\hline $\begin{array}{l}\text { Narpus } \\
\text { N. concolor }\end{array}$ & & & $x$ & $x$ & & $x$ & $x$ & $x$ & & & $x$ & & & & & \\
\hline Zaitzevia & & $x$ & $x$ & & & & & & & & $x$ & & $x$ & & $x$ & $x$ \\
\hline Lara & $x$ & $x$ & $x$ & $x$ & $x$ & $x$ & $x$ & $x$ & & & $x$ & & & & & $x$ \\
\hline Microcylloepus & & & & & & & & & & & $x$ & $x$ & & & & \\
\hline Cleptelmis & & & & & & & & $x$ & & & $x$ & $x$ & $x$ & & & $x$ \\
\hline Dubiraphia & & & & & & & & & & & & $x$ & & & & \\
\hline $\begin{array}{l}\text { Ordobrevia } \\
\text { Ampumixis }\end{array}$ & & & & & & & & & & & & & & & $x$ & $\begin{array}{l}x \\
x\end{array}$ \\
\hline Scolytidae & & $x$ & $x$ & & & & $x$ & & & & $x$ & $x$ & & $x$ & $x$ & $x$ \\
\hline Derodontidae & & & & & & & $x$ & $x$ & & & $x$ & $x$ & & & & \\
\hline Cryptophagidae & & $x$ & & & & & $x$ & & & & & & & & & \\
\hline Bostrichidae & & & & & & & $x$ & & & & & & & & $x$ & \\
\hline Nitidulidae & & $x$ & $x$ & & & & $x$ & $x$ & & $x$ & $x$ & $x$ & & & $x$ & \\
\hline Cucujidae & & & & & & & $x$ & & & & & & & & & \\
\hline Endomychidae & $x$ & & & & & & $x$ & & & & & & & & & \\
\hline Coccinellidae & & & $x$ & $x$ & & & $x$ & & & & $x$ & & & & $x$ & $x$ \\
\hline Cerambycidae & & $x$ & & & & & $x$ & & & & $x$ & & & & $x$ & \\
\hline Anthicidae & & & $x$ & & & & $x$ & & & & & & & & & \\
\hline Pedilidae & & & & & & & & & & & & $x$ & & & & \\
\hline Cephaloidae & & & & & & & & & & & $x$ & & & & & \\
\hline Mordellidae & & & & & & & $x$ & & & & $x$ & & & & . & \\
\hline Tenebrionidae & & & & & & & $x$ & & & & $x$ & & & & & \\
\hline Neuroptera & & $x$ & & & & $x$ & & $x$ & & & & $x$ & & & $x$ & \\
\hline $\begin{array}{l}\text { Megaloptera } \\
\text { Sialidae }\end{array}$ & & & & & & & & & & & & & & & & \\
\hline Sialis & & & $x$ & $x$ & $x$ & & $x$ & $x$ & & $x$ & & & $x$ & $x$ & $x$ & $x$ \\
\hline Corydalidae & & & & & $x$ & $x$ & $x$ & $x$ & & & & & & & & \\
\hline Dysmicohermes & & & $x$ & & $x$ & $x$ & $x$ & & & & & & & & & \\
\hline Raphıdioptera & & & & & & & & & & & & & & & & \\
\hline Raphidiidae & & & & & & & & & & & $x$ & & & & & \\
\hline Planipennia & & & & & & & & & & & & & & & & \\
\hline Coniopterygidae & & & & & & & & & & & $x$ & & & & & \\
\hline Hemerobiidae & & & $x$ & & & $x$ & & & & & & $x$ & & & & \\
\hline Chrysopidae & & & & & & $x$ & $x$ & & & & & & & & $x$ & \\
\hline Trichoptera & $x$ & $x$ & $x$ & $x$ & $x$ & $x$ & $x$ & $x$ & $x$ & $x$ & $x$ & $x$ & & $x$ & $x$ & $x$ \\
\hline Limnephilidae & $x$ & $x$ & $x$ & $x$ & $x$ & $x$ & $x$ & $x$ & $x$ & $x$ & $x$ & $x$ & $x$ & $x$ & $x$ & $x$ \\
\hline Neophylax & $x$ & $x$ & $x$ & $x$ & $x$ & $x$ & $x$ & $x$ & $x$ & $x$ & $x$ & $x$ & & & $x$ & $x$ \\
\hline Hesperophylax & & & $x$ & & & & & & $x$ & $x$ & $x$ & $x$ & $x$ & $x$ & & $x$ \\
\hline Apatania & & & $x$ & $x$ & & $x$ & & $x$ & $x$ & $x$ & & $x$ & & & $x$ & \\
\hline Dicosmoecus & & $x$ & $x$ & & & & $x$ & & & & $x$ & $x$ & $x$ & & $x$ & $x$ \\
\hline Ecclisomyia & $x$ & $x$ & $x$ & & $x$ & $x$ & $x$ & $x$ & $x$ & $x$ & $x$ & $x$ & & $x$ & $x$ & \\
\hline Lenarchus & & & $x$ & & & & & & & $x$ & $x$ & & & & & \\
\hline Chryanda & $x$ & & & & & & & & & & & & & & & \\
\hline Onocosmoecus & $x$ & $x$ & $x$ & $x$ & $x$ & $x$ & $x$ & & $x$ & $x$ & $x$ & $x$ & & & $x$ & $x$ \\
\hline Clostoeca & & & & & & & & $x$ & & & & & & & & \\
\hline Goera & $x$ & $x$ & $x$ & $x$ & & & & & & & & & & & & \\
\hline Hydatophylax & $x$ & & & & & & & $x$ & & & & & & & & \\
\hline Cryptochia & $x$ & & & & & & $x$ & $x$ & & & & $x$ & & & & \\
\hline Psychoglypha & & & & & & & & & $x$ & & & $x$ & & & & \\
\hline Neothremma & & & & & $x$ & & $x$ & & & & & & & & & \\
\hline
\end{tabular}

Table continued on next page. 


\begin{tabular}{|c|c|c|c|c|c|c|c|c|c|c|c|c|c|c|c|c|}
\hline \multirow[b]{2}{*}{ Taxa } & \multicolumn{4}{|c|}{ Coastal } & \multicolumn{4}{|c|}{ Cascade } & \multicolumn{4}{|c|}{ Central } & \multicolumn{4}{|c|}{ Eastern } \\
\hline & $\frac{\grave{D}}{\grave{3}}$ & 号 & $\begin{array}{l}\text { ¿े } \\
\text { है } \\
\text { ஸे }\end{array}$ & $\overline{\bar{\pi}}$ & $\frac{\grave{d}}{\grave{c}}$ & 은 & $\begin{array}{l}\stackrel{\bar{d}}{E} \\
\text { है } \\
\text { ڤ }\end{array}$ & 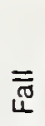 & $\stackrel{\stackrel{\Phi}{\leftrightarrows}}{\vdots}$ & 号 & 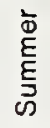 & $\overline{\bar{\pi}}$ & $\frac{\stackrel{\frac{1}{\Phi}}{\leftrightarrows}}{3}$ & 号 & $\begin{array}{l}\stackrel{\Phi}{E} \\
\stackrel{E}{\Xi}\end{array}$ & $\overline{\bar{\varpi}}$ \\
\hline $\begin{array}{l}\text { Limnephilus } \\
\text { Grammotaulius }\end{array}$ & & & & & - & $x$ & & & $x$ & & $x$ & $x$ & & & & \\
\hline Philopotamidae & & $x$ & $x$ & $x$ & $x$ & $x$ & $x$ & $x$ & & & $x$ & $x$ & $x$ & & $x$ & $x$ \\
\hline $\begin{array}{l}\text { Wormaldia } \\
\text { Dolophilodes }\end{array}$ & $x$ & $x$ & $x$ & $x$ & & $\frac{x}{x}$ & $\begin{array}{l}x \\
x\end{array}$ & & & & $x$ & & & & $x$ & \\
\hline Rhyacophilidae & $x$ & $x$ & $x$ & $x$ & $x$ & $x$ & $x$ & $x$ & $x$ & $x$ & $x$ & $x$ & $x$ & $x$ & $x$ & $x$ \\
\hline Rhyacophila & $x$ & $x$ & $x$ & $x$ & $x$ & $x$ & $x$ & $x$ & $x$ & & $x$ & $x$ & $x$ & $x$ & $x$ & \\
\hline R. acropedes & & & & $x$ & $x$ & $x$ & $x$ & $x$ & & & & & & & & \\
\hline R. angelita & & $x$ & & & & $x$ & & & & & & & & $x$ & & \\
\hline R. tucula & & & & & & & $x$ & & & & & & & & & \\
\hline R. blarina & & & & $x$ & $x$ & & $x$ & & & & & & & & & \\
\hline R. narvae & & & & & $x$ & $x$ & $x$ & $x$ & & & & & & & & \\
\hline R. vaefes & & & & & $x$ & $x$ & $x$ & $x$ & & & & & & $x$ & & \\
\hline R. betteni group & & & 1 & $x$ & & $x$ & $x$ & $x$ & & & & & & & & \\
\hline R. verrula & & $x$ & & & $x$ & $x$ & $x$ & & & & & & & & & \\
\hline R. arnaudi:" & & & & $x$ & & & & & & & & & & & & \\
\hline $\begin{array}{l}\text { Himalopsyche } \\
\text { H. phryganea }\end{array}$ & & & & & & & & $x$ & & & & & & & & $x$ \\
\hline Hydropsychidae & $x$ & $x$ & $x$ & $x$ & $x$ & $x$ & $x$ & $x$ & $x$ & $x$ & $x$ & $x$ & $x$ & $x$ & $x$ & $x$ \\
\hline Hydropsyche & & $x$ & $x$ & $x$ & & & & & $x$ & $x$ & $x$ & $x$ & $x$ & $x$ & $x$ & $x$ \\
\hline Arctopsyche & & & $x$ & $x$ & $x$ & $x$ & $x$ & $x$ & $x$ & $x$ & $x$ & $x$ & & & & $x$ \\
\hline Parapsyche & & $x$ & $x$ & $x$ & $x$ & $x$ & $x$ & $x$ & $\dot{x}$ & $x$ & $x$ & $x$ & & & & \\
\hline Homoplectra & & & & & $x$ & & & & & & & & & & & \\
\hline Cheumatopsyche & $x$ & $x$ & & $x$ & & & & & & & & & $x$ & $x$ & $x$ & $x$ \\
\hline Psychomyiidae & & & $i$ & & $x$ & $x$ & $x$ & & & & $x$ & $x$ & & & & \\
\hline Psychomyia & & $x$ & $x$ & $x$ & $x$ & & & & & & & & & & $x$ & $x$ \\
\hline Polycentropodidae & & & $x$ & & $x$ & $x$ & $x$ & $x$ & & $x$ & & $x$ & & & $x$ & \\
\hline Polycentropus & & & $x$ & & & $x$ & $x$ & $x$ & & & & & & & & \\
\hline Brachycentridae & $x$ & $x$ & $x$ & $x$ & $x$ & $x$ & $x$ & $x$ & $x$ & $x$ & $x$ & $x$ & & & & \\
\hline Micrasema & $x$ & $x$ & & $x$ & $x$ & $x$ & $x$ & $x$ & $x$ & $x$ & $x$ & $x$ & & & & \\
\hline Calamoceratidae & & & & & & $x$ & & & & & & & & & & \\
\hline Heteropleciron & & & & $x$ & & & & $x$ & & & & & & & & \\
\hline Lepidostomatidae & & & & & & & & & & & & & & $x$ & $x$ & $x$ \\
\hline $\begin{array}{l}\text { Lepidostoma } \\
\text { Glossosomatidae }\end{array}$ & $\begin{array}{l}x \\
x\end{array}$ & $\begin{array}{l}x \\
x\end{array}$ & $\begin{array}{l}x \\
x\end{array}$ & $\begin{array}{l}x \\
x\end{array}$ & $\begin{array}{l}x \\
x\end{array}$ & $\begin{array}{l}x \\
x\end{array}$ & $\begin{array}{l}x \\
x\end{array}$ & $\begin{array}{l}x \\
x\end{array}$ & $\begin{array}{l}x \\
x\end{array}$ & $\begin{array}{l}x \\
x\end{array}$ & $\begin{array}{l}x \\
x\end{array}$ & $\begin{array}{l}x \\
x\end{array}$ & $\begin{array}{l}x \\
x\end{array}$ & $\begin{array}{l}x \\
x\end{array}$ & $\frac{x}{x}$ & $\dot{x}$ \\
\hline Anagapetus & $\hat{x}$ & $\alpha$ & $\hat{x}$ & $n$ & $\hat{x}$ & $\hat{x}$ & & $x$ & & & $x$ & $x$ & & & & $x$ \\
\hline Agapetus & & & & & $x$ & & & & & & $x$ & $x$ & & & $x$ & \\
\hline Glossosoma & $x$ & $x$ & $x$ & $x$ & & $x$ & $x$ & & & & & $x$ & $x$ & & & $x$ \\
\hline Phryganeidae & & & $x$ & & & & $x$ & & & & & $x$ & & & & \\
\hline Hydroptilidae & $x$ & & $x$ & $x$ & & & $x$ & $x$ & & & & & & & $x$ & $x$ \\
\hline Hydroptila & & & & & & & & & & & & & $x$ & & $x$ & $x$ \\
\hline Palaeagapetus & & & & & & & $x$ & & & & & & & & & \\
\hline Ochrotrichia & & & & & & & & $x$ & & & & & & & & \\
\hline Leptoceridae & & & & & & & & & & & & & & & $x$ & \\
\hline Lepidoptera & & $x$ & $x$ & $x$ & & $x$ & $x$ & $x$ & & $x$ & $x$ & $x$ & & $x$ & $x$ & $x$ \\
\hline Pieridae & & & & & & & $x$ & & & & & & & & & \\
\hline Lycaenidae & & & & $x$ & & & $x$ & & & & & & & & & \\
\hline Satyridae & & & & $x$ & & & & & & & & $x$ & & & & $x$ \\
\hline Hesperiidae & & & & $x$ & & & $x$ & & & & & $x$ & & & & $x$ \\
\hline Arctiidae & & & & & & & & & & & $x$ & $x$ & & & & \\
\hline Noctuidae & & & & & & & & & & $x$ & $x$ & $x$ & & & $x$ & $x$ \\
\hline Geometridae & & $x$ & $x$ & $x$ & & & $x$ & $x$ & & & $x$ & $x$ & & & $x$ & \\
\hline Microlepidoptera & & $x$ & $x$ & & & & $x$ & & & $x$ & $x$ & $x$ & & & $x$ & $x$ \\
\hline Pyralidae & & & & $x$ & & & $x$ & & & & & & & $x$ & & $x$ \\
\hline
\end{tabular}

Table continued on next page. 


\begin{tabular}{|c|c|c|c|c|c|c|c|c|c|c|c|c|c|c|c|c|}
\hline \multirow[b]{2}{*}{ Taxa } & \multicolumn{4}{|c|}{ Coastal } & \multicolumn{4}{|c|}{ Cascade } & \multicolumn{4}{|c|}{ Central } & \multicolumn{4}{|c|}{ Eastern } \\
\hline & 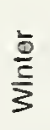 & $\frac{\text { D }}{\frac{D}{2}}$ & 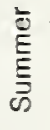 & $\overline{\overline{10}}$ & $\frac{\frac{2}{\varrho}}{\frac{5}{3}}$ & 号 & 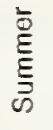 & $\overline{\bar{W}}$ & $\frac{\grave{\Phi}}{\sum}$ & $\frac{\mathscr{E}}{\frac{5}{2}}$ & 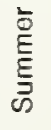 & $\overline{\bar{\varpi}}$ & $\frac{\grave{D}}{\frac{5}{5}}$ & $\frac{\mathscr{D}}{\frac{5}{c}}$ & 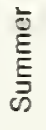 & $\overline{\bar{\pi}}$ \\
\hline Aegeriidae (Sesiidae) & & & & & & & $x$ & & & & & & & & & \\
\hline Diptera & $x$ & $x$ & $x$ & $x$ & $x$ & $x$ & $x$ & $x$ & $x$ & $x$ & $x$ & $x$ & & $x$ & $x$ & $x$ \\
\hline Nematocera & $x$ & $x$ & & $x$ & $x$ & & & $x$ & & $x$ & $x$ & $x$ & & $x$ & $x$ & $x$ \\
\hline Tanyderidae & & $x$ & & $x$ & & & $x$ & $x$ & & & & & & & & \\
\hline Tipulidae & $x$ & $x$ & $x$ & $x$ & $x$ & $x$ & $x$ & $x$ & $x$ & $x$ & $x$ & $x$ & $x$ & $x$ & $x$ & $x$ \\
\hline Limoniinae & & & & & & & & & & & & & & $x$ & & \\
\hline Antocha & $x$ & $x$ & $x$ & $x$ & $x$ & $x$ & $x$ & $x$ & $x$ & $x$ & $x$ & $x$ & $x$ & $x$ & $x$ & $x$ \\
\hline Limnophila & & $x$ & $x$ & & $x$ & $x$ & $x$ & & & & $x$ & & & $x$ & $x$ & \\
\hline Dicranota & $x$ & $x$ & $x$ & $x$ & $x$ & $x$ & $x$ & $x$ & $x$ & $x$ & $x$ & $x$ & $x$ & $x$ & $x$ & $x$ \\
\hline Tipula & & & $x$ & & $x$ & & & $x$ & & & & & & & & $x$ \\
\hline Hexatoma & $x$ & $x$ & $x$ & $x$ & $x$ & $x$ & $x$ & $x$ & $x$ & $x$ & $x$ & $x$ & $x$ & $x$ & $x$ & $x$ \\
\hline Hesperoconopa & $x$ & $x$ & $x$ & $x$ & $x$ & & & $x$ & $x$ & & & & & & $x$ & \\
\hline Molophilus & $x$ & $x$ & $x$ & $x$ & & & & & & & & & & & & \\
\hline Psychodidae & $x$ & $x$ & $x$ & $x$ & $x$ & $x$ & $x$ & $x$ & $x$ & $x$ & $x$ & $x$ & $x$ & $x$ & $x$ & $x$ \\
\hline Psychoda & & & & & & & & & & & & & $x$ & & & \\
\hline Pericoma & & $x$ & & $x$ & $x$ & & & & & $x$ & & & & & & $x$ \\
\hline Maruina & & & & & & & $x$ & & & & & & & & $x$ & \\
\hline Ptychopteridae & $x$ & $x$ & & $x$ & & & & & & & & & & & & \\
\hline Blephariceridae & & & & & $x$ & $x$ & $x$ & & & $x$ & $x$ & $x$ & & $x$ & & $x$ \\
\hline Deuterophlebijdae & & & & & & $x$ & & & & & & & & & & \\
\hline Dixidae & $x$ & $x$ & $x$ & $x$ & $x$ & $x$ & $x$ & $x$ & & $x$ & $x$ & $x$ & & $x$ & $x$ & $x$ \\
\hline Meringodixa & & & & $x$ & & & $x$ & & & & $x$ & & & & $x$ & $x$ \\
\hline Dixa & & $x$ & $x$ & $x$ & & & $x$ & $x$ & & & $x$ & & & & $x$ & $x$ \\
\hline Culicidae & & $x$ & & $x$ & & & $x$ & & & & & $x$ & & & & \\
\hline Ceratopogonidae & $x$ & $x$ & $x$ & $x$ & $x$ & $x$ & $x$ & $x$ & $x$ & $x$ & $x$ & $x$ & & $x$ & $x$ & $x$ \\
\hline $\begin{array}{l}\text { Forcipom;ia } \\
\text { Bezzia }\end{array}$ & & & $x$ & & & & $x$ & $x$ & & & & & & & & \\
\hline Chironomidae & $x$ & $x$ & $x$ & $x$ & $x$ & $x$ & $x$ & $x$ & $x$ & $x$ & $x$ & $x$ & $x$ & $x$ & $x$ & $x$ \\
\hline Simuliidae & $x$ & $x$ & $x$ & $x$ & $x$ & $x$ & $x$ & $x$ & $x$ & $x$ & $x$ & $x$ & $x$ & $x$ & $x$ & $x$ \\
\hline Bibionidae & & $x$ & & & & $x$ & & & & & & $x$ & & & & $x$ \\
\hline Mycetophilidae & $x$ & $x$ & $x$ & $x$ & $x$ & $x$ & $x$ & $x$ & & $x$ & $x$ & $x$ & & $x$ & $x$ & $x$ \\
\hline Sciaridae & $x$ & $x$ & $x$ & $x$ & & $x$ & $x$ & $x$ & & $x$ & $x$ & $x$ & & $x$ & $x$ & $x$ \\
\hline Cecidomyiidae & & $x$ & $x$ & $x$ & & & $x$ & $x$ & & $x$ & $x$ & $x$ & & $x$ & $x$ & $x$ \\
\hline Brachycera & & & & & & & & & & & & & & & & $x$ \\
\hline Xylomyidae & & & & & & & & & & & $x$ & & & & & \\
\hline Stratiomyidae & & & & $x$ & & & & $x$ & $x$ & & $x$ & $x$ & & $x$ & & \\
\hline Tabanidae & $x$ & & $x$ & & & & $x$ & & $x$ & $x$ & $x$ & $x$ & $x$ & $x$ & $x$ & $x$ \\
\hline Rhagionidae & & $x$ & $x$ & & & & $x$ & & & & $x$ & & & & $x$ & \\
\hline Therevidae & & & $x$ & & & & & & & & $x$ & $x$ & & & & \\
\hline Periscelididae & & & & & & & $x$ & & & & & & & & $x$ & \\
\hline Asilidae & & $x$ & $x$ & & & & $x$ & & & & $x$ & $x$ & & & $x$ & \\
\hline Acroceridae & & & $x$ & & & & $x$ & & & & $x$ & & & & $x$ & \\
\hline Bombyliidae & & & & & & & $x$ & & & & & & & & $x$ & \\
\hline Empididae & $x$ & $x$ & $x$ & $x$ & $x$ & $x$ & $x$ & $x$ & $x$ & $x$ & $x$ & $x$ & & $x$ & $x$ & $x$ \\
\hline Dolichopodidae & $x$ & $x$ & $x$ & $x$ & $x$ & $x$ & $x$ & $x$ & & $x$ & $x$ & $x$ & & $x$ & $x$ & $x$ \\
\hline Cyclormapha & & & & & & & & & & & $x$ & & & & $x$ & $x$ \\
\hline Lonchopteridae & & & $x$ & $x$ & & & $x$ & & & & $x$ & $x$ & & & $x$ & $x$ \\
\hline Phoridae & $x$ & $x$ & $x$ & $x$ & $x$ & $x$ & $x$ & $x$ & & $x$ & $x$ & $x$ & & $x$ & $x$ & $x$ \\
\hline Pipunculidae & & & $x$ & $x$ & & & $x$ & & & & $x$ & $x$ & & & & \\
\hline $\begin{array}{l}\text { Syrphidae } \\
\text { Conopidae }\end{array}$ & & $x$ & $x$ & $x$ & & & $\begin{array}{l}x \\
x\end{array}$ & $x$ & & & $x$ & $x$ & & & $x$ & \\
\hline
\end{tabular}

Table continued on next page. 


\begin{tabular}{|c|c|c|c|c|c|c|c|c|c|c|c|c|c|c|c|c|}
\hline \multirow[b]{2}{*}{ Taxa } & \multicolumn{4}{|c|}{ Coastal } & \multicolumn{4}{|c|}{ Cascade } & \multicolumn{4}{|c|}{ Central } & \multicolumn{4}{|c|}{ Eastern } \\
\hline & $\stackrel{\stackrel{\Phi}{\leftrightarrows}}{\vdots}$ & $\begin{array}{l}\text { ㅁ } \\
\text { की }\end{array}$ & $\begin{array}{l}\stackrel{\bar{\Xi}}{E} \\
\text { 心 }\end{array}$ & $\overline{\bar{\varpi}}$ & 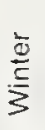 & $\frac{\sigma}{\frac{5}{2}}$ & 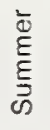 & $\overline{\bar{\pi}}$ & $\frac{\frac{2}{\Xi}}{3}$ & $\frac{\text { D }}{\frac{1}{2}}$ & $\stackrel{\Xi}{E}$ & $\overline{\bar{w}}$ & $\frac{\grave{\Xi}}{3}$ & $\begin{array}{l}\frac{D}{5} \\
\frac{5}{5} \\
\text { की }\end{array}$ & 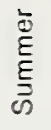 & $\overline{\bar{\sigma}}$ \\
\hline Sepsidae & & & & & & & $x$ & & & $x$ & $x$ & $x$ & & & & \\
\hline Sciomyzidae & & & & $x$ & & & $x$ & & & & & & & & $x$ & \\
\hline Lauxaniidae & & & $x$ & & & $x$ & $x$ & & & & & & & & $x$ & \\
\hline Lonchaeidae & & $x$ & & & & $x$ & & & & $x$ & & & & $x$ & & \\
\hline Sphaeroceridae & $x$ & $x$ & $x$ & $x$ & & & $x$ & $x$ & & $x$ & & $x$ & & $\therefore$ & $x$ & \\
\hline Milichiidae & & & $x$ & & & & $x$ & & & & & & & & $x$ & $x$ \\
\hline Tephritidae & & $x$ & & & & & $x$ & $x$ & & & & & & & & \\
\hline Ephydridae & $x$ & $x$ & $x$ & $x$ & $x$ & $x$ & $x$ & $x$ & & $x$ & $x$ & $x$ & & $x$ & $x$ & $x$ \\
\hline Drosophilidae & & $x$ & $x$ & $x$ & & $x$ & $x$ & $x$ & & & $x$ & $x$ & & & $x$ & $x$ \\
\hline Chloropidae & & $x$ & $x$ & $x$ & & $x$ & $x$ & & & & $x$ & $x$ & & & $x$ & \\
\hline Agromyzidae & & $x$ & $x$ & $x$ & & & $x$ & & & & $x$ & $x$ & & & $x$ & $x$ \\
\hline Clusiidae & $x$ & $x$ & $x$ & & & & & & & & & & & & $x$ & \\
\hline Heleomyzidae & & & $x$ & & & $x$ & & & & & & $x$ & & & $x$ & $x$ \\
\hline Anthomyzidae & & & $x$ & & & & & & & & $x$ & & & & $x$ & \\
\hline Anthomyiidae & & & & & & & & & & & & & & & & \\
\hline (Scatophagidae) & & $x$ & $x$ & $x$ & & & $x$ & $x$ & & $x$ & $x$ & $\mathrm{x}$ & & $x$ & $x$ & $x$ \\
\hline Muscoidea & $x$ & $x$ & $x$ & $x$ & $x$ & $x$ & $x$ & $x$ & & $x$ & $x$ & $x$ & & $x$ & $x$ & $x$ \\
\hline Muscidae & & $x$ & $x$ & $x$ & & $x$ & $x$ & $x$ & & & $x$ & $x$ & & $x$ & $x$ & $x$ \\
\hline Oestroidea & & & & & & & & & & & $x$ & & & & & \\
\hline Calliphoridae & & & $x$ & & & $x$ & $x$ & & & $x$ & $x$ & $x$ & & & $x$ & $x$ \\
\hline Sarcophagidae & & $x$ & $x$ & & & & $x$ & & & & $x$ & & & & $x$ & \\
\hline Tachinidae & & $x$ & $x$ & $x$ & & & $x$ & $x$ & & $x$ & $x$ & $x$ & & & $x$ & $x$ \\
\hline Siphonaptera & & & & & & & $x$ & & & & & & & & & \\
\hline Hymenoptera & & $x$ & $x$ & $x$ & & & $x$ & $x$ & & $x$ & $x$ & $x$ & & $x$ & $x$ & $x$ \\
\hline Symphyta & & & & & & & & & & & $x$ & & & & & \\
\hline Cimbicidae & & & $x$ & & & & & & & & & & & & & \\
\hline Tenthredinidae & & & $x$ & & & & & & & & & & & & & \\
\hline Apocrita & & & & & & & & & & & & & & & & \\
\hline Braconidae & & & $x$ & $x$ & & & $x$ & $x$ & & $x$ & $x$ & $x$ & & & $x$ & $x$ \\
\hline Ichneumonidae & & $x$ & $x$ & $x$ & & $x$ & $x$ & $x$ & & $x$ & $x$ & $x$ & & & $x$ & $x$ \\
\hline Chalcidoidea & & $x$ & $x$ & $x$ & & $x$ & $x$ & $x$ & & $x$ & $x$ & $x$ & & & $x$ & $x$ \\
\hline Mymaridae & & & & & & & & $x$ & & & $x$ & $x$ & & & $x$ & $x$ \\
\hline Eulophidae & & $x$ & & & & & & & & & & & & & & \\
\hline Pteromalidae & & & & & & & & & & & $x$ & $x$ & & & & \\
\hline Cynipoidea & & & & $x$ & & & & & & & $x$ & & $x$ & & $x$ & $x$ \\
\hline $\begin{array}{l}\text { Cynipidae } \\
\text { Evaniidae }\end{array}$ & & $x$ & & $x$ & & & $x$ & $x$ & & & $x$ & $x$ & & & $x$ & $x$ \\
\hline Proctotrupoidea & & $x$ & $x$ & $x$ & & $x$ & $x$ & $x$ & & $x$ & $\hat{x}$ & $x$ & & & $x$ & $x$ \\
\hline Proctotrupidae & & & $x$ & & & & & & & $x$ & $x$ & & & & & \\
\hline Diapriidae & & & & $x$ & & & $x$ & $x$ & & & $x$ & $x$ & & & $x$ & \\
\hline Platygasteridae & & & & $x$ & & & & & & & $x$ & $x$ & & & & $x$ \\
\hline Bethyloidea & & & & $x$ & & & & & & & & & & & & \\
\hline Dryinidae & & & & & & & & & & & $x$ & & & & & \\
\hline Formicidae & $x$ & $x$ & $x$ & $x$ & & $x$ & $x$ & $x$ & & $x$ & $x$ & $x$ & & $x$ & $x$ & $x$ \\
\hline Vespoidea & & & & & & & & & & & & & & & & \\
\hline $\begin{array}{l}\text { Vespidae } \\
\text { Pompilidae }\end{array}$ & & & & & & & $x$ & $x$ & & & $x$ & $x$ & & & & \\
\hline Sphecidae & & & & $x$ & & & $x$ & $x$ & & & $x$ & $x$ & & & $x$ & $x$ \\
\hline Apoidea & & $x$ & $x$ & & & & $x$ & $x$ & & & & & & & $x$ & \\
\hline Halictidae & & & & & & & & & & & & $x$ & & & $x$ & $x$ \\
\hline Apidae & & $x$ & & & & & & $x$ & & & $x$ & $x$ & & & $x$ & \\
\hline Chilopoda & & $x$ & $x$ & $x$ & & & & & & & & & & & & \\
\hline
\end{tabular}

Table continued on next page. 


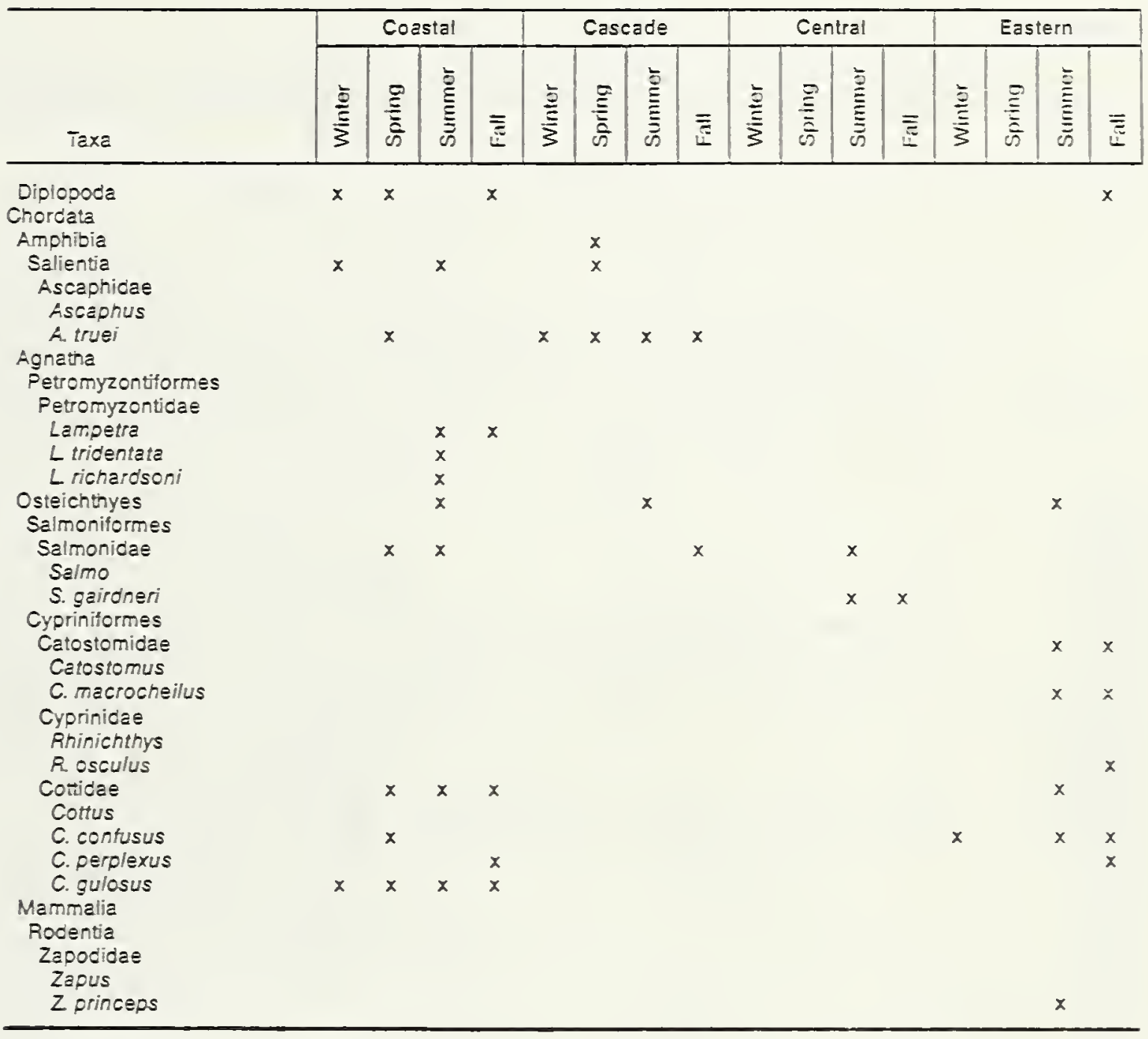




\begin{tabular}{|c|c|c|c|c|c|c|c|c|c|c|c|c|c|c|c|c|}
\hline \multirow[b]{2}{*}{ Taxa } & \multicolumn{4}{|c|}{ Benthic } & \multicolumn{4}{|c|}{ Drift } & \multicolumn{4}{|c|}{ Sticky } & \multicolumn{4}{|c|}{ Water } \\
\hline & Winter & Spring & Summer & Fall & Winter & Spring & Summer & Fall & Winter & Spring & Summer & Fall & Winter & Spring & Sernmer & Fall \\
\hline \multicolumn{17}{|c|}{ Percent } \\
\hline $\begin{array}{l}\text { Plecoptera } \\
\text { Pteronarcidae }\end{array}$ & & 9.7 & 11.6 & 4.3 & & $\begin{array}{r}13.3 \\
6.7\end{array}$ & $\begin{array}{l}22.6 \\
19.4\end{array}$ & 35.0 & 25.0 & 33.3 & 12.5 & 26.1 & & & & $\begin{array}{r}5.9 \\
11.8\end{array}$ \\
\hline Peltoperlidae & & & 2.3 & 2.1 & & & & 5.0 & & & & & & & & \\
\hline Taeniopterygidae & 37.5 & & 4.7 & 6.4 & 9.1 & 6.7 & & & & & & & & & & \\
\hline Nemouridae & 29.2 & 25.8 & 32.6 & 21.3 & 27.3 & 66.7 & 38.7 & 50.0 & 31.3 & 53.3 & 12.5 & 30.4 & 57.1 & 33.3 & 21.4 & \\
\hline Leuctridae & 12.5 & 6.5 & 16.3 & 4.3 & & 20.0 & 6.5 & 30.0 & & & & & & 44.4 & & \\
\hline Capniidae & 37.5 & 19.4 & 11.6 & 29.8 & 18.2 & & 6.5 & 750 & & & & 4.3 & 14.3 & 11.1 & & 23.5 \\
\hline Perlidae & 16.7 & 54.8 & 83.7 & 72.3 & & 6.7 & 25.8 & 5.0 & & & & & & & 7.1 & \\
\hline Perlodidae & 50.0 & 64.5 & 41.9 & 40.4 & 9.1 & 33.3 & 290 & 25.0 & & & & & & & 7.1 & \\
\hline Chloroperlidae & 54.2 & 100.0 & 97.7 & 93.6 & 18.2 & 33.3 & 38.7 & 50.0 & & 13.3 & 16.7 & & & 222 & 35.7 & \\
\hline Psocoptera & & & 2.3 & & & & 6.5 & 5.0 & & & & & & & $\begin{array}{r}28.6 \\
7.1\end{array}$ & 41.2 \\
\hline $\begin{array}{l}\text { Mallophaga } \\
\text { Thysanoptera }\end{array}$ & & & & & & & & & & & & & & 33.3 & $\begin{array}{r}7.1 \\
14.3\end{array}$ & 5.9 \\
\hline & & & 2.3 & & & 6.7 & 12.9 & 20.0 & & 6.7 & 4.2 & 13.0 & & 11.1 & 42.9 & 3.9 \\
\hline $\begin{array}{l}\text { Hemiptera } \\
\text { Macroveliidae }\end{array}$ & & & & & & & & & & 0.8 & 4.2 & & & & & 5.9 \\
\hline Gerridae & & & & 2.1 & & 6.7 & 6.5 & 10.0 & & 20.0 & 16.7 & 130 & 28.6 & 77.8 & 129 & 52.9 \\
\hline Velijdae & & & & & & & 3.2 & 5.0 & & 20.0 & 4.2 & 21.7 & & 33.3 & 7.1 & 11.8 \\
\hline Mesoveliidae & & & & & & & & & & 13.3 & & & & & & \\
\hline Saldidae & & & & & & & & & & 13.3 & 41.7 & & & & 57.1 & \\
\hline Miridae & & & & 2.1 & & & & & & & & & & 11.1 & 14.3 & \\
\hline Tingidae & & & & & & 13.3 & & & & 13.3 & & & & 11.1 & 7.1 & 5.9 \\
\hline Aladidae & & & & & & & 3.2 & & & & & & & & & \\
\hline $\begin{array}{l}\text { Lygaeidae } \\
\text { Pentatomidae }\end{array}$ & & & & & & 13.3 & $\begin{array}{l}3.2 \\
32\end{array}$ & & & 13.3 & 4.2 & 4.3 & & & 14.3 & 59 \\
\hline Homoptera & & & & & & & $\begin{array}{l}3.2 \\
9.7\end{array}$ & 10.0 & & 6.7 & 8.3 & 8.7 & 14.3 & & 35.7 & 23.5 \\
\hline Cicadellidae & & & 4.7 & 43 & 9.1 & & & 15.0 & 6.3 & 20.0 & 37.5 & 26.1 & 14.3 & & 78.6 & 29.4 \\
\hline Cercopidae & & & 2.3 & & & & & 10.0 & & & & 13.0 & & & 7.1 & 52.9 \\
\hline Psyllidae & & & & & & & & & & 13.3 & & 4.3 & & & 28.6 & 11.8 \\
\hline Aphididae & & & 2.3 & & & 6.7 & 9.7 & 45.0 & & 33.3 & 12.5 & 34.8 & 14.3 & 22.2 & 78.6 & 64.7 \\
\hline $\begin{array}{l}\text { Eriosomatidae } \\
\text { Coccoidea }\end{array}$ & & & & & & 6.7 & 3.2 & & & & & & & & & 5.9 \\
\hline Chermidae & & & & & & & & & & & & & & & & 5.9 \\
\hline Coleoptera & 4.2 & 3.2 & 4.7 & 2.1 & 9.1 & 13.3 & 6.5 & 5.0 & 12.5 & 53.3 & 37.5 & 13.0 & & 11.1 & 57.1 & 5.9 \\
\hline $\begin{array}{l}\text { Lathridiidae } \\
\text { Carabidae }\end{array}$ & & & & & & & & & & 6.7 & & & & 11.1 & 7.1 & $\begin{array}{r}11.8 \\
5.9\end{array}$ \\
\hline Amphizoidae & & & & & & & 3.2 & 10.0 & & & & & & & & \\
\hline Dytiscidae & & & 30.2 & 12.8 & 9.1 & 33.3 & 16.1 & & & 6.7 & 4.2 & 4.3 & & & & \\
\hline $\begin{array}{l}\text { Melyridae } \\
\text { Gyrinidae }\end{array}$ & & & & & & & & & & & & & 14.3 & & 7.1 & \\
\hline $\begin{array}{l}\text { Gyrınidae } \\
\text { Histeridae }\end{array}$ & & & & & & & & & & & & & & & 7.1 & \\
\hline Hydrophilidae & & & 4.7 & & & 13.3 & 6.5 & 20.0 & & 13.3 & 16.7 & 4.3 & & 22.2 & 42.9 & 5.9 \\
\hline $\begin{array}{l}\text { Hydraenidae } \\
\text { (Limnebiidae) }\end{array}$ & & & & & & & & & & & & & & 222 & 7.1 & \\
\hline Ptiliidae & & & & & & & & & & 13.3 & 4.2 & 4.3 & & 11.1 & 28.6 & 11.8 \\
\hline Chrysomelidae & & & & 2.1 & & & 22.6 & & & 33.3 & 20.8 & & 28.6 & 33.3 & 57.1 & \\
\hline Staphylinidae & & & & & & 26.7 & 9.7 & 10.0 & 31.3 & 60.0 & 54.2 & 13.0 & 28.6 & 88.9 & 85.7 & 29.4 \\
\hline Colydiidae & & & & & & & 3.2 & 5.0 & & & & & & & & \\
\hline $\begin{array}{l}\text { Cantharidae } \\
\text { Curculionidae }\end{array}$ & & & & & & 6.7 & & & & 13.3 & 4.2 & & & 44.4 & & \\
\hline (Nemonychidae) & & & & & & & & & & 6.7 & & 4.3 & & & 7.1 & \\
\hline Elateridae & & & & & & & 3.2 & & & & & 4.3 & & & 14.3 & \\
\hline Byrrhidae & & & & & & & & & 6.3 & & & 8.7 & & & & 11.8 \\
\hline Dascillidae & & & & & & & & & & & & & & & 14.3 & \\
\hline Elmidae & 50.0 & 96.8 & 100.0 & 100.0 & 27.3 & 53.3 & 67.7 & 75.0 & & & 16.7 & & & 11.1 & 35.7 & 17.6 \\
\hline Scolytidae & & & & & & & & & & 6.7 & & & & 11.1 & 28.6 & \\
\hline $\begin{array}{l}\text { Cryptophagidae } \\
\text { Nitudulidae }\end{array}$ & & 6.5 & & & & & & & & & & & & 11.1 & & \\
\hline $\begin{array}{l}\text { Nitudulidae } \\
\text { Endomychidae }\end{array}$ & 4.2 & & & & & & & & & & & & & 11.1 & 7.1 & \\
\hline Endomychidae & & & & & & & & & & & & & & & & \\
\hline
\end{tabular}

Table continued on next page. 


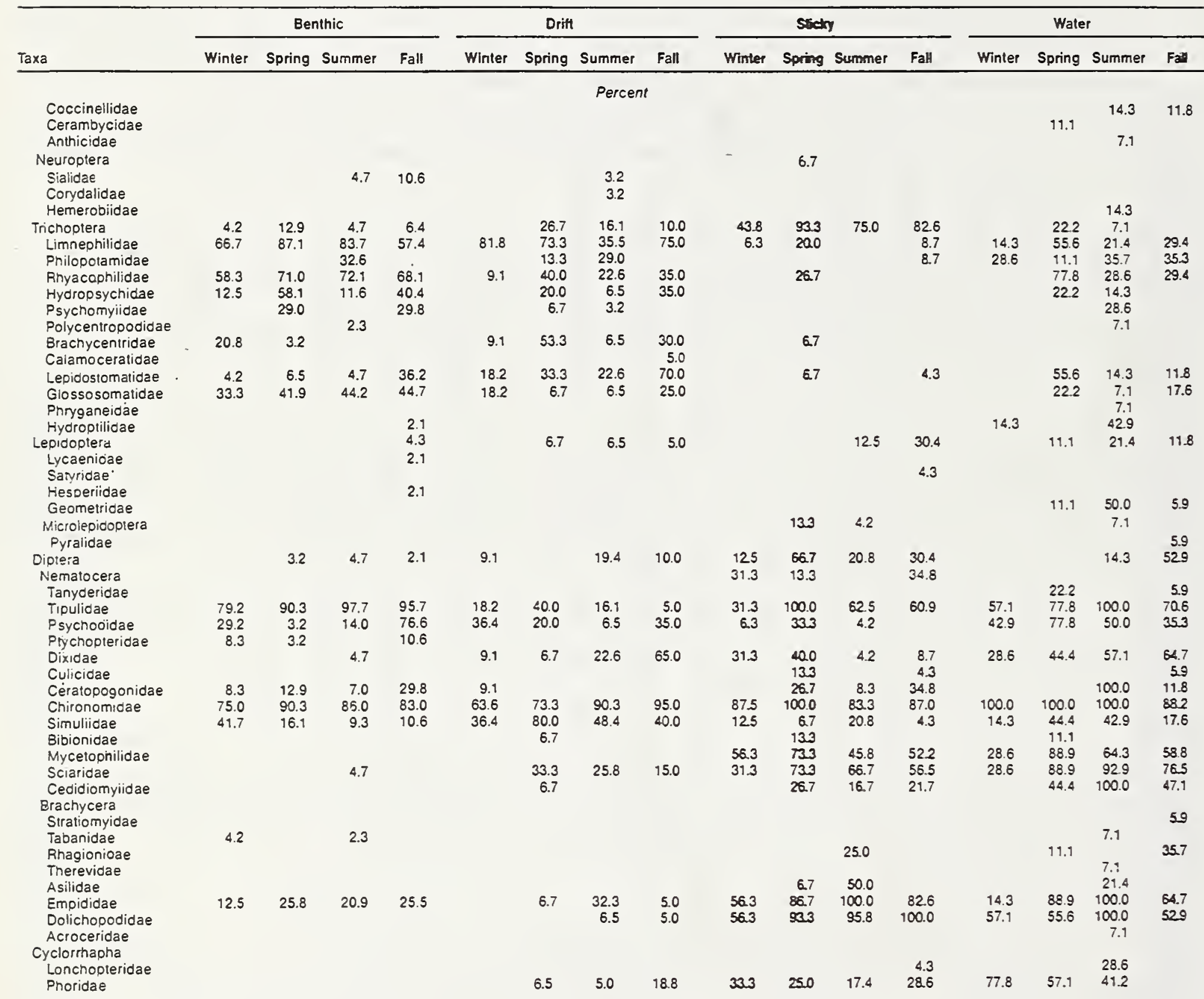

Table continued on next page. 


\begin{tabular}{|c|c|c|c|c|c|c|c|c|c|c|c|c|c|c|c|c|}
\hline \multirow[b]{2}{*}{ Taxa } & \multicolumn{4}{|c|}{ Benthic } & \multicolumn{4}{|c|}{ Drift } & \multicolumn{4}{|c|}{ Sticky } & \multicolumn{4}{|c|}{ Water } \\
\hline & Winter & Spring & Summer & Fall & Winter & Spring & Summer & Fall & Winter & Spring & Summer & Fall & Winte: & Spring & Summer & Fall \\
\hline \multicolumn{17}{|c|}{ Percent } \\
\hline Pipunculidae & & & & & & & 3.2 & & & & & & & & & 5.9 \\
\hline Syrphidae & & & & & & & & & & 400 & 12.5 & & & & 7.1 & 11.8 \\
\hline Lauxaniidae & & & & & & & & & & & & & & & 7.1 & \\
\hline Lonchaeidae & & & & & & & & & & 6.7 & & & & & & \\
\hline $\begin{array}{l}\text { Sphaeroceridae } \\
\text { Milichiidae }\end{array}$ & & & & & & & & & & 13.3 & 12.5 & & 14.3 & 22.2 & $\begin{array}{r}64.3 \\
7.1\end{array}$ & 11.8 \\
\hline Tephritidae & & & & & & & & & & & & & & 11.1 & & \\
\hline Ephydridae & & & & & & 26.7 & & & 6.3 & 20.0 & 20.8 & 39.1 & 14.3 & 55.6 & 1000 & 35.3 \\
\hline Drosophilidae & & & & & & & & & & 46.7 & & 13.0 & & 22.2 & 28.6 & \\
\hline Chloropidae & & 3.2 & & & & & & & & 6.7 & & 4.3 & & & 7.1 & \\
\hline Agromyzidae & & & & & & & & & & 13.3 & & 4.3 & & 11.1 & 7.1 & \\
\hline $\begin{array}{l}\text { Clusiidae } \\
\text { Sciomyzidae }\end{array}$ & & & & & & & & & & & & & 14.3 & 11.1 & 14.3 & \\
\hline $\begin{array}{l}\text { Sciomyzidae } \\
\text { Heleomyzidae }\end{array}$ & & & & & & & 3.2 & & & & & & & & & 5.9 \\
\hline Anthomyzidae & & & & & & & . & & & & & & & & 7.1 & \\
\hline Anthomyiidae & & & & & & & & & & & & & & & & \\
\hline (Scatophagidae) & & 3.2 & & & & & 3.2 & & & 33.3 & & & & 44.4 & 14.3 & 29.4 \\
\hline Platypezidae & & & & & & & & & 31.3 & 13.3 & & 34.8 & & & & \\
\hline Muscoidea & & & & & & & & & 37.5 & 33.3 & 87.5 & 56.5 & & & & 5.9 \\
\hline Muscidae & & & & & & & & & & 6.7 & & & & 44.4 & 50.0 & 17.6 \\
\hline Oestroidea & & & & & & & & & & & & & & & & \\
\hline Całliphoridae & & & & & & & & & & & & & & & 21.4 & \\
\hline Sarcophagidae & & & & & & & & & & & & & & $\$ 1.1$ & 21.4 & \\
\hline Tachinidae & & & & & & & & & & 13.3 & & 4.3 & & & 21.4 & \\
\hline Hymenoptera & & & & & & 6.7 & 3.2 & & & 26.7 & 4.2 & 17.4 & & 11.1 & 21.4 & 5.9 \\
\hline Symphyta & & & & & & & & & & & & & & & & \\
\hline Cimbicidae & & & & & & & 3.2 & & & & & & & & & \\
\hline Tenthredinidae & & & & & & & 3.2 & & & & & & & & 7.1 & \\
\hline \multicolumn{17}{|l|}{ Apocrita } \\
\hline Braconidae & & & & & & & 3.2 & 5.0 & & & & & & & & \\
\hline Ichneumonidae & & & & & & & 32 & & & 26.7 & 20.8 & 17.4 & & 44.4 & 28.6 & 17.6 \\
\hline Chalcidoidea & & & & & & 6.7 & & 5.0 & & 13.3 & 4.2 & 8.7 & & 44.4 & 50.0 & 47.1 \\
\hline Eulophıdae & & & & & & & & & & & & & & 11.1 & & \\
\hline Cynipoidea & & & & & & & & & & & & & & & & 5.9 \\
\hline Cynipidae & & & & & & 6.7 & & & & & & 8.7 & & & & \\
\hline Proctotrupoidea & & & & 2.1 & & & 3.2 & 5.0 & & & 12.5 & 4.3 & & 22.2 & 21.4 & 17.6 \\
\hline Proctotrupidae & & & & & & & & & & & & & & & 7.1 & \\
\hline Diapriidae & & & & & & & & & & & & 4.3 & & & & \\
\hline Platygasteridae & & & & & & & & & & & & 4.3 & & & & \\
\hline Bethyloidea & & & & & & & & & & & & 4.3 & & & & \\
\hline $\begin{array}{l}\text { Formicidae } \\
\text { Vespoidea }\end{array}$ & & & & & 9.1 & 6.7 & 6.5 & 15.0 & 6.3 & 13.3 & 4.2 & & & 11.1 & 35.7 & 11.8 \\
\hline Sphecidae & & & & 2.1 & & & & & & & & & & & & 5.9 \\
\hline Apoidea & & & & & & & 3.2 & & & & & & & 11.1 & 7.1 & \\
\hline Apidae & & & & & & & & & & & & & & 11.1 & & \\
\hline Chilopoda & & & & & & & & 10.0 & & & & & & 33.3 & 7.1 & 11.8 \\
\hline Diplopoda & & & & & 9.1 & & & & & & & 4.3 & & 11.1 & & 29.4 \\
\hline \multicolumn{17}{|l|}{ Chordata } \\
\hline Amphibia & & & & & & & & & & & & & & & & \\
\hline Salientia & 4.2 & & 2.3 & & & & & & & & & & & & & \\
\hline Ascaphidae & & 3.2 & & & & & & & & & & & & & & \\
\hline \multicolumn{17}{|l|}{ Agnatha } \\
\hline \multicolumn{17}{|l|}{ Petromyzontiformes } \\
\hline Petromyzontidae & & & & 2.1 & & & 29.0 & & & & & & & & & \\
\hline Osteichthyes & & & & & & & 3.2 & & & & & & & & & \\
\hline Salmoniformes & & & & & & & & & & & & & & & & \\
\hline Salmonidae & & & & & & 6.7 & 3.2 & & & & & & & & & \\
\hline Cypriniformes & & & & & & & & & & & & & & & & \\
\hline Cottidae & 12.5 & 19.4 & 27.9 & 25.5 & 9.1 & & 35.5 & 20.0 & & & & & & & & \\
\hline
\end{tabular}

Table continued on next page. 


\section{Percentage of Samples From Cascade Streams Containing Listed Taxa, by Season and Sample Type}

\begin{tabular}{|c|c|c|c|c|c|c|c|c|c|c|c|c|c|c|c|c|}
\hline \multirow[b]{2}{*}{ Taxa } & \multicolumn{4}{|c|}{ Benthic } & \multicolumn{4}{|c|}{ Dritt } & \multicolumn{4}{|c|}{ Sticky } & \multicolumn{4}{|c|}{ Water } \\
\hline & Winter & Spring & Summer & Fall & Winter & Spring & Summer & Fall & Winter & Spring & Summer & Fall & Winter & Spring & Summer & $\mathrm{Fat}$ \\
\hline & & & & & & & \multicolumn{2}{|c|}{ Percent } & - & & & & & & & \\
\hline \multicolumn{17}{|l|}{$\begin{array}{l}\text { Platyhelminthes } \\
\text { Turbellaria } \\
\text { Tricladida }\end{array}$} \\
\hline $\begin{array}{c}\text { Planariidae } \\
\text { Nematomorpha }\end{array}$ & 25.0 & 51.7 & 45.7 & 39.6 & & 13.3 & $\begin{array}{r}17.9 \\
3.6\end{array}$ & 4.8 & & & & & & & 6.3 & \\
\hline $\begin{array}{l}\text { Nematoda } \\
\text { Annelida }\end{array}$ & 6.3 & 3.4 & 2.9 & 31.3 & & & 3.6 & & & & & & & & 125 & \\
\hline Oligochaeta & 50.0 & 75.9 & 37.1 & 54.2 & 58.3 & 33.3 & 17.9 & 4.8 & & & & & & 6.7 & & \\
\hline $\begin{array}{l}\text { Mollusca } \\
\text { Gastropoda }\end{array}$ & & 6.9 & & & 8.3 & & & & & & & & & & & \\
\hline \multicolumn{17}{|l|}{$\begin{array}{l}\text { Gastropoda } \\
\text { Anthropoda }\end{array}$} \\
\hline Amphipoda & & & & & & & & 4.8 & & & & & & & & \\
\hline Ostracoda & 6.3 & & & 10.4 & & & & & & & & & & & & \\
\hline $\begin{array}{c}\text { Arachnida } \\
\text { Araneae }\end{array}$ & & & & 42 & 8.3 & 6.7 & 17.9 & & & & 50.0 & 9.1 & & 20.0 & 100.0 & 727 \\
\hline Acarina & & & 17.1 & & 8.3 & 33.3 & 25.0 & 14.3 & & & t. & 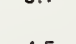 & & & 50.0 & 182 \\
\hline Opiliones & & & & & & & & & & & 8.3 & 4.5 & & & 6.3 & \\
\hline insecta & & & & & & & 3.6 & 4.8 & & & & & & & & \\
\hline $\begin{array}{l}\text { Thysanura } \\
\text { Machilidae }\end{array}$ & & & & & & & & & & & & & & & & 9.1 \\
\hline $\begin{array}{l}\text { Collembola } \\
\text { Sminthuridae }\end{array}$ & 6.3 & & & 12.5 & 8.3 & 20.0 & & 23.8 & 87.5 & 87.5 & 8.3 & 72.7 & & $\begin{array}{l}53.3 \\
13.3\end{array}$ & 87.5 & $\begin{array}{r}63.6 \\
9.1\end{array}$ \\
\hline Poduridae & & & & & & & 3.6 & & & & & & & & & \\
\hline Isotomidae & 12.5 & & & & 33.3 & 26.7 & 7.1 & 28.6 & & & & & & 33.3 & 6.3 & 36.4 \\
\hline Ephemeroptera & $\begin{array}{l}18.8 \\
375\end{array}$ & $\begin{array}{l}24.1 \\
48.3\end{array}$ & $\begin{array}{l}25.7 \\
68.6\end{array}$ & $\begin{array}{r}4.2 \\
87.5\end{array}$ & $\begin{array}{l}25.0 \\
75.0\end{array}$ & $\begin{array}{l}40.0 \\
86.7\end{array}$ & $\begin{array}{l}67.9 \\
60.7\end{array}$ & $\begin{array}{l}38.1 \\
42.9\end{array}$ & & 12.5 & $\begin{array}{r}100.0 \\
4.2\end{array}$ & 40.9 & & 6.7 & $\begin{array}{l}18.8 \\
31.3\end{array}$ & $\begin{array}{r}9.1 \\
36.4\end{array}$ \\
\hline $\begin{array}{l}\text { Siphlionuridae } \\
\text { Baetidae }\end{array}$ & $\begin{array}{l}37.5 \\
87.5\end{array}$ & $\begin{array}{l}48.3 \\
96.6\end{array}$ & $\begin{array}{l}68.0 \\
97.1\end{array}$ & $\begin{array}{l}8.5 \\
79.2\end{array}$ & 100.0 & $\begin{array}{r}80.7 \\
100.0\end{array}$ & 96.4 & 85.7 & & 6.3 & & & & 20.0 & 81.3 & 36.4 \\
\hline Heptageniidae & 100.0 & 100.0 & 100.0 & 87.5 & 100.0 & 100.0 & 78.6 & 71.4 & & 6.3 & 8.3 & & & 33.3 & 75.0 & 182 \\
\hline Leptophlebiidae & 56.3 & 82.8 & 714 & 87.5 & 66.7 & 80.0 & 57.1 & 33.3 & & & & & & & 6.3 & \\
\hline Ephemerellidae & 100.0 & 96.6 & 97.1 & 87.5 & 100.0 & 100.0 & 85.7 & 61.9 & & & 8.3 & & & 6.7 & 56.3 & \\
\hline $\begin{array}{l}\text { Odonata } \\
\text { Anisoptera }\end{array}$ & & & & & & & & & & & 4.2 & & & & & \\
\hline $\begin{array}{l}\text { Orthoptera } \\
\text { Gryllacrididae } \\
\text { Acrididae }\end{array}$ & & & & & & & & & & & 37.5 & 4.5 & & 6.7 & $\begin{array}{r}25.0 \\
6.3\end{array}$ & $\begin{array}{r}18.2 \\
9.1\end{array}$ \\
\hline $\begin{array}{l}\text { Acrididae } \\
\text { Plecoptera }\end{array}$ & 375 & 44.8 & 28.6 & 31.3 & 50.0 & 20.0 & 21.4 & 28.6 & & 6.3 & 16.7 & 13.6 & & & 6.3 & 9.1 \\
\hline Pteronarcidae & & 6.9 & 11.4 & 2.1 & & & & 4.8 & & & & & & & & \\
\hline Peltoperlidae & 75.0 & 82.8 & 77.1 & 64.6 & 75.0 & 80.0 & 39.3 & 57.1 & & & 4.2 & & & & 25.0 & 9.1 \\
\hline Taeniopterygidae & 52.5 & 72.4 & 5.7 & 6.3 & 100.0 & 80.0 & & 19.0 & & & & & & & & \\
\hline Nemouridae & 43.8 & 79.3 & 51.4 & 85.4 & 100.0 & 86.7 & 50.0 & 85.7 & 25.0 & 75.0 & 50.0 & 13.6 & & 33.3 & 81.3 & $\begin{array}{r}45.5 \\
0.1\end{array}$ \\
\hline Leuctridae & 25.0 & 37.9 & 34.3 & 27.1 & 83.3 & 86.7 & 10.7 & 14.3 & & & & & & 46.7 & & 9.1 \\
\hline Capniidae & 87.5 & 34.5 & 14.3 & 25.0 & 91.7 & 46.7 & $10: 7$ & 38.1 & & & & & & $\begin{array}{r}33.3 \\
6.7\end{array}$ & & $\begin{array}{l}9.1 \\
9.1\end{array}$ \\
\hline Pertidae & 81.3 & 79.3 & 88.6 & 91.7 & 25.0 & 13.3 & 32.1 & $\begin{array}{l}14.3 \\
47.6\end{array}$ & & & & & & $\begin{array}{l}6.7 \\
6.7\end{array}$ & $\begin{array}{c}18.8 \\
6.3\end{array}$ & 9.1 \\
\hline $\begin{array}{l}\text { Perlodidae } \\
\text { Chloroperlidae }\end{array}$ & $\begin{array}{l}37.5 \\
81.3\end{array}$ & $\begin{array}{l}48.3 \\
96.6\end{array}$ & $\begin{array}{l}80.0 \\
82.9\end{array}$ & $\begin{array}{l}58.3 \\
95.8\end{array}$ & $\begin{array}{l}33.3 \\
83.3\end{array}$ & $\begin{array}{l}26.7 \\
73.3\end{array}$ & $\begin{array}{l}42.9 \\
28.6\end{array}$ & $\begin{array}{l}47.6 \\
42.9\end{array}$ & & & 54.2 & & & 6.7 & 87.5 & 9.1 \\
\hline Psocoptera & & & & 2.1 & & & 3.6 & 23.8 & & & & & & & 81.3 & 54.5 \\
\hline Mallophaga & & & & & & & & & & & & & & & 31.3 & 9.1 \\
\hline Thysanoptera & & & 2.9 & & & & & & & & 4.2 & & & & 81.3 & \\
\hline Hemiptera & & & & & 8.3 & & 7.1 & & & & & & & & 31.3 & 9.1 \\
\hline $\begin{array}{l}\text { Gerridae } \\
\text { Coreadae }\end{array}$ & & & 2.9 & & & & $\begin{array}{l}3.6 \\
36\end{array}$ & 4.8 & & & 16.7 & & & 6.7 & 12.5 & 9.1 \\
\hline $\begin{array}{l}\text { Coreıdae } \\
\text { Saldidae }\end{array}$ & & & & & & & & 4.8 & & & 58.3 & & & & 68.8 & 9.1 \\
\hline Miridae & & & & & & & & & & & & & & & 18.8 & 9.1 \\
\hline Nabidae & & & & & & & 36 & & & & & & & & $\begin{array}{l}6.3 \\
6.3\end{array}$ & \\
\hline
\end{tabular}




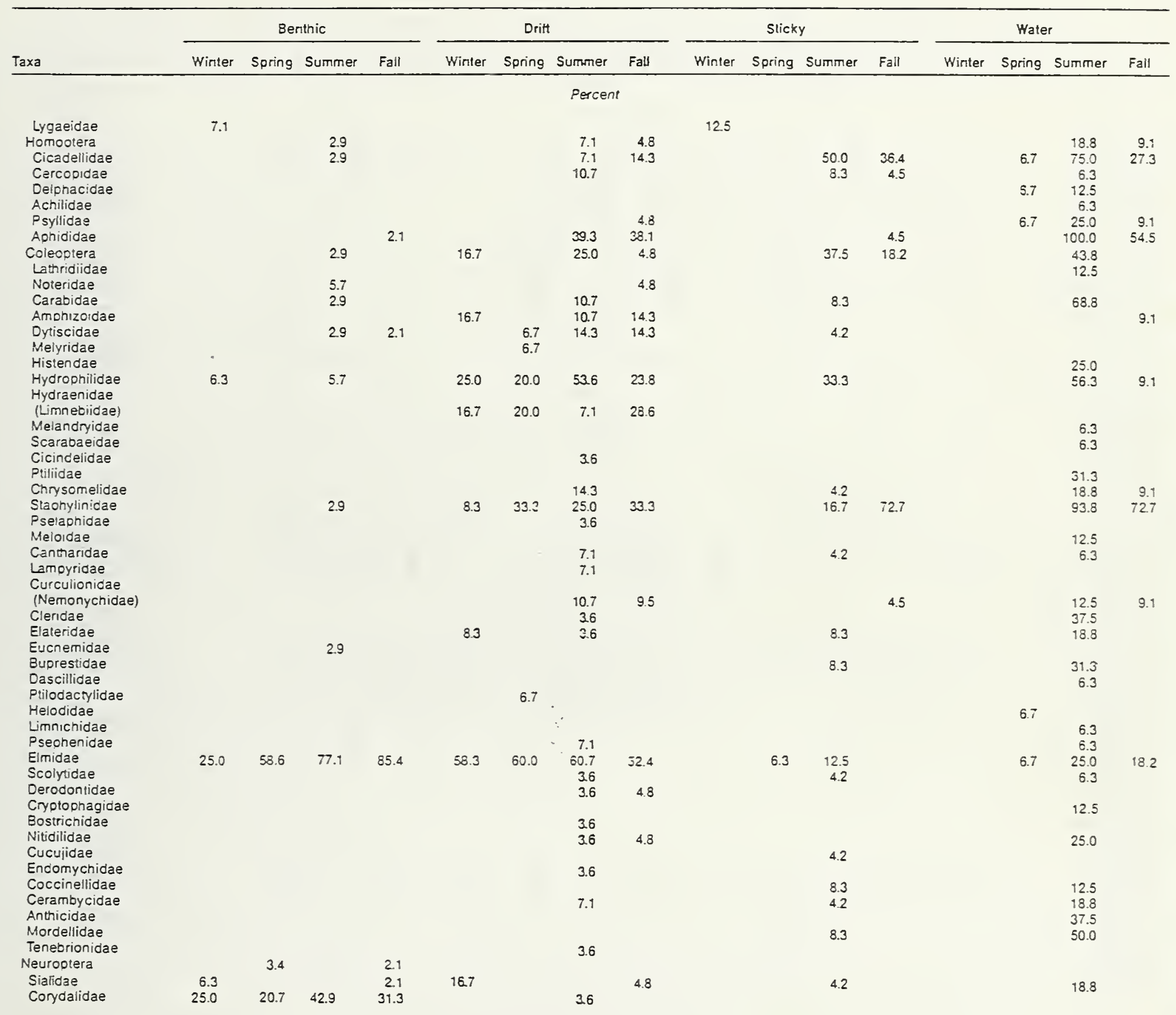

Table continued on next page.

23 


\begin{tabular}{|c|c|c|c|c|c|c|c|c|c|c|c|c|c|c|c|c|}
\hline \multirow[b]{2}{*}{ Taxa } & \multicolumn{4}{|c|}{ Benthic } & \multicolumn{4}{|c|}{ Drift } & \multicolumn{4}{|c|}{ Sticky } & \multicolumn{4}{|c|}{ Water } \\
\hline & Winter & Spring & Summer & Fall & Winter & Spring & Summer & Fall & Writer & Spring & Summer & Fall & Winter & Spring & Summer & Fall \\
\hline \multicolumn{17}{|c|}{ Percent } \\
\hline $\begin{array}{l}\text { Hemerobiidae } \\
\text { Chrysopidae }\end{array}$ & & & & & & $\begin{array}{l}6.7 \\
6.7\end{array}$ & & & & & 4.2 & & & & & \\
\hline Trichootera & 6.3 & 10.3 & 22.9 & 25.0 & 33.3 & 20.0 & 35.7 & 19.0 & 12.5 & 25.0 & 100.0 & 54.5 & & 6.7 & 18.8 & 9.1 \\
\hline Limnephilicae & 25.0 & 44.8 & 22.9 & 66.7 & 91.7 & 73.3 & 25.0 & 52.4 & & 6.3 & 12.5 & 4.5 & & & 62.5 & 54.5 \\
\hline Philopotamidae & 6.3 & & 17.1 & 6.3 & 16.7 & 26.7 & 14.3 & 9.5 & & & & & & & 87.5 & 9.1 \\
\hline Rhyacophilidae & 100.0 & 69.0 & 80.0 & 79.2 & 91.7 & 86.7 & 75.0 & 38.1 & & & & & & & 93.8 & 636 \\
\hline Hydropsychidae & 43.8 & 44.8 & 51.4 & 31.3 & 66.7 & 40.0 & 32.1 & 33.3 & & & & & & & 87.5 & \\
\hline Psycnomyidae & 6.3 & & & & & 13.3 & 7.1 & & & & & & & & & \\
\hline Polycentropodidae & 6.3 & 10.3 & 2.9 & 4.2 & 8.3 & 6.7 & & 9.5 & & & & & & & 6.3 & \\
\hline Bracnycentridae & 6.3 & 3.4 & 2.9 & 18.8 & 66.7 & 66.7 & 25.0 & 38.1 & & & & & & & & \\
\hline $\begin{array}{l}\text { Calamoceratidae } \\
\text { Lepidostomatidae }\end{array}$ & 25.0 & 27.6 & 40.0 & $\begin{array}{r}2.1 \\
29.2\end{array}$ & 750 & $\begin{array}{r}6.7 \\
60.0\end{array}$ & 32.1 & 48 & & & & & & & 563 & 91 \\
\hline $\begin{array}{l}\text { Lepidostomanidae } \\
\text { Glossosomatidae }\end{array}$ & $\begin{array}{l}25.0 \\
93.8\end{array}$ & $\begin{array}{l}27.6 \\
75.9\end{array}$ & $\begin{array}{r}40.0 \\
2.9\end{array}$ & $\begin{array}{l}29.2 \\
33.3\end{array}$ & $\begin{array}{l}75.0 \\
53.3\end{array}$ & $\begin{array}{l}50.0 \\
46.7\end{array}$ & $\begin{array}{l}32.1 \\
25.0\end{array}$ & $\begin{array}{r}4.8 \\
23.8\end{array}$ & & & & & & & $\begin{array}{l}56.3 \\
68.8\end{array}$ & 9.1 \\
\hline $\begin{array}{l}\text { Pnrygane:dae } \\
\text { Hydroptilidae }\end{array}$ & & & 2.9 & & & & & & & & & & & & 313 & \\
\hline Leoidoptera & & & & 0.3 & & 6.7 & $\begin{array}{r}3.6 \\
10.7\end{array}$ & $\begin{array}{l}4.8 \\
4.8\end{array}$ & & & 16.7 & 182 & & & $\begin{array}{l}31.3 \\
25.0\end{array}$ & \\
\hline Pieridae & & & & & & 0.8 & & & & & 10.1 & 10.2 & & & 6.3 & \\
\hline Lycaenidae & & & & & & & & & & & & & & & 12.5 & \\
\hline Hesperidae & & & & & & & & & & & & & & & 6.3 & \\
\hline Geometridae & & & & & & & 3.6 & & & & & & & & 18.8 & 9.1 \\
\hline Microlepidoptera & & & & & & & 14.3 & & & & 8.3 & & & & 43.8 & \\
\hline Pyralidae & & & & & & & 7.1 & & & & & & & & & \\
\hline $\begin{array}{l}\text { Aegeriidae } \\
\text { (Sesiidae) }\end{array}$ & & & & & & & & & & & 125 & & & & & \\
\hline Diptera & & 3.4 & 8.6 & & & 13.3 & 17.9 & 0.5 & 125 & 12.5 & 4.2 & 9.1 & & 13.3 & $\begin{array}{l}12.5 \\
25.0\end{array}$ & 273 \\
\hline ivematocera & & & & & & & & & 50.0 & & & 45.5 & & & & 27.0 \\
\hline Tanyderidae & & & & & & & & & & & & & & & 12.5 & 18.2 \\
\hline Tipulidae & 68.8 & 89.7 & 82.9 & 91.7 & 91.7 & 66.7 & 64.3 & 42.9 & 12.5 & 25.0 & 83.3 & 54.5 & & 80.0 & 93.8 & 36.4 \\
\hline Psychodiriae & 12.5 & & & 2.1 & 25.0 & 13.3 & 7.1 & 14.3 & & 6.3 & 12.5 & 4.5 & & 20.0 & 81.3 & 36.4 \\
\hline Blephariceridae & 6.3 & 3.4 & & & 66.7 & 60.0 & 7.1 & & & & 45.8 & & & 6.7 & 43.8 & \\
\hline Deuterophlebiidae & & & & & & 13.3 & & & & & & & & & & \\
\hline Dixidae & & & 5.7 & & 8.3 & 20.0 & 25.0 & 28.6 & 12.5 & & & & & 6.7 & 62.5 & 53.6 \\
\hline Culicıdae & & & & & & & & & & & & & & & 6.3 & \\
\hline Ceratopogonidae & & 17.2 & 20.0 & 25.0 & 25.0 & & 25.0 & 14.3 & & & 4.2 & 9.1 & & & 100.0 & \\
\hline Chironomidae & 68.8 & 86.2 & 100.0 & 97.9 & 91.7 & 100.0 & 89.3 & 90.5 & 500 & 68.8 & 100.0 & 68.2 & & 100.0 & 100.0 & 90.9 \\
\hline Simuliidae & 18.6 & 37.9 & 20.0 & 2.1 & 100.0 & 93.3 & 57.1 & 19.0 & 125 & 12.5 & 125 & & & i3.3 & 31.3 & \\
\hline Bibionidae & & & & & & 6.7 & & & & & & & & & & \\
\hline Mycetopnilidae & & & & & 8.3 & 46.7 & 25.0 & 28.6 & 37.5 & 43.8 & 83.3 & 95.5 & & 60.0 & 87.5 & 90.9 \\
\hline Sciaridae & & & 5.7 & & & 26.7 & 57.1 & 28.6 & & 18.8 & 79.2 & 54.5 & & 13.3 & 100.0 & 636 \\
\hline Cedidiomyiidae & & & & & & & & & & & 42 & 4.5 & & & 100.0 & 54.5 \\
\hline \multicolumn{17}{|l|}{ Srachycera } \\
\hline Stratiomyidae & & & & & & & & 4.8 & & & & & & & & \\
\hline Tabanidae & & & 2.9 & & & & & & & & & & & & 6.3 & \\
\hline Rhagıcnidae & & & & & & & 3.6 & & & & & & & & 12.5 & \\
\hline Perıscelididae & & & & & & & & & & & & & & & 6.3 & \\
\hline Asilidae & & & & & & & & & & & 50.0 & & & & 50.0 & \\
\hline Acroceridae & & & & & & & & & & & & & & & 31.3 & \\
\hline Bombyliidae & & & & & & & & & & & & & & & 12.5 & \\
\hline Empididae & 6.3 & 34.5 & 54.3 & 31.3 & 25.0 & 33.3 & 75.0 & 19.0 & 75.0 & 87.5 & 95.8 & 100.0 & & 86.7 & 100.0 & 81.8 \\
\hline Dolichopodioae & & & 2.9 & & & 13.3 & 7.1 & & 12.5 & 6.3 & 100.0 & 36.4 & & 6.7 & 75.0 & 27.3 \\
\hline $\begin{array}{l}\text { Cyclormapha } \\
\text { Lonchopieridae }\end{array}$ & & & & & & & & & & & & & & & 43.8 & \\
\hline Phoridae & & & & & 8.3 & 6.7 & 21.4 & 14.3 & & & 8.3 & 4.5 & & 33.3 & 87.5 & 54.5 \\
\hline Platypezidae & & & & & & & & & 500 & & & 45.5 & & & & \\
\hline Pipunculidae & & & & & & & & & & & 4.2 & & & & & \\
\hline
\end{tabular}

Table continued on next page. 


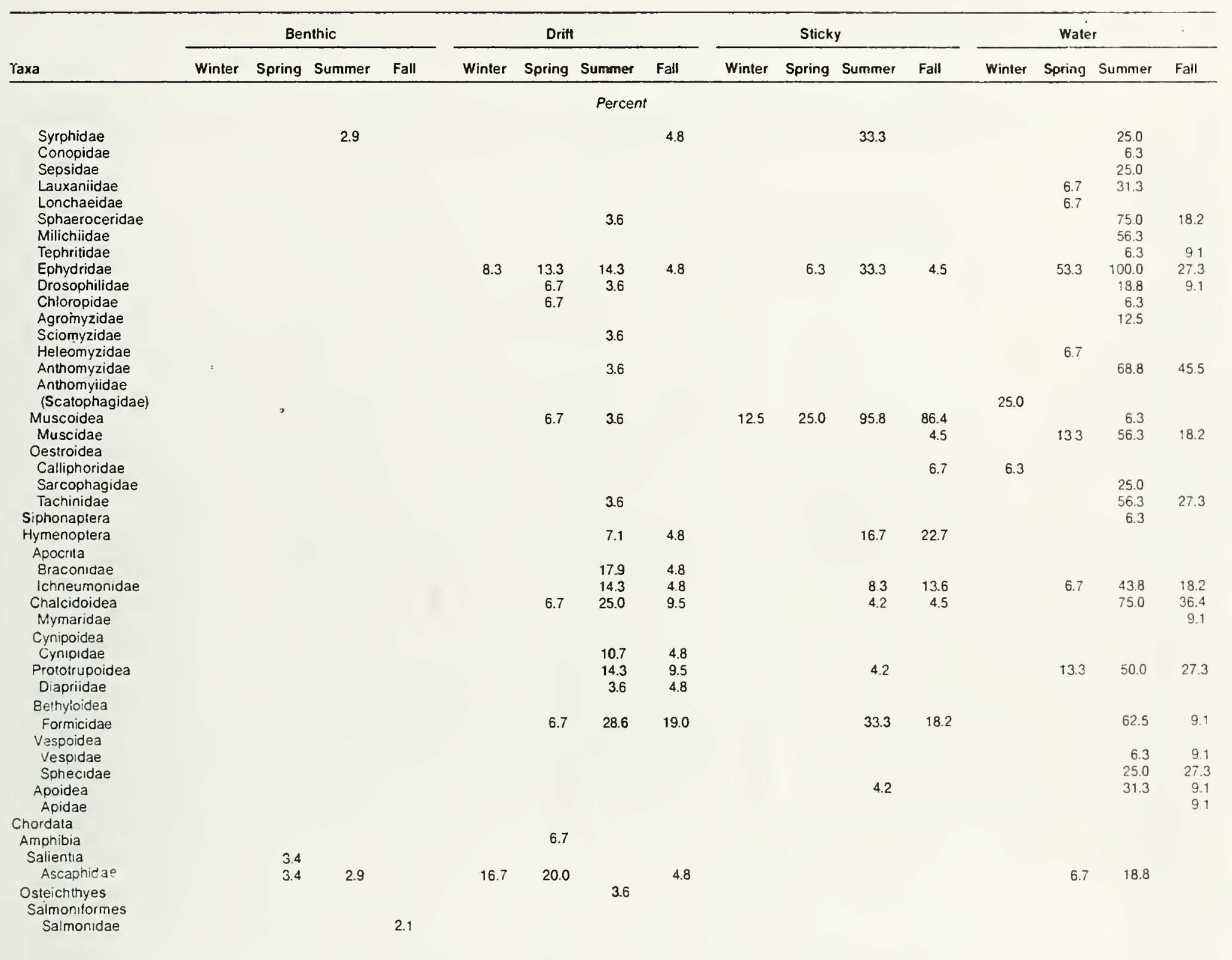

Table continued on next page. 
Percentage of Samples From Central Streams Containing Listed Taxa, by Season and Sample Type

\begin{tabular}{|c|c|c|c|c|c|c|c|c|c|c|c|c|c|c|c|c|}
\hline \multirow[b]{2}{*}{ Taxa } & \multicolumn{4}{|c|}{ Benthic } & \multicolumn{4}{|c|}{ Dritt } & \multicolumn{4}{|c|}{ Sticky } & \multicolumn{4}{|c|}{ Water } \\
\hline & Winter & Spring & Summer & Fall & Winter & Spring & Summer & Fall & Winter & Spring & Summer & Fall & Winter & Spring & Summer & Fal \\
\hline & & & & & & & Percent & & - & & & & & & & \\
\hline \multirow{2}{*}{\multicolumn{17}{|c|}{$\begin{array}{l}\text { Platyhelminthes } \\
\text { Turbellaria }\end{array}$}} \\
\hline & & & & & & & & & & & & & & & & \\
\hline $\begin{array}{l}\text { Tricladida } \\
\text { Planariidae }\end{array}$ & \multicolumn{16}{|c|}{ Tricladida } \\
\hline Nematoda & & 6.7 & 10.4 & 11.1 & 12.5 & 26.7 & 4.3 & 12.0 & & & & & & & 91 & \\
\hline Annelida & & & & & & & & & & & & & & & & \\
\hline $\begin{array}{l}\text { Oligochaeta } \\
\text { Mollusca }\end{array}$ & 56.3 & 60.0 & 50.0 & 75.6 & 12.5 & 26.7 & 4.3 & 20.0 & & & & & & 9.1 & & \\
\hline Gastropoda & & & & & & & & & & & & & & & 9.1 & \\
\hline Basommatophora & & & & & & & & & & & & & & & 3.1 & \\
\hline Physidae & & & & 6.7 & & & & & & & & & & & & \\
\hline Planorbidae & & & & 2.2 & & & 4.3 & & & & & & & & & \\
\hline Mesogastropoda & & & & & & & & & & & & & & & & \\
\hline Hydrobiidae & & & 4.2 & 2.2 & & 6.7 & 4.3 & & & & & & & & & \\
\hline Pelecypoda & & & & & & 6.7 & & & & & & & & & & \\
\hline Sphaeridae & 6.3 & & & 4.4 & & & & & & & & & & & & \\
\hline Arthropoda & & & & & & & & & & & & & & & & \\
\hline Crustacea & & & & & & & & & & & & & & & & \\
\hline Amphipoda & & 3.3 & 4.2 & 2.2 & & & & & & & & & & & & \\
\hline Ostracoda & & & & 2.2 & & & & & & & & & & & & \\
\hline Copepoda & & & & 2.2 & & & & & & & & & & & & \\
\hline Arachnida & & & & & & & & & & & 72.7 & 41.7 & & & $i 2.7$ & 73.3 \\
\hline Araneae & & & 2.1 & $\begin{array}{l}4.4 \\
6.7\end{array}$ & & 13.3 & $\begin{array}{l}39.1 \\
130\end{array}$ & $\begin{array}{l}360 \\
280\end{array}$ & & $\begin{array}{r}28.6 \\
7.1\end{array}$ & 2.1 & 12.5 & & $\begin{array}{r}66.4 \\
9.1\end{array}$ & 54.5 & 6.7 \\
\hline $\begin{array}{l}\text { Acarına } \\
\text { Pseudoscorpıonida }\end{array}$ & 6.3 & & 14.6 & 6.7 & & & 13.0 & 200 & & 1.1 & 4.5 & 8.0 & & & 9.1 & \\
\hline Opiliones & & & & & & & & & & & & 4.2 & & & & \\
\hline Insecta & & & & & & 6.7 & & & & 14.3 & 4.5 & & & & & \\
\hline $\begin{array}{l}\text { Machilicae } \\
\text { Collembola }\end{array}$ & & & & 2.2 & & & 4.3 & 240 & & 57.1 & 4.5 & 12.5 & & & 9.1 & 6.7 \\
\hline Sminthuridae & & & & & & 6.7 & & & & 21.4 & & 8.3 & & 36.4 & & \\
\hline Isotomidae & & & & & & 13.3 & 8.7 & 8.0 & & 42.9 & 18.2 & 20.8 & & 81.8 & 54.5 & 86.7 \\
\hline Ephemeroptera & & & 10.4 & 8.9 & & & 39.1 & 20.0 & & 21.4 & 86.4 & 41.7 & & & 27.3 & 40.0 \\
\hline Siphlonuridae & 18.8 & 3.3 & 52.1 & 244 & 62.5 & 60.0 & 47.8 & 40.0 & & 7.1 & 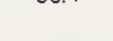 & 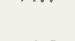 & & & & 26.7 \\
\hline Baetidae & 75.0 & 86.7 & 93.8 & 77.8 & 75.0 & 100.0 & 78.3 & 880 & & 7.1 & & 4.2 & & & 9.1 & 60.0 \\
\hline Heplageniidae & 87.5 & 80.0 & 83.3 & 91.1 & 75.0 & 93.3 & 56.5 & 880 & & 14.3 & & & & 27.3 & 27.3 & 13.3 \\
\hline Leptophlebiidae & 56.3 & 36.7 & 20.8 & 46.7 & 50.0 & 13.3 & 43.5 & 520 & & & & & & & 9.1 & 6.7 \\
\hline Ephemerellidae & 87.5 & 83.3 & 91.7 & 91.1 & 75.0 & 86.7 & 826 & 84.0 & & 7.1 & 13.6 & & & 9.1 & 27.3 & 13.3 \\
\hline Odonata & 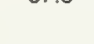 & & 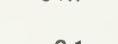 & & & & & & & & & & & & & \\
\hline Gomphidae & 63 & & 2.1 & 44 & & & & & & & & & & & & \\
\hline $\begin{array}{l}\text { Cordulegastridae } \\
\text { Libellulidae }\end{array}$ & 6.3 & & & 4.4 & & 6.7 & & & & & & & & & & \\
\hline Orthoptera & 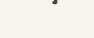 & & 2.1 & & & & 8.7 & & & & 18.2 & & & & & 67 \\
\hline Tetrigıdae & & & & & & & & 4.0 & & & & & & 9.1 & & 2.7 \\
\hline $\begin{array}{l}\text { Gryllidae } \\
\text { Dermaptera }\end{array}$ & & & & & & & 4.3 & & & & & & & & & \\
\hline Forticulidae & & & & & & & & & & & & 4.2 & & & & 6.7 \\
\hline Plecoptera & & & 16.7 & 2.2 & & & 8.7 & 80 & & 42.9 & 18.2 & 12.5 & & & & 6.7 \\
\hline Peltoperlidae & 6.3 & 3.3 & 2.1 & 6.7 & & & & 8.0 & & & & & & & & \\
\hline Taeniopterygidae & & & & 2.2 & & & & & & & & & & & 9.1 & \\
\hline Nemouridae & 100.0 & 76.7 & 31.3 & 66.7 & 87.5 & 93.3 & 21.7 & 92.0 & & 71.4 & 31.8 & 12.5 & & 90.9 & 36.4 & 40.0 \\
\hline Leuctridae & 6.3 & 10.0 & & 11.1 & & & & 8.0 & & 8.1 & & & & 27.3 & & \\
\hline Capniidae & 50.0 & 16.7 & & 53.3 & 62.5 & 20.0 & 8.7 & 72.0 & & 7.1 & & & & 36.4 & & 13.3 \\
\hline Perlidae & 75.0 & 73.3 & 81.3 & 822 & & & 13.0 & 24.0 & & & & & & & & \\
\hline Perlodidae & 31.3 & 6.7 & 41.7 & 71.1 & & 13.3 & 30.4 & 20.0 & & & & & & 27.3 & 18.2 & \\
\hline Chloroperlidae & 81.3 & 76.7 & 89.6 & 91.1 & 62.5 & & 21.7 & 60.0 & & & 22.7 & & & & 9.1 & \\
\hline Psocoptera & & & 2.1 & 2.2 & & & 8.7 & 12.0 & & & & & & & 9.1 & 6.7 \\
\hline Thysanoptera & & & & & & 6.7 & & 4.0 & & & 4.5 & & & & 63.6 & 267 \\
\hline Hemiptera & & . & 10.4 & & & & 13.0 & 120 & & 7.1 & 18.2 & 4.2 & & & 27.3 & 26.7 \\
\hline Cornxidae & & & & & 12.5 & & 13.0 & 80 & & & & & & & & \\
\hline Nerdidae & & & & & & & $\begin{array}{r}4.3 \\
26.1\end{array}$ & & & 7.1 & 13.6 & 4.2 & & 9.1 & 9.1 & 6.7 \\
\hline $\begin{array}{l}\text { Gerrndae } \\
\text { Veliidae }\end{array}$ & & & 2.1 & & & & $\begin{array}{r}26.1 \\
4.3\end{array}$ & $\begin{array}{r}12.0 \\
8.0\end{array}$ & & 7.1 & 13.6 & 4.2 & & 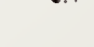 & . & \\
\hline Saldidae & & & & & & & 4.3 & 0.0 & & & 63.6 & & & & 54.5 & 6.7 \\
\hline Anthocoridae & & & & & & & 8.7 & & & & & & & & & \\
\hline Miridae & & & & & & & 8.7 & 4.0 & & & & & & & 27.3 & \\
\hline Nabidae & & & & & & & 8.7 & & & & & & & & 9.1 & \\
\hline Reduviidae & & & & & & & & 4.0 & & & 4.5 & & & & & \\
\hline Tingldae & & & & & & & 8.7 & & & & & & & & & \\
\hline Aradidae & & & & & & & $\begin{array}{l}4.3 \\
87\end{array}$ & & & & & & & & 27.3 & \\
\hline Lygaeidae & & & & & & & 8.7 & & & & & & & & $2 \pi, 0$ & \\
\hline
\end{tabular}

Table continued on next page. 


\begin{tabular}{|c|c|c|c|c|c|c|c|c|c|c|c|c|c|c|c|c|}
\hline \multirow[b]{2}{*}{ Taxa } & \multicolumn{4}{|c|}{ Benthic } & \multicolumn{4}{|c|}{ Drift } & \multicolumn{4}{|c|}{ Sticky } & \multicolumn{4}{|c|}{ Water } \\
\hline & Winter & Spring & Summer & Fall & Winter & Spring & Summer & Fall & Winter & Spring & Summer & Fall & Winter & Spring & Summer & Fall \\
\hline \multicolumn{17}{|c|}{ Percent } \\
\hline Homoptera & & & 2.1 & 2.2 & & & 8.7 & 8.0 & & 14.3 & 27.3 & 12.5 & & 36.4 & 36.4 & 6.7 \\
\hline Cicadellidae & & 3.3 & 27.1 & 13.3 & & & 52.2 & 80.0 & & 28.6 & 81.8 & 91.7 & & 45.5 & 63.6 & 73.3 \\
\hline Cercopidae & & & 6.3 & & & & 4.3 & 4.0 & & & & 4.2 & & 9.1 & & \\
\hline Delphacidae & & & & & & & & & & & & & & & 9.1 & \\
\hline Psyllidae & & 3.3 & & 2.2 & & & 26.1 & 28.0 & & 7.1 & 9.1 & 29.2 & & & 45.5 & 53.3 \\
\hline Aphididae & & & 10.4 & 2.2 & & 6.7 & 52.2 & 68.0 & & 28.6 & 31.8 & 45.8 & & & 72.7 & 93.3 \\
\hline Eriosomatidae & & & & & & & 4.3 & & & & & & & & & 33.3 \\
\hline Coccoidea & & & & & & & & 8.0 & & & & & & 9.1 & & \\
\hline Coleoptera & & & & & & & 30.4 & 12.0 & & 7.1 & 36.4 & 16.7 & & 18.2 & 27.3 & 13.3 \\
\hline Cupedidae & & & & & & & & & & & & & & & 18.2 & \\
\hline Silphidae & & & & & & & 4.3 & & & & & & & & & \\
\hline Lathridiidae & & & & & & & 4.3 & & & & & & & & & \\
\hline Carabidae & & & & & & & 4.3 & 12.0 & & & 4.5 & 4.2 & & 9.1 & & \\
\hline Haliplidae & & & & 2.2 & & & 4.3 & & & & & & & & & \\
\hline Amphizoidae & & & & & & & 4.3 & 16.0 & & & & & & & & \\
\hline $\begin{array}{l}\text { Dytıscidae } \\
\text { Gyrınıdae }\end{array}$ & 12.5 & 6.7 & 6.3 & 20.0 & & 6.7 & 52.2 & $\begin{array}{r}32.0 \\
4.0\end{array}$ & & & 9.1 & & & & & \\
\hline Hydrophilidae & 6.3 & & & & 12.5 & 6.7 & 60.9 & 40.0 & & & 4.5 & 4.2 & & 18.2 & 18.2 & 6.7 \\
\hline $\begin{array}{l}\text { Hydraenidae } \\
\text { (Limnebiidae) }\end{array}$ & & 6.7 & 2.1 & & 12.5 & & 21.7 & 28.0 & & & & & & 36.4 & 9.1 & \\
\hline Melandryıdae & & 0.1 & 2.1 & & 12.0 & & 4.3 & 20.0 & & & & & & & 9.1 & \\
\hline Scarabaeıdae & & & & & & & 13.0 & & & & 4.5 & & & & 9.1 & \\
\hline Ptiliidae & & & & & & & & & & & 4.5 & & & & 27.3 & 20.0 \\
\hline Chrysomelidae & & 3.3 & 2.1 & & & & 43.5 & 12.0 & & 21.4 & 22.7 & 20.8 & & 54.5 & 18.2 & 33.3 \\
\hline Scaphididae & & & & & & & 8.7 & & & & & & & & & 13.3 \\
\hline Staphylinidae & & & & & & 13.3 & 39.1 & 44.0 & & 14.3 & 27.3 & 58.3 & & 72.7 & 45.5 & 46.7 \\
\hline Colydiidae & & & & & & & & & & & & & & & 9.1 & \\
\hline $\begin{array}{l}\text { Scydmaenidae } \\
\text { Cantharidae }\end{array}$ & & & & & & & & 4.0 & & & & & & & 182 & \\
\hline & & & & & & & 8.7 & & & & & & & & 18.2 & \\
\hline (Nemonychidae) & & & & & & & 8.7 & & & & 4.5 & 4.2 & & 9.1 & 182 & \\
\hline Malachiidae & & & & & & & & & & & & & & & 91 & \\
\hline Clerıdae & & & & & & & 4.3 & 8.0 & & & & & & 9.1 & 9.1 & \\
\hline Elateridae & & & & & & & & & & & & & & & 9.1 & \\
\hline Buprestidae & & & & & & & & & & 17.4 & 4.0 & & & 9.1 & & \\
\hline Helodidae & & & & & & & & 4.0 & & & & & & & & \\
\hline Dryopidae & & & & & & & 4.3 & 12.0 & & & & & & & & \\
\hline Elmidae & 87.5 & 70.0 & 91.7 & 93.3 & 37.5 & 26.7 & 65.2 & 84.2 & & & 18.2 & 4.2 & & & 27.3 & \\
\hline Scolytidae & & & & & & & 4.3 & 4.0 & & & & & & & 9.1 & 6.7 \\
\hline Derodontidae & & & & & & & & & & & 4.5 & & & & & 6.7 \\
\hline Nitidulidae & & & & & & & & & & & & & & 9.1 & 27.3 & 6.7 \\
\hline Coccinellidae & & & & & & & 4.3 & & & & & & & & & \\
\hline Cerambycidae & & & 2.1 & & & & 13.0 & & & & & & & & 18.2 & \\
\hline Pedilidae & & & & & & & & 4.0 & & & & & & & & \\
\hline Cephaloidae & & & & & & & & & & & & & & & 9.1 & \\
\hline Mordellidae & & & & & & & 4.3 & & & & 9.1 & & & & 9.1 & \\
\hline Tenebrionidae & & & & & & & 8.7 & & & & & & & & & \\
\hline Neuroptera & & & & & & & & & & & & 4.2 & & & & \\
\hline Sialidae & & 3.3 & & & & & & & & & & & & & & \\
\hline Raphidiidae & & & & & & & 4.3 & & & & 4.5 & & & & & \\
\hline Conıopterygidae & & & & & & & 4.3 & & & & & & & & & \\
\hline Hemerobiidae & & & & & & & & 4.0 & & & & & & & & \\
\hline Trichoptera & 25.0 & 6.7 & 37.5 & 24.4 & 12.5 & & 30.4 & 28.0 & & 21.4 & 80.9 & 66.7 & & & 27.3 & 6.7 \\
\hline Limnephilidae & 62.5 & 76.7 & 85.4 & 55.6 & 62.5 & 80.0 & 78.3 & 76.0 & & & 13.6 & 29.2 & & 18.2 & 27.3 & 53.3 \\
\hline Philopotamidae & & & 14.6 & 2.2 & & & 4.3 & 12.0 & & & & & & & 27.3 & 60.0 \\
\hline Rhyacophilidae & 56.3 & 76.7 & 45.8 & 71.1 & 12.5 & 26.7 & 13.0 & 40.0 & & & & & & 18.2 & 72.7 & 60.0 \\
\hline Hydropsychidae & 68.8 & 66.7 & 50.0 & 73.3 & 37.5 & 13.3 & 26.1 & 52.0 & & & & & & & 36.4 & 26.7 \\
\hline Psychomyiidae & & & & 2.2 & & & 4.3 & & & & & & & & & 6.7 \\
\hline
\end{tabular}

Table continued on next page. 


\begin{tabular}{|c|c|c|c|c|c|c|c|c|c|c|c|c|c|c|c|c|}
\hline \multirow[b]{2}{*}{ Taxa } & \multicolumn{4}{|c|}{ Benthic } & \multicolumn{4}{|c|}{ Dritt } & \multicolumn{4}{|c|}{ Sticky } & \multicolumn{4}{|c|}{ Water } \\
\hline & Winter & Spring & Summer & Fall & Winter & Spring & Summer & Fall & Winter & Spring & Summer & Fall & Winter & Spring & Summer & Fall \\
\hline \multicolumn{17}{|c|}{ Percent } \\
\hline Polycentropodidae & & & & 4.4 & & 6.7 & & & & & & & & & & \\
\hline Brachycentridae & 43.8 & 43.3 & 14.6 & 42.2 & 25.0 & 33.3 & 26.1 & 44.0 & & & & & & & 18.2 & 6.7 \\
\hline Lepidostomatidae & 56.3 & 36.7 & 16.7 & 51.1 & 25.0 & 53.3 & 43.5 & 48.0 & & & & 4.2 & & 9.1 & 36.4 & 6.7 \\
\hline $\begin{array}{l}\text { Glossosomatidae } \\
\text { Phryganeidae }\end{array}$ & 25.0 & 3.3 & 47.9 & 26.7 & & & 17.4 & 4.0 & & & & & & & 9.1 & $\begin{array}{l}20.0 \\
13.3\end{array}$ \\
\hline Lepidoptera & & & 2.1 & & & 6.7 & 21.7 & 16.0 & & 7.1 & 31.8 & 29.2 & & & 27.3 & 6.7 \\
\hline $\begin{array}{l}\text { Saryridae } \\
\text { Hesperiidae }\end{array}$ & & & & & & & & & & & & & & & & $\begin{array}{r}6.7 \\
26.7\end{array}$ \\
\hline Arctiidae & & & & & & & & & & & & & & & 18.2 & 13.3 \\
\hline Noctuidae & & & & & & & & & & & & & & 9.1 & 45.5 & 13.3 \\
\hline Geometridae & & & & & & & & & & & & & & & 9.1 & 6.7 \\
\hline Microlepidoptera & & & & & & & 17.4 & 8.0 & & 7.1 & 18.2 & 4.2 & & & 27.3 & \\
\hline Diptera & & 3.3 & 12.5 & 4.4 & 12.5 & & 43.5 & 36.0 & & 50.0 & 36.4 & 25.0 & & & 27.3 & 20.0 \\
\hline Nematocera & & & & & & & & & & 28.6 & 45.5 & 25.0 & & & & 6.7 \\
\hline Tipulidae & 93.8 & 80.0 & 77.1 & 80.0 & 12.5 & 40.0 & 43.5 & 28.0 & & 21.4 & 86.4 & 37.5 & & 36.4 & 72.7 & 46.7 \\
\hline Psychodidae & 81.3 & 63.3 & 4.2 & 91.1 & 62.5 & 20.0 & 26.1 & 64.0 & & 7.1 & 27.3 & 4.2 & & & 63.6 & 20.0 \\
\hline Blephariceridae & & 10.0 & & & & 6.7 & & & . & & 4.5 & & & & 18.2 & 6.7 \\
\hline $\begin{array}{l}\text { Dixidae } \\
\text { Culicidae }\end{array}$ & & & & & & & 34.8 & $\begin{array}{r}28.0 \\
4.0\end{array}$ & & 14.3 & 13.6 & 16.7 & & 9.1 & 27.3 & 73.3 \\
\hline Ceratopogonidae & 25.0 & 23.3 & 22.9 & 51.1 & & & 26.1 & 20.0 & & 7.1 & 4.5 & 4.2 & & & 63.6 & 33.3 \\
\hline Chironomidae & 100.0 & 96.7 & 100.0 & 95.6 & 100.0 & 86.7 & 95.7 & 92.0 & & 100.0 & 100.0 & 100.0 & & 81.8 & 90.9 & 100.0 \\
\hline Simuliidae & 18.8 & 70.0 & 18.8 & & 37.5 & 66.7 & 65.2 & 16.0 & & 7.1 & 13.6 & 4.2 & & 27.3 & 63.6 & 13.3 \\
\hline Bibionidae & & & & & & & & 24.0 & & & & & & 72.7 & 727 & $\begin{array}{r}20.0 \\
100.0\end{array}$ \\
\hline Mycetophilidae & & & & 2.2 & & & 26.1 & 44.0 & & 35.7 & $\begin{array}{l}50.9 \\
40.9\end{array}$ & $\begin{array}{l}75.0 \\
83.3\end{array}$ & & 27.3 & $\begin{array}{l}2.1 \\
72.7\end{array}$ & 93 \\
\hline $\begin{array}{l}\text { Sciaridae } \\
\text { Cedidiomyiidae }\end{array}$ & & & & & & & $\begin{array}{l}69.6 \\
34.8\end{array}$ & $\begin{array}{r}56.0 \\
40\end{array}$ & & 14.3 & 4.5 & $\begin{array}{l}83.3 \\
12.5\end{array}$ & & 9.1 & 72.7 & 46.7 \\
\hline $\begin{array}{l}\text { Cedidiomyiidae } \\
\text { Brachycera }\end{array}$ & & & & & & & & & & & & & & & & \\
\hline $\begin{array}{l}\text { Brachycera } \\
\text { Xylomyidae }\end{array}$ & & & & & & & & & & & 4.5 & & & & & \\
\hline Stratiomyidae & 6.3 & & 2.1 & 2.2 & & & & & & & 4.5 & & & & & \\
\hline Tabanidae & 12.5 & 3.3 & 16.7 & 17.8 & & 6.7 & 8.7 & & & & 4.5 & & & & & \\
\hline Rhagionidae & & & & & & & & & & & 13.6 & & & & 27.3 & \\
\hline Therevidae & & & & & & & & & & & 9.1 & & & & & 6.7 \\
\hline Asilidae & & & & & & & & & & & 0.1 & 4.2 & & & 9.1 & 13.3 \\
\hline Acroceridae & & & & & & & & & & & & & & & 27.3 & \\
\hline Empididae & 18.8 & 16.7 & 18.8 & 46.7 & & & 43.5 & 24.0 & & 50.0 & 95.5 & 87.5 & & 18.2 & 72.7 & 86.7 \\
\hline $\begin{array}{l}\text { Dolichopodidae } \\
\text { Cyclorrhapha }\end{array}$ & & & 2.1 & 4.4 & & & 8.7 & 160 & & 14.3 & 100.0 & 66.7 & & 9.1 & $\begin{array}{r}63.6 \\
9.1\end{array}$ & 33.3 \\
\hline $\begin{array}{l}\text { Cyclorrhapha } \\
\text { Lonchopteridae }\end{array}$ & & & 2.1 & & & & & & & & & & & & & 6.7 \\
\hline $\begin{array}{l}\text { Phoridae } \\
\text { Pal }\end{array}$ & & & & & & & 13.0 & 120 & & 7.1 & 18.2 & 12.5 & & 45.5 & 36.4 & 40.0 \\
\hline Pipunculidae & & & & & & & 4.3 & & & & 4.5 & & & & & 20.0 \\
\hline Syrphidae & & & & & & & & 8.0 & & & 22.7 & & & & 9.1 & 6.7 \\
\hline Sepsidae & & & & & & & & & & 3.1 & & & & & 9.1 & 6.7 \\
\hline Lonchaeidae & & & & & & & & & & & & & & 9.1 & & \\
\hline Sphaeroceridae & & & & & & & & 4.0 & & & & & & 182 & & $\begin{array}{r}6.7 \\
73.3\end{array}$ \\
\hline Ephydridae & & & 4.2 & & & & 39.1 & & & 21.4 & 27.3 & 20.8 & & 35.4 & 72.1 & \\
\hline
\end{tabular}

Table continued on next page. 


\begin{tabular}{|c|c|c|c|c|c|c|c|c|c|c|c|c|c|c|c|c|}
\hline \multirow[b]{2}{*}{ Taxa } & \multicolumn{4}{|c|}{ Benthic } & \multicolumn{4}{|c|}{ Dritt } & \multicolumn{4}{|c|}{ Sticky } & \multicolumn{4}{|c|}{ Water } \\
\hline & Winter & Spring & Summer & Fall & Winter & Spring & Summer & Fall & Winter & Spring & Summer & Fall & Winter & Spring & Summer & Fall \\
\hline \multicolumn{17}{|c|}{ Percent } \\
\hline Drosophilidae & & & & & & & 8.7 & 120 & & & & & & & 18.2 & 26.7 \\
\hline Chloropidae & & & & & & & 13.0 & 4.0 & & & 13.6 & & & & 18.2 & 6.7 \\
\hline $\begin{array}{l}\text { Agromyzidae } \\
\text { Heleomyzidae }\end{array}$ & & & & & & & 4.3 & & & & & & & & & $\begin{array}{l}\varepsilon .7 \\
6.7\end{array}$ \\
\hline Anthomyzidae & & & & & & & 4.3 & & & & & & & & & \\
\hline Anthomyiidae & & & & & & & & & & & & & & & & \\
\hline (Scatophagidae) & & & & & & & 13.0 & 24.0 & & 14.3 & 45 & 8.3 & & 36.4 & 54.5 & 73.3 \\
\hline Muscoidea & & & & & & & & 4.0 & & 28.6 & 90.9 & 62.5 & & & 9.1 & \\
\hline Muscidae & & & & & & & & & & & 9.1 & 42 & & & 36.4 & 33.3 \\
\hline Oestroidea & & & & & & & & & & & 364 & & & & & \\
\hline Calliohoridae & & & & & & & - & & & & & 42 & & 45.5 & 18.2 & 33.3 \\
\hline Sarcophagidae & & & 4.2 & & & & & & & & & & & & 91 & \\
\hline Tachinidae & & & & & & & 43 & & & & & 42 & & 9.1 & 27.3 & 46.7 \\
\hline Hymenoptera & & & 4.2 & 22 & & & 13.0 & 40.0 & & 7.1 & 27.3 & 8.3 & & & $i 8.2$ & 6.7 \\
\hline $\begin{array}{l}\text { Symphyta } \\
\text { Apocrita }\end{array}$ & & & & & & & & & & & & & & & 9.1 & \\
\hline Braconidae & & & 2.1 & & & & 43.5 & 24.0 & & 7.1 & 4.5 & & & & 18.2 & 26.7 \\
\hline Ichneumonidae & & & 2.1 & & & & 21.7 & 120 & & 7.1 & 31.8 & 16.7 & & 182 & 45.5 & 6.7 \\
\hline Chalcidoidea & & & & & & & 34.8 & 16.0 & & 7.1 & & 8.3 & & & 27.3 & 200 \\
\hline Mymaridae & & & & 22 & & & 4.3 & & & & 4.5 & & & & 18.2 & 6.7 \\
\hline Pteromalidae & & & & & & & 4.3 & 4.0 & & & & & & & & \\
\hline Cynipoidea & & & & & & & 4.3 & & & & & & & & 18.2 & \\
\hline Cynipidae & & & & & & & & 4.0 & & & & & & & & \\
\hline Evaniidae & & & & & & & 4.3 & & & & & & & & 9.1 & \\
\hline Proclotrupoidea & & & 2.1 & & & & 13.0 & 16.0 & & & 182 & 12.5 & & 9.1 & 36.4 & 46.7 \\
\hline Proctotrupidae & & & & & & & 4.3 & & & & & & & פ.: & & \\
\hline Diapridae & & & & & & & & 4.0 & & & & & & & 18.2 & 6.7 \\
\hline Platygasterioae & & & & & & & 4.3 & 12.0 & & & & & & & & 20.0 \\
\hline \multicolumn{17}{|l|}{ Bethyloidea } \\
\hline Dryinidae & & & & & & & 4.3 & & & & & & & & & \\
\hline Formicidae & & & 8.3 & 2.2 & & & 87.0 & 40.0 & & 21.4 & 54.5 & 25.0 & & 18.2 & 81.8 & 53.3 \\
\hline \multicolumn{17}{|l|}{ Vespoidea } \\
\hline Yespidae & & & & & & & & 4.0 & & & & 8.3 & & & & 13.3 \\
\hline Pompilióae & & & & & & & 4.3 & & & & & & & & & \\
\hline Sprecidae & & & & & & & 4.3 & & & & & & & & & 20.0 \\
\hline \multicolumn{17}{|l|}{ Asodea } \\
\hline Halictuae & & & & & & & & & & & & & & & & 6.7 \\
\hline \multirow{2}{*}{\multicolumn{17}{|c|}{$\begin{array}{l}\text { Chorciata } \\
\text { Osteichthyes }\end{array}$}} \\
\hline & & & & & & & & & & & & & & & & \\
\hline Salmonitormes & & & & & & & & & & & & & & & & \\
\hline Salmonidae & & & & 2.2 & & & 8.7 & & & & & & & & & \\
\hline
\end{tabular}

Table continued on next page 
Percentage of Samples From Eastern Streams Containing Listed Taxa, by Season and Sample Type

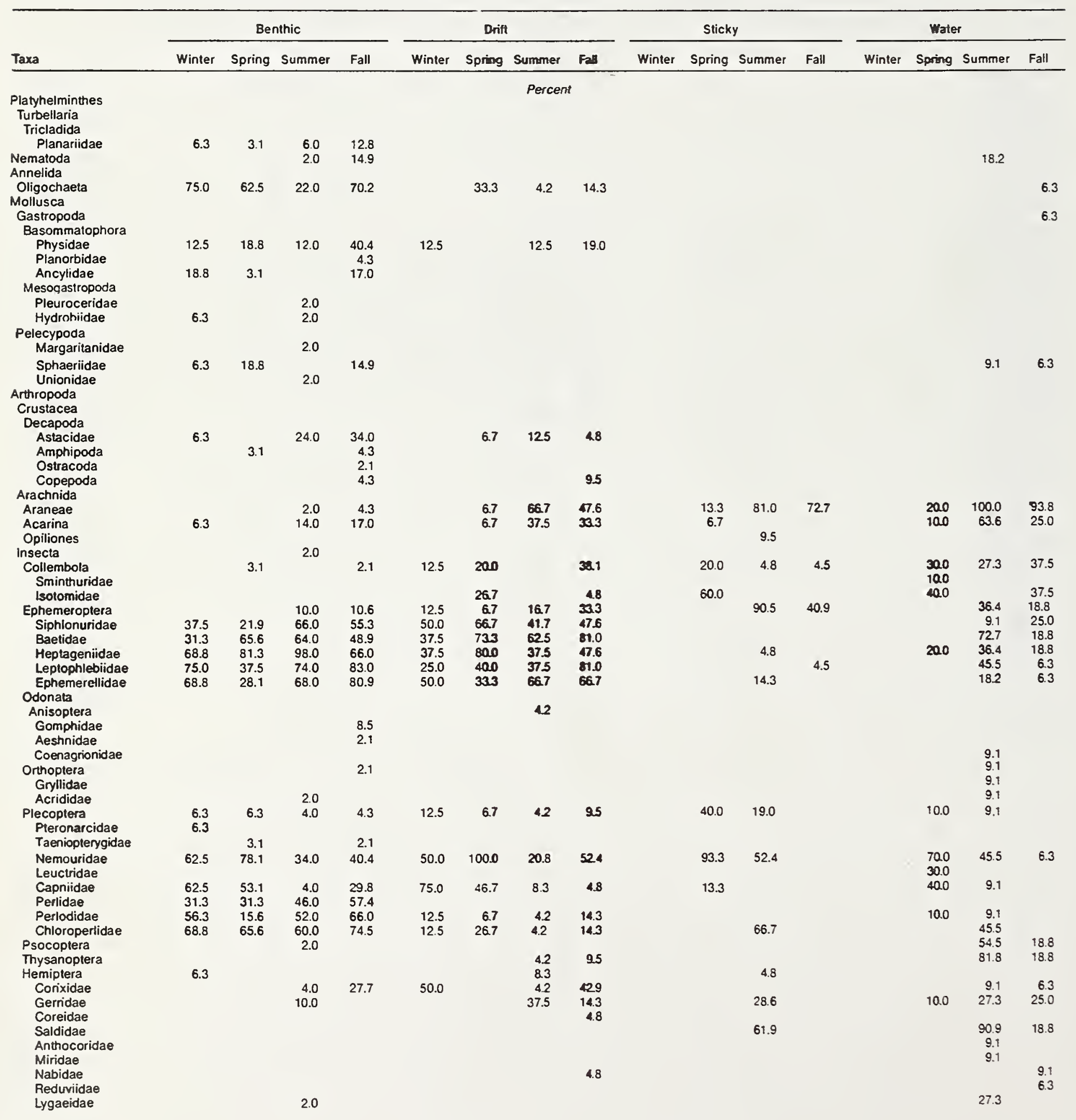

Table continued on next page. 


\begin{tabular}{|c|c|c|c|c|c|c|c|c|c|c|c|c|c|c|c|c|}
\hline \multirow[b]{2}{*}{ Taxa } & \multicolumn{4}{|c|}{ Benthic } & \multicolumn{4}{|c|}{ Dritt } & \multicolumn{4}{|c|}{ Sticky } & \multicolumn{4}{|c|}{ Water } \\
\hline & Winter & Spring & Summer & Fall & Winter & Spring & Summer & Fall & Winter & Spring & Summer & Fall & Winter & Spring & Summer & Fall \\
\hline \multicolumn{17}{|c|}{ Percent } \\
\hline Homoptera & & & & 2.1 & & & & 143 & & 13.3 & & & & 20.0 & 18.2 & 25.0 \\
\hline Cicadellidae & & & 16.0 & 25.5 & 12.5 & 13.3 & 25.0 & 619 & & 13.3 & 762 & 68.2 & & 30.0 & 90.9 & 68.8 \\
\hline $\begin{array}{l}\text { Cercopidae } \\
\text { Delphacidae }\end{array}$ & & & & & & & & & & & & & & 10.0 & $\begin{array}{l}27.3 \\
36.4\end{array}$ & 125 \\
\hline Psyllidae & & & & & & & & & & 13.3 & & & & 10.0 & & 18.8 \\
\hline $\begin{array}{l}\text { Aphididae } \\
\text { Eriosomatidae }\end{array}$ & & & 2.0 & & & 6.7 & 20.8 & $\begin{array}{r}71.4 \\
9.5\end{array}$ & & & 9.5 & 227 & & & 100.0 & $\begin{array}{l}68.8 \\
12.5\end{array}$ \\
\hline Coleoptera & & & 2.0 & & 12.5 & 13.3 & 8.3 & 190 & & 13.3 & 28.6 & 27.3 & & & 27.3 & 125 \\
\hline Noteridae & & & 2.0 & & & & & & & & & & & & & \\
\hline Carabidae & & & & & & & 4.2 & 9.5 & & & 9.5 & 9.1 & & & 45.5 & 6.3 \\
\hline Haliplidae & 6.3 & & 10.0 & 19.1 & & & 4.2 & 33.3 & & & & & & & & \\
\hline Dytiscidae & 25.0 & & 22.0 & 27.7 & 12.5 & 20.0 & 12.5 & 14.3 & & & & & & & 182 & \\
\hline Histerıdae & & & & & & & 4.2 & & & & & 4.5 & & & & \\
\hline Hydrophilidae & & 3.1 & 2.0 & & 12.5 & 20.0 & 16.7 & 42.9 & & 13.3 & 19.0 & 18.2 & & 10.0 & 54.5 & 18.8 \\
\hline $\begin{array}{l}\text { Hydraenidae } \\
\text { (Limnebiidae) }\end{array}$ & 6.3 & & & 2.1 & 50.0 & 26.7 & 125 & 476 & & & & & & & & 125 \\
\hline Melandryidae & 0.3 & & & 2.1 & 50.0 & 20.1 & 12.5 & 47.6 & & 6.7 & & & & & 9.1 & 12.5 \\
\hline Scarabaeidae & & & & & & & & & & & & & & & 182 & \\
\hline Ptiliidae & & & & & & & & 4.8 & & & & & & & 182 & 6.3 \\
\hline Chrysomelidae & & & & 2.1 & & & 4.2 & & & & & 4.5 & & & 273 & 18.8 \\
\hline Staphylinidae & & & & & 12.5 & 40.0 & 16.7 & 57.1 & & 13.3 & 14.3 & 54.5 & & 40.0 & 727 & 62.5 \\
\hline Scydmaenidae & & & & & & & 4.2 & & & & & & & & & \\
\hline Cantharidae & & & 2.0 & & & & & & & & 4.8 & & & & 27.3 & \\
\hline $\begin{array}{l}\text { Lamoyridae } \\
\text { Curculionıdae }\end{array}$ & & & & & & & & & & & & & & & 9.1 & \\
\hline (Nemonychidae) & & & & & & & & 4.8 & & & & & & & & \\
\hline Elateridae & & & & & & & 4.2 & & & & 4.8 & & & & 18.2 & \\
\hline Psephenidae & & & & 2.1 & & & & & & & & & & & & \\
\hline Elmidae & 43.8 & 37.5 & 98.0 & 87.2 & 25.0 & 6.7 & 54.2 & 71.4 & & & 4.8 & & & & 9.1 & \\
\hline $\begin{array}{l}\text { Scolytidae } \\
\text { Bostrichidae }\end{array}$ & & & & & & 6.7 & 4.2 & 4.8 & & & 4.8 & & & 10.0 & 182 & \\
\hline $\begin{array}{l}\text { Bostrichıdae } \\
\text { Nitidulidae }\end{array}$ & & & & & & & 8.3 & & & & & & & & & \\
\hline Nitidulidae & & & & & & & & & & & & & & & 18.2 & \\
\hline Coccinellidae & & & 2.0 & & & & 4.2 & 4.8 & & & & & & & 9.1 & \\
\hline Cerambycidae & & & & & & & & & & & & & & & 18.2 & \\
\hline Neuroptera & & & & & & & & & & & 4.8 & & & & & \\
\hline Sialidae & 12.5 & 3.1 & 10.0 & 55.3 & 12.5 & & & & & & & & & & & \\
\hline Chrysooidae & & & & & & & & & & & & & & & 9.1 & \\
\hline Trichootera & & & 2.0 & 8.5 & & 6.7 & 12.5 & 14.3 & & 6.7 & 81.0 & 45.5 & & & & \\
\hline Limneohilidae & 12.5 & 6.3 & 20.0 & 14.9 & 37.5 & 40.0 & 16.7 & 33.3 & & & 4.8 & & & & 18.2 & 25.0 \\
\hline Philopotamidae & 6.3 & & 12.0 & & & & & & & & & & & & & 31.3 \\
\hline Rhyacoohılidae & 12.5 & 43.8 & 12.0 & 8.5 & & 26.7 & & 4.8 & & & & & & & 54.5 & 6.3 \\
\hline Hydropsychidae & 18.8 & 12.5 & 18.0 & 27.7 & & & & 42.9 & & & & & & & 18.2 & \\
\hline Psychomylidae & & & 4.0 & 17.0 & & & & 4.8 & & & & & & & & \\
\hline Polycentrooodidae & & & & & & & & & & & & & & & 9.1 & \\
\hline Lepidostomatidae & 50.0 & 31.3 & 10.0 & 48.9 & 12.5 & 6.7 & & 38.1 & & & & & & & 27.3 & \\
\hline Glossosomatıdae & 12.5 & 6.3 & 12.0 & 8.5 & & & & 4.8 & & & & & & & & \\
\hline Hydroptulidae & 12.5 & & 4.0 & 17.0 & & & 4.2 & 4.8 & & & & & & & 54.5 & 25.0 \\
\hline Leotocerıdae & & & & & & & & & & & & & & & 9.1 & \\
\hline Lepidoptera & & & & & & 6.7 & & 4.8 & & & 38.1 & 136 & & & 27.3 & 6.3 \\
\hline $\begin{array}{l}\text { Satyridae } \\
\text { Hesoeridae }\end{array}$ & & & & & & & & & & & & & & & & 125 \\
\hline $\begin{array}{l}\text { Resperlldae } \\
\text { Noctuidae }\end{array}$ & & & & & & & & & & & & & & & & 18.8 \\
\hline $\begin{array}{l}\text { Noctuidae } \\
\text { Geometridae }\end{array}$ & & & & & & & & & & & & - & & & 182 & 6.3 \\
\hline Microlepidootera & & & & & & & 8.3 & & & & & & & & 54.5 & \\
\hline Pyralidae & & & & 170 & & 67 & & 95 & & & & & & & 727 & 6.3 \\
\hline Diptera & & 3.1 & 2.0 & & & $\begin{array}{r}6.8 \\
13.3\end{array}$ & 4.2 & 23.8 & & 200 & & & & & & \\
\hline Nematocera & & & & & & & & 9.5 & & $\begin{array}{r}20.0 \\
6.7\end{array}$ & 4.8 & 27.3 & & & 182 & 12.5 \\
\hline Tipulidae & 81.3 & 81.3 & 74.0 & 83.0 & 12.5 & & 8.3 & 57.1 & & 6.7 & & 45 & & & 9.1 & 6.3 \\
\hline Psychodidae & 25.0 & 25.0 & 4.0 & 36.2 & 12.5 & 67 & 8.3 & & & 6. 7 & $\begin{array}{r}76.2 \\
0.5\end{array}$ & 59.1 & & 10.0 & 90.9 & 93.8 \\
\hline Bleonariceridae & & 18.8 & & & & 6.7 & & 4.8 & & 33.3 & 9.5 & & & & 727 & 43.8 \\
\hline Dixidae & & & & & & 6.7 & 25.0 & 71.4 & & & & & & & 182 & 31.3 \\
\hline Ceratopogonidae & & 9.4 & 10.0 & 23.4 & & 13.3 & & 28.6 & & & 4.8 & 4.5 & & 90.9 & 43.8 & \\
\hline Chironomidae & 93.8 & 81.3 & 88.0 & 91.5 & 75.0 & 86.7 & 70.8 & 100.0 & & 73.3 & 85.7 & 86.4 & & 70.0 & 100.0 & 100.0 \\
\hline Simuliidae & 31.3 & 65.6 & 22.0 & 4.3 & & 80.0 & 37.5 & 28.6 & & 6.7 & 9.5 & 4.5 & & 10.0 & 9.1 & 25.0 \\
\hline Bibionidae & & & & & & & & 9.5 & & & & 4.5 & & & & 125 \\
\hline Mycetophilidae & & & & & & 13.3 & & 19.0 & & 20.0 & 47.6 & 68.2 & & 40.0 & 63.6 & 87.5 \\
\hline
\end{tabular}

Table continued on next page. 


\begin{tabular}{|c|c|c|c|c|c|c|c|c|c|c|c|c|c|c|c|c|}
\hline \multirow[b]{2}{*}{ Taxa } & \multicolumn{4}{|c|}{ Benthic } & \multicolumn{4}{|c|}{ Drift } & \multicolumn{4}{|c|}{ Sticky } & \multicolumn{4}{|c|}{ Water } \\
\hline & Winter & Spring & Summer & Fall & Winter & Spring & Summer & Fall & Winier & Spring & Summer & Fall & Winter & Spring & Summer & Fall \\
\hline \multicolumn{17}{|c|}{ Percent } \\
\hline $\begin{array}{l}\text { Sciaridae } \\
\text { Cedidiomyiidae }\end{array}$ & & 3.1 & 4.0 & 4.3 & & $\begin{array}{r}33.3 \\
6.7\end{array}$ & 20.8 & $\begin{array}{l}52.4 \\
14.3\end{array}$ & - & $\begin{array}{r}13.3 \\
6.7\end{array}$ & $\begin{array}{l}52.4 \\
19.0\end{array}$ & $\begin{array}{r}54.5 \\
9.1\end{array}$ & & & $\begin{array}{l}100.0 \\
100.0\end{array}$ & $\begin{array}{l}81.3 \\
43.8\end{array}$ \\
\hline Brachycera & & & & & & & & & & & & & & & & 6.3 \\
\hline Stratiomyidae & & 3.1 & & & & & & & & & & & & & & \\
\hline Tabanidae & 18.8 & 9.4 & 8.0 & 23.4 & & & & & & & 14.3 & & & & 9.1 & \\
\hline Rhagionidae & & & & & & & & & & & 14.3 & & & & 27.3 & \\
\hline Periscelididae & & & & & & & & & & & & & & & Q.1 & \\
\hline Asilidae & & & & & & & & & & & 61.9 & & & & 63.6 & \\
\hline Acroceridae & & & & & & & & & & & & & & & 27.3 & \\
\hline Bombyliidae & & & & & & & & & & & & & & & 9.1 & \\
\hline Empididae & & & 10.0 & 6.4 & & & 16.7 & 19.0 & & 20.0 & 61.9 & 100.0 & & 10.0 & 72.7 & 62.5 \\
\hline Dolichopodidae & & & 2.0 & & & & 12.5 & 14.3 & & & 95.2 & 50.0 & & 30.0 & 100.0 & 81.3 \\
\hline $\begin{array}{l}\text { Cyclorrhapha } \\
\text { Lauxaniidae }\end{array}$ & & & & & & & & & & & & & & & $\begin{array}{r}16.2 \\
91\end{array}$ & 12.5 \\
\hline Lonchopteridae & & & & & & & & & & & & & & & 9.1 & 6.3 \\
\hline Phoridae & & & & & & & & 33.3 & & & 14.3 & 4.5 & & 10.0 & 72.7 & $i 2.5$ \\
\hline Syrohidae & & & & & & & & & & & 28.6 & & & & 27.3 & \\
\hline Lonchaeidae & & & & & & & & & & & & & & 10.0 & & \\
\hline Sphaeroceridae & & & & & & & & & & 6.7 & & & & & 27.3 & \\
\hline Milichiidae & & & & & & & & & & & & & & & 27.3 & 63 \\
\hline Ephydridae & & & 2.0 & & & & 20.8 & 47.6 & & & 42.9 & $22 . \bar{T}$ & & 20.0 & 100.0 & 81.3 \\
\hline Drosophilidae & & & & & & & & & & & 4.8 & & & & 27.3 & 6.3 \\
\hline Chloropidae & & & & & & & & & & & & & & & 54.5 & \\
\hline Agromyzidae & & & & & & & & 9.5 & & & & & & & 36.4 & \\
\hline Clusiidae & & & & & & & & & & & & & & & 9.1 & \\
\hline Sciomyzıdae & & & & & & & & & & & & & & & 9.1 & \\
\hline Heleomyzidae & & & & & & & & & & & & & & & 18.2 & 12.5 \\
\hline Anthomyzıdae & & & & & & & & 4.8 & & 33.3 & & & & & 63.6 & 75.0 \\
\hline $\begin{array}{l}\text { Anthomylidae } \\
\text { (Scatophagidae) }\end{array}$ & & & & & & & & & & & & & & & 09 & \\
\hline Muscoidea & & & & & & & & 9.5 & & 13.3 & 95.2 & 95.5 & & & 9.1 & 6.3 \\
\hline Muscidae & & & & & & & & & & & & & & 10.0 & 72.7 & 31.3 \\
\hline \multicolumn{17}{|l|}{$\begin{array}{l}\text { Muscidae } \\
\text { Oestroidea }\end{array}$} \\
\hline Calliohorıdae & & & & & & & & & & & & & & & 27.3 & 25.0 \\
\hline Sarcophagıdae & & & & & & & & & & & & & & & 9.1 & \\
\hline Tachinıdae & & & & & & & & & & & & & & & 45.5 & 18.8 \\
\hline Hymenootera & & & & & & 6.7 & 12.5 & 14.3 & & & 23.8 & & & & 36.4 & \\
\hline \multicolumn{17}{|l|}{$\begin{array}{l}\text { Hymenootera } \\
\text { Apocrita }\end{array}$} \\
\hline Braconıdae & & & & 2.1 & & & 4.2 & 4.8 & & & & & & & 9.1 & \\
\hline Ichneumonidae & & & & 2.1 & & & 16.7 & 23.8 & & & 23.8 & 4.5 & & & 63.6 & 12.5 \\
\hline Chalcıdoıdea & & & & & & & 16.7 & 28.6 & & & 4.8 & 4.5 & & & 81.8 & 12.5 \\
\hline Mymaridae & & & 2.0 & & & & 4.2 & 14.3 & & & & & & & 18.2 & 12.5 \\
\hline Cynipoidea & & & & & 12.5 & & & 4.8 & & & & & & & 9.1 & 6.3 \\
\hline Cynıpidae & & & & & & & 4.2 & 4.8 & & & & & & & & \\
\hline Proctotrupoidea & & & & 2.1 & & & & 38.1 & & & 4.8 & & & & 45.5 & 25.0 \\
\hline Diapriidae & & & & & & & 4.2 & & & & & & & & & \\
\hline Platygasteridae & & & & & & & & 4.8 & & & & & & & & \\
\hline \multicolumn{17}{|l|}{ Bethyloidea } \\
\hline Formıcıdae & & & & & & 20.0 & 33.3 & 38.1 & & & 42.9 & & & 63.6 & 43.8 & \\
\hline Vespoidea & & & & & & & & & & & & & & & 27.3 & 63 \\
\hline Sphecidae & & & & & & & & & & & & & & & $\begin{array}{r}27.3 \\
9.1\end{array}$ & 6.3 \\
\hline Apoidea & & & & & & & & & & & & & & & 9.1 & 6.3 \\
\hline Halicıdae & & & & & & & & & & & & & & & 9.1 & \\
\hline Apidae & & & & & & & & & & & & & & & & 6.3 \\
\hline \multirow{2}{*}{\multicolumn{17}{|c|}{$\begin{array}{l}\text { Diplopoda } \\
\text { Chordata }\end{array}$}} \\
\hline & & & & & & & 8.3 & & & & & & & & & \\
\hline Cyoriniformes & & & & & & & & & & & & & & & & \\
\hline Catostomidae & & & 2.0 & 4.3 & & & 8.3 & & & & & & & & & \\
\hline Cyprinidae & & & & 2.1 & & & & & & & & & & & 91 & \\
\hline Coltidae & 6.3 & & 18.0 & 12.8 & & & 16.7 & 4.8 & & & & & & & 9.1 & \\
\hline Mammalia & & & & & & & & & & & & & & & & \\
\hline $\begin{array}{l}\text { Rodenti2 } \\
\text { Zapodidae }\end{array}$ & & & & & & & & & & & & & & & 9.1 & \\
\hline
\end{tabular}




\section{Appendix 3}

\section{Summary of Invertebrate Data For Each Season and Sample Type-Coastal Streams}

1. Composition

\begin{tabular}{|c|c|c|c|c|c|c|c|c|c|c|c|c|c|c|c|c|}
\hline \multirow[b]{2}{*}{ Taxa } & \multicolumn{4}{|c|}{ Benthic } & \multicolumn{4}{|c|}{ Dritt } & \multicolumn{4}{|c|}{ Sticky } & \multicolumn{4}{|c|}{ Water } \\
\hline & Winter & Spring & Summer & Fall & Winter & Spring & Summer & Fall & Winter & Spring & Summer & Fall & Winter & Spring & Summer & Fall \\
\hline & \multicolumn{16}{|c|}{ Percent } \\
\hline Oligochaeta & 0.5 & 0.2 & 0.2 & 0.6 & 0.6 & 0.1 & 0.1 & & & & & & 1.5 & 1.5 & & \\
\hline Gastropoda & .7 & 2.3 & 10.0 & 5.0 & 13.0 & 2.6 & 15.7 & 10.1 & 0.1 & & & & & & & \\
\hline Araneae & & & & & & 1 & .2 & 3 & .5 & 0.6 & & 1.2 & 1.5 & 1.9 & 3.6 & 3.4 \\
\hline Acarına & & & .1 & 1 & & .2 & 4 & 2 & & 1 & & 1 & 5 & 11 & .5 & .4 \\
\hline Collembola & & & & 1 & 6 & 4 & 2 & 2 & 3.7 & 13.5 & 04 & 3.0 & 5.0 & 132 & 1.0 & 9.7 \\
\hline Ephemeroptera & 65.9 & 59.3 & 20.5 & 17.4 & 24.5 & 68.3 & $41 . \overline{9}$ & 18.5 & 2.1 & 1.6 & 3 & 19 & 20 & $1 \overline{1}$ & 1.0 & 1.4 \\
\hline Orthoptera & & & & & & & & & & 6 & & 1 & & 1.0 & & .1 \\
\hline Plecoptera & 84 & 11.8 & 19.8 & 11.5 & 6.5 & 12.4 & $1 \mathrm{C} .3$ & 27.3 & 10.3 & 3.9 & 2 & 21 & 6.5 & 12 & 6 & .5 \\
\hline Psocoptera & & & & & & & .2 & & & & & & & & 2 & 1.9 \\
\hline Hemiptera & & & & & & 3 & 6 & .4 & & 13 & 7 & 1.0 & 3.0 & 3.4 & 41 & 1.7 \\
\hline Homoptera & & & .1 & & 3 & 1 & 3 & 7 & 2 & 1.4 & 4 & 2.0 & 1.5 & .1 & 5.7 & 4.8 \\
\hline Coleoptera & 1.9 & 13.6 & 27.3 & 32.3 & 4.7 & 3.1 & 6.8 & 2.8 & 3.3 & 10.0 & 3.9 & .9 & 3.5 & 3.4 & 6.1 & 1.6 \\
\hline Trichoptera & 7.0 & 5.7 & 10.2 & 10.3 & 23.9 & 5.5 & 6.0 & 244 & 2.8 & 5.4 & 9 & 94 & 2.5 & 45 & 2.6 & 3.0 \\
\hline Diptera & 15.4 & 7.0 & 11.1 & 21.9 & 24.8 & 6.7 & 16.1 & 143 & 76.8 & 60.3 & 92.9 & 75.6 & 72.0 & 645 & 72.6 & 68.2 \\
\hline Hymenoptera & & & & & .6 & 2 & .3 & 4 & .2 & 1.3 & 2 & 1.8 & & 1.9 & 1.4 & 2.9 \\
\hline Other 1 . & .2 & .1 & .7 & .8 & .5 & & .9 & .4 & & & .1 & .9 & .5 & 1.2 & .0 & .4 \\
\hline
\end{tabular}

y Other is the sum ol all taxa that did not comprise more than 1 percent of the lotal number of organisms within that season

2. Number of organisms and sample weights

\begin{tabular}{|c|c|c|c|c|c|c|c|c|c|c|c|c|c|c|c|c|}
\hline \multirow[b]{2}{*}{ Parameter } & \multicolumn{4}{|c|}{ Benthic } & \multicolumn{4}{|c|}{ Dritt } & \multicolumn{4}{|c|}{ Sticky } & \multicolumn{4}{|c|}{ Water } \\
\hline & Winter & Spring & Summer & Fall & Winter & Spring & Summer & Fall & Winter & Spring & Summer & Fall & Winter & Spring & Summer & Fall \\
\hline Number of samples & 24 & 31 & 43 & 47 & 11 & 15 & 30 & 19 & 16 & 15 & 24 & 23 & 2 & 9 & 14 & 17 \\
\hline $\begin{array}{l}\text { Mean number of } \\
\text { organisms per sample }\end{array}$ & 76.0 & 193.1 & 145.6 & 163.0 & 29.3 & 192.9 & 95.3 & 117.8 & 26.7 & 130.3 & 428.5 & 66.2 & 28.6 & 169.8 & 238.4 & 81.6 \\
\hline $\begin{array}{l}\text { Minimum number of } \\
\text { organisms per sample }\end{array}$ & 1 & 11 & 42 & 25 & 1 & 8 & 7 & 11 & 6 & 34 & 52 & 16 & 4 & 52 & 161 & 2 \\
\hline $\begin{array}{l}\text { Maximum number of } \\
\text { organisms per sample }\end{array}$ & 251 & 538 & 479 & 472 & 99 & 1689 & $39 i$ & 283 & 49 & 406 & 1694 & 145 & 72 & & & \\
\hline $\begin{array}{l}\text { Mean sample } \\
\text { weightig! }\end{array}$ & .050 & .274 & 498 & 210 & .103 & 233 & 501 & .263 & & & & & .025 & .206 & .136 & .079 \\
\hline $\begin{array}{l}\text { Minımum sample } \\
\text { weight(g) }\end{array}$ & .002 & .003 & .035 & .021 & .002 & .003 & .015 & .028 & & & & & .000 & .023 & .067 & .001 \\
\hline $\begin{array}{l}\text { Maximum sample } \\
\text { werght(g) }\end{array}$ & .270 & 1.064 & 1.811 & .812 & 601 & .962 & 1.923 & 1.223 & & & & & .087 & .417 & .264 & .362 \\
\hline
\end{tabular}




\section{Summary of Invertebrate Data for Each Season and Sample Type-Cascade Streams}

1. Composition

\begin{tabular}{|c|c|c|c|c|c|c|c|c|c|c|c|c|c|c|c|c|}
\hline \multirow[b]{2}{*}{ Taxa } & \multicolumn{4}{|c|}{ Benthic } & \multicolumn{4}{|c|}{ Drift } & \multicolumn{4}{|c|}{ Sticky } & \multicolumn{4}{|c|}{ Water } \\
\hline & Winter & Spring & Summer & Fall & Winter & Spring & Summer & Fall & Winter & Spring & Summer & Fall & Winter & Spring & Summer & Fall \\
\hline & \multicolumn{16}{|c|}{ Percent } \\
\hline Nematoda & 0.1 & 0.2 & 0.2 & 1.7 & & & & & & & & & & & 0.1 & \\
\hline Oligochaeta & .8 & 3.3 & 1.9 & 2.9 & 1.1 & 0.1 & 0.7 & 0.1 & & & & & & 0.1 & & \\
\hline Araneae & & & & & .1 & & .2 & & & & 0.3 & 0.1 & & .5 & 1.3 & 1.2 \\
\hline Acarina & & & .1 & & & 1 & 1.9 & .2 & & & & & & & .2 & .1 \\
\hline Collembola & .2 & & & .5 & .3 & .3 & .1 & 6.7 & 91.2 & 43.9 & 9.5 & 59.7 & & 255 & 23.9 & 33.0 \\
\hline Ephemeroptera & 50.1 & 60.5 & 47.2 & 25.5 & 34.7 & 62.5 & 45.0 & 22.7 & & .5 & 1.4 & .8 & & 1.6 & 1.2 & 1.1 \\
\hline Plecoptera & 33.5 & 19.6 & 17.7 & 28.4 & 30.9 & 10.0 & 4.3 & 23.9 & .6 & 7.5 & 1.1 & .2 & & 13.4 & 1.2 & 1.1 \\
\hline Psocoptera & & & & & & & & 2.0 & & & & & & & .3 & 1.4 \\
\hline Thysanoptera & & & & & & & & & & & & & & & 1.2 & \\
\hline Hemiptera & & & & & & & .2 & .1 & & & .6 & & & .1 & 1.8 & .3 \\
\hline Homoptera & & & & & & & 1.2 & 1.3 & & & 3 & .7 & & .5 & 1.7 & 2.1 \\
\hline Coleoptera & .4 & .6 & 3.4 & 3.4 & .9 & .5 & 4.8 & 4.5 & .1 & 1.0 & 1.5 & & & 2 & 3.7 & 1.7 \\
\hline Neuroptera & 3 & .1 & .4 & .4 & .1 & & & .1 & & & & & & & 1.1 & \\
\hline Trichoptera & 8.4 & 3.7 & 5.7 & 8.6 & 18.6 & 4.1 & 4.8 & 5.4 & .2 & .7 & 5.5 & 1.3 & & .1 & 3.7 & 1.9 \\
\hline Diptera & 4.5 & 11.4 & 22.3 & 26.8 & 12.9 & 22.0 & 35.4 & 30.9 & 7.9 & 46.4 & 78.9 & 35.0 & & 57.4 & 57.1 & 53.3 \\
\hline Hymenoptera & & & & & & & 1.1 & 1.6 & & & .4 & .5 & & 4 & 1.1 & 2.3 \\
\hline Other's & 1.7 & .6 & 1.1 & 1.8 & .4 & .4 & .3 & .5 & & & .5 & 1.7 & & .2 & .4 & .5 \\
\hline
\end{tabular}

y Other is the sum of all taxa that did not comprise more than 1 percent of the total number of organisms within that season.

2. Number of organisms and sample weights

\begin{tabular}{|c|c|c|c|c|c|c|c|c|c|c|c|c|c|c|c|c|}
\hline \multirow[b]{2}{*}{ Parameter } & \multicolumn{4}{|c|}{ Benthic } & \multicolumn{4}{|c|}{ Dritt } & \multicolumn{4}{|c|}{ Sticky } & \multicolumn{4}{|c|}{ Water } \\
\hline & Winter & Spring & Summer & Fall & Winter & Spring & Summer & Fall & Winter & Spring & Summer & Fall & Winter & Spring & Summer & Fall \\
\hline Number of samples & 16 & 29 & 35 & 48 & 12 & 13 & 26 & 21 & 8 & 16 & 24 & 22 & & 15 & 16 & 11 \\
\hline $\begin{array}{l}\text { Mean number of } \\
\text { organisms per sample }\end{array}$ & 1243 & 227.6 & 144.2 & 132.2 & 177.0 & 576.5 & 170.4 & 85.1 & 77.4 & 46.4 & 331.8 & 129.6 & & 54.7 & 594.8 & 131.7 \\
\hline $\begin{array}{l}\text { Minimum number of } \\
\text { organisms per sample }\end{array}$ & 28 & 41 & 30 & 19 & 75 & 75 & 1 & 10 & 4 & 5 & 66 & 13 & & 4 & 22 & 45 \\
\hline $\begin{array}{l}\text { Maximum number of } \\
\text { organisms per sample }\end{array}$ & 238 & 657 & 348 & 396 & 318 & 1445 & 535 & 251 & 424 & 305 & 2725 & 617 & & 130 & 3182 & 329 \\
\hline $\begin{array}{l}\text { Mean sample } \\
\text { weight(g) }\end{array}$ & .058 & .171 & .141 & .068 & .063 & .240 & .090 & .057 & & & & & & .166 & .673 & .093 \\
\hline $\begin{array}{l}\text { Minimum sample } \\
\text { weight(g) }\end{array}$ & .004 & .040 & .021 & .005 & .012 & .041 & .001 & .004 & & & & & & .001 & .091 & .001 \\
\hline $\begin{array}{l}\text { Maximum sample } \\
\text { weight(g) }\end{array}$ & .210 & .537 & .587 & .318 & .147 & .575 & .404 & .639 & & & & & & 1.180 & 2.788 & .279 \\
\hline
\end{tabular}




\section{Summary of Invertebrate Data for Each Season and Sample Type-Central Streams}

1. Composition

\begin{tabular}{|c|c|c|c|c|c|c|c|c|c|c|c|c|c|c|c|c|}
\hline \multirow[b]{2}{*}{ Taxa } & \multicolumn{4}{|c|}{ Benthic } & \multicolumn{4}{|c|}{ Drift } & \multicolumn{4}{|c|}{ Sticky } & \multicolumn{4}{|c|}{ Water } \\
\hline & Winter & Spring & Summer & Fall & Winter & Spring & Summer & Fall & Winter & Spring & Summer & Fall & Winter & Spring & Summer & Fall \\
\hline & \multicolumn{16}{|c|}{ Percent } \\
\hline Tricladida & 0.3 & 0.1 & 3.3 & 2.0 & 0.1 & 0.1 & & 0.1 & & & & & & & & \\
\hline Oligochaeta & 1.0 & 1.7 & 1.6 & 1.3 & .3 & .2 & & .1 & & & & & & & & \\
\hline Araneae & & & & & & .1 & 0.4 & .2 & & 0.1 & 0.4 & 0.5 & & 0.2 & 2.9 & 1.1 \\
\hline Collembola & & & & & & .1 & .1 & .2 & & 87.5 & 44.0 & 46.8 & & 95.5 & .9 & 16.7 \\
\hline Ephemeroptera & 31.7 & 23.0 & 33.5 & 34.3 & 33.6 & 31.8 & 39.6 & 31.7 & & .5 & .6 & 1.1 & & .1 & .4 & 1.1 \\
\hline Plecoptera & 28.1 & 20.4 & 11.1 & 11.9 & 31.0 & 37.6 & .9 & 12.3 & & 2.7 & .2 & .2 & & .8 & .4 & .6 \\
\hline Thysanoptera & & & & & & & & & & & & & & & 3.8 & .2 \\
\hline Hemiptera & & & .1 & & .1 & & 1.0 & .2 & & & .3 & & & & 1.3 & .3 \\
\hline Homoptera & & & .7 & .1 & & & 8.8 & 8.5 & & .8 & 4.4 & 8.7 & & .2 & 12.7 & 5.0 \\
\hline Coleoptera & 4.3 & 2.7 & 12.9 & 9.6 & .4 & .3 & 12.5 & 14.7 & & .2 & .4 & 1.7 & & .8 & 13.2 & 1.4 \\
\hline Trichoptera & 6.9 & 10.4 & 16.7 & 9.3 & 1.6 & 3.9 & 3.2 & 7.0 & & .2 & 2.2 & 11.1 & & .1 & 5.4 & 10.6 \\
\hline Diptera & 27.6 & 41.5 & 19.7 & 31.1 & 32.9 & 25.8 & 26.3 & 23.0 & & 7.7 & 46.9 & 29.1 & & 2.2 & 55.7 & 61.0 \\
\hline Hymenoptera & & & .1 & & & & 6.5 & 1.5 & & .2 & .6 & 6 & & .1 & 2.1 & 1.6 \\
\hline Other 1 & .1 & .2 & .5 & .4 & & .1 & .7 & .5 & & .1 & & .2 & & & 1.2 & 4 \\
\hline
\end{tabular}

Y Other is the sum of all taxa that did not comprise more than 1 percent of the total number of of ganisms within that season.

2. Number of organisms and sample weights

\begin{tabular}{|c|c|c|c|c|c|c|c|c|c|c|c|c|c|c|c|c|}
\hline \multirow[b]{2}{*}{ Parameter } & \multicolumn{4}{|c|}{ Benthic } & \multicolumn{4}{|c|}{ Dritt } & \multicolumn{4}{|c|}{ Sticky } & \multicolumn{4}{|c|}{ Water } \\
\hline & Winter & Spring & Summer & Fall & Winter & Spring & Summer & Fall & Winter & Spring & Summer & Fall & Winter & Spring & Summer & Fall \\
\hline $\begin{array}{l}\text { Number of samples } \\
\text { Mean number of } \\
\text { organisms per sample }\end{array}$ & $\begin{array}{c}16 \\
284.3\end{array}$ & $\begin{array}{l}30 \\
330.5\end{array}$ & $\begin{array}{c}48 \\
153.0\end{array}$ & $\begin{array}{c}45 \\
302.1\end{array}$ & $\begin{array}{c}8 \\
235.9\end{array}$ & $\begin{array}{c}14 \\
237.5\end{array}$ & $\begin{array}{c}21 \\
243.2\end{array}$ & $\begin{array}{c}21 \\
340.8\end{array}$ & & $\begin{array}{c}14 \\
380.7\end{array}$ & $\begin{array}{c}22 \\
930.7\end{array}$ & $\begin{array}{c}24 \\
223.5\end{array}$ & 11 & $\begin{array}{c}11 \\
892.5\end{array}$ & $\begin{array}{c}15 \\
376.9\end{array}$ & 222.9 \\
\hline $\begin{array}{l}\text { Minimum number of } \\
\text { organisms per sample }\end{array}$ & 39 & 3 & 23 & 1 & 29 & 10 & 8 & 10 & & 21 & 43 & 38 & 5 & 5 & 56 & \\
\hline $\begin{array}{l}\text { Maximum number of } \\
\text { organisms per sample }\end{array}$ & 909 & 1054 & 628 & 765 & 773 & 888 & 1224 & 1606 & & 2033 & 11063 & 2378 & & 5654 & 1681 & 459 \\
\hline $\begin{array}{l}\text { Mean sample } \\
\text { weight }(g)\end{array}$ & .10 & 20 & .160 & .138 & .09 & & .096 & .085 & & & & & .09 & & 01 & \\
\hline $\begin{array}{l}\text { Minimum sample } \\
\text { weight(g) }\end{array}$ & .00 & .00 & .005 & .000 & .016 & & .003 & .001 & & & & & .03 & & 26 & \\
\hline $\begin{array}{l}\text { Maximum sample } \\
\text { weight(g) }\end{array}$ & .218 & .50 & 1.348 & .653 & .21 & & .581 & .655 & & & & & .13 & .73 & 32 & \\
\hline
\end{tabular}




\section{Summary of Invertebrate Data for Each Season and Sample Type-Eastern Streams}

1. Cumposition

\begin{tabular}{|c|c|c|c|c|c|c|c|c|c|c|c|c|c|c|c|c|}
\hline \multirow[b]{2}{*}{ Taxa } & \multicolumn{4}{|c|}{ Benthic } & \multicolumn{4}{|c|}{ Drift } & \multicolumn{4}{|c|}{ Slicky } & \multicolumn{4}{|c|}{ Water } \\
\hline & Winter & Spring & Surnmer & Fall & Winter & Spring & Summer & Fall & Winter & Spring & Summer & Fall & Winter & Spring & Summer & Fall \\
\hline & \multicolumn{16}{|c|}{ Percent } \\
\hline Oligochaeta & 2.3 & 3.6 & 0.3 & 40 & 0.2 & 0.4 & 0.4 & & & & & & & & & \\
\hline Gastropoda & .9 & .2 & .4 & 4.4 & 1.8 & & .3 & 0.1 & & & & & & & & \\
\hline Decapoda & 1.0 & & .4 & .5 & & & .3 & .1 & & & & & & & & \\
\hline Araneae & & & & & & & 1.6 & .4 & & 0.8 & 1.1 & 1.8 & & 0.6 & 2.6 & 3.3 \\
\hline Acarina & .1 & & .2 & .1 & & & 1.7 & 1.4 & & .2 & & & & .6 & .4 & .2 \\
\hline Collembola & & & & & .6 & .4 & & 1.0 & & 53.5 & & & & 33.6 & .1 & 7.1 \\
\hline Ephemeroptera & 31.1 & 9.8 & 41.4 & 30.6 & 33.2 & 5.4 & 18.8 & 11.7 & & & 7.1 & 2.5 & & .6 & 2.0 & 1.8 \\
\hline Plecoptera & 20.9 & 31.7 & 6.9 & 9.3 & 41.0 & 76.0 & 2.4 & 1.6 & & 15.3 & 6.0 & & & 40.3 & 3.2 & \\
\hline Hemiptera & .1 & & .2 & 1.1 & 2.7 & & 4.3 & .3 & & & .7 & & & .9 & 1.2 & .6 \\
\hline Homoptera & & & .3 & .8 & .3 & .1 & 2.4 & 52.8 & & 1.2 & 8.5 & 36.7 & & 3.5 & 6.2 & 7.1 \\
\hline $\begin{array}{l}\text { Coleoptera } \\
\text { Neuroptera }\end{array}$ & $\begin{array}{r}8.1 \\
7\end{array}$ & 1.6 & 17.5 & $\begin{array}{l}8.8 \\
1.4\end{array}$ & $\begin{array}{r}3.3 \\
3\end{array}$ & 1.3 & 5.1 & 3.9 & & 1.2 & .7 & 2.5 & & 5.7 & 1.5 & 3.0 \\
\hline Trichoptera & 8.2 & 2.8 & 1.7 & 12.0 & 1.2 & .6 & 1.2 & 1.0 & & .2 & 2.5 & 1.9 & & & .9 & 1.7 \\
\hline Lepidoptera & & & & 3 & & .1 & 23.3 & 1.0 & & & .3 & .1 & & & 1.4 & .5 \\
\hline Diptera & 26.4 & 49.9 & 29.7 & 26.4 & 15.1 & 15.5 & 34.5 & 24.2 & & 26.9 & 72.0 & 54.4 & & 13.5 & 77.2 & 72.2 \\
\hline Hymenoptera & & & & & .3 & .1 & 2.5 & 1.1 & & & 1.0 & .1 & & & 1.8 & 1.9 \\
\hline Other', & .2 & .4 & .6 & .3 & & .1 & 1.2 & .4 & & .7 & .1 & & & .7 & 1.5 & .6 \\
\hline
\end{tabular}

1 Other is the sum of all taxa that did nol comprise more than 1 percent of the total number of organisms within that season.

2. Number of organisms and sample weights

\begin{tabular}{|c|c|c|c|c|c|c|c|c|c|c|c|c|c|c|c|c|}
\hline \multirow[b]{2}{*}{ Parameter } & \multicolumn{4}{|c|}{ Benthic } & \multicolumn{4}{|c|}{ Drift } & \multicolumn{4}{|c|}{ Sticky } & \multicolumn{4}{|c|}{ Water } \\
\hline & Winter & Spring & Summer & Fall & Winter & Spring & Summer & Fall & Winter & Spring & Summer & Fall & Winter & Spring & Summer & Fall \\
\hline Number of samples & 16 & 32 & 50 & 47 & 8 & 14 & 22 & 21 & & 15 & 21 & 22 & & 10 & 11 & 16 \\
\hline $\begin{array}{l}\text { Mean number of } \\
\text { organisms per sample }\end{array}$ & 91.6 & 121.4 & 137.9 & 278.6 & 42.1 & 221.7 & 63.8 & 393.9 & & 40.1 & 280.7 & 98.1 & & 80.3 & 653.6 & 138.3 \\
\hline $\begin{array}{l}\text { Minimum number of } \\
\text { organisms per sample }\end{array}$ & 6 & 1 & 1 & 12 & 6 & 11 & 5 & 2 & & 5 & 18 & 17 & & 1 & 130 & 5 \\
\hline $\begin{array}{l}\text { Maximum number of } \\
\text { organisms per sample }\end{array}$ & 356 & 459 & 526 & 1174 & 120 & 1125 & 501 & 3859 & & 152 & 979 & 282 & & 513 & 1770 & 336 \\
\hline $\begin{array}{l}\text { Mean sample } \\
\text { weight }(g)\end{array}$ & .264 & .052 & .106 & .355 & .043 & .043 & .300 & .074 & & & & & & .026 & .299 & .076 \\
\hline $\begin{array}{l}\text { Minimum sample } \\
\text { weight(g) }\end{array}$ & .003 & .000 & .001 & .012 & .005 & .004 & .000 & .003 & & & & & & .001 & .021 & .004 \\
\hline Maximum sample & 2.697 & .248 & 1.121 & 2.318 & .180 & .185 & 3.525 & .503 & & & & & & .067 & .720 & .166 \\
\hline
\end{tabular}




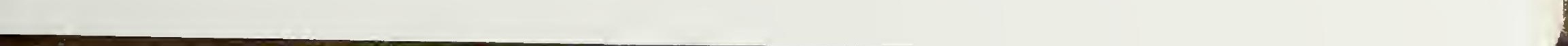



Porler, Pamela E.; Meehan, William R. Seasonal species composition of invertebrates in several Oregon streams. Res. Pap. PNW-RP-382. Portland, OR: U.S. Department of Agriculture, Forest Service, Pacific Northwest Research Station; 1987. 36 p.

The invertebrate communities of eight Oregon streams were sampled seasonally from 197410 1976. Benthic, drift, and iwo types of aerial-trap samples were collected. Occurrence and percentage composition are summarized by sample type, season, and geographic area (coastal, Cascade, central, and eastern Oregon). Within 276 families, 426 taxa were identified; the 20 families most prevalent within each sample type accounted for the majority of organisms collected (77.8-91.8 percent). In most areas and seasons, Diptera and Ephemeroptera logether comprised over half of the invertebrates collected.

Keywords: Invertebrata, aquatic life, Oregon

The Forest Service of the U.S. Department of Agriculture is dedicated to the principle of multiple use management of the Nation's forest resources for sustained yields of wood, water, forage, wildlife, and recreation. Through forestry research, cooperation with the States and private forest owners, and management of the National Forests and National Grasslands, it strives - as directed by Congress - to provide increasingly greater service to a growing Nation.

The U.S. Department of Agriculture is an Equal Opportunity Employer. Applicants for all Department programs will be given equal consideration without regard to age, race, color, sex, religion, or national origin.

Pacific Northwest Research Station 319 S.W. Pine St

P.O. Box 3890

Portland, Oregon 97208 
U.S. Department of Agriculture

BULK RATE POSTAGE -

319 S.W. Pine Street

P.O. Box 3890

USDA-FS

Portland, Oregon 97208

Official Business

Penalty for Private Use, $\$ 300$ 